\title{
Guidelines for the Management of Diabetic Macular Edema by the European Society of Retina Specialists (EURETINA)
}

\author{
Ursula Schmidt-Erfurth ${ }^{a}$ Jose Garcia-Arumi ${ }^{b}$ Francesco Bandello ${ }^{c}$

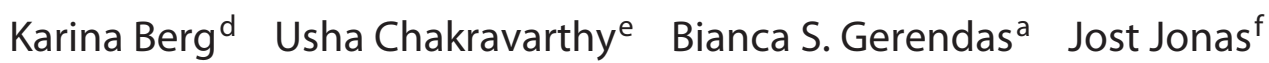 \\ Michael Larsen ${ }^{g}$ Ramin Tadayoni ${ }^{\text {h }}$ Anat Loewenstein ${ }^{i}$

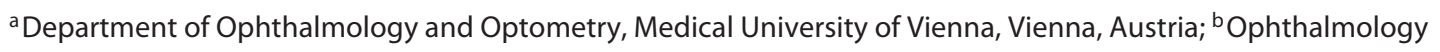 \\ Department, Universidad Autónoma de Barcelona, Barcelona, Spain; ${ }^{\circ}$ Department of Ophthalmology, Ospedale San

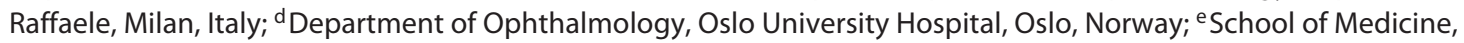 \\ Dentistry, and Biomedical Sciences, The Queen's University of Belfast, Belfast, UK; ' Department of Ophthalmology, \\ Medical Faculty Mannheim of the Ruprecht-Karls University Heidelberg, Mannheim, Germany; ${ }^{9}$ Faculty of Health \\ and Medical Sciences, University of Copenhagen, Copenhagen, Denmark; ${ }^{h}$ Department of Ophthalmology, Hôpital \\ Lariboisière, AP-HP, Université Paris 7 - Sorbonne Paris Cité, Paris, France; 'Department of Ophthalmology, Tel Aviv \\ Medical Center, Tel Aviv University, Tel Aviv, Israel
}

\section{Keywords}

Diabetic macular edema · Anti-VEGF therapy · Steroids

\begin{abstract}
Diabetic retinal disease is envisioned to become the plague of the coming decades with a steep increase of worldwide diabetes incidence followed by a substantial rise in retinal disease. Improvements in diagnostic and therapeutic care have to cope with this dilemma in a clinically and socioeconomically efficient manner. Laser treatment has found a less destructive competitor in pharmacological treatments. As a consequence of recent rigorous clinical trials, laser photocoagulation is no longer recommended for the treatment of diabetic macular edema (DME), and anti-vascular endothelial growth factor therapy has emerged as first-line therapy. Steroids have maintained a role in the management of chronically persistent DME. The paradigm shifts in therapy are accompanied by a substantial break-through in diagnos-
\end{abstract}

\section{KARGER}

E-Mail karger@karger.com

www.karger.com/oph tics. The following guidance for the management of DME has been composed from the best updated knowledge of leading experts in Europe and represents another volume in the series of EURETINA recommendations for the management of retinal disease.

(c) 2017 S. Karger AG, Basel

\section{Introduction}

The prevalence of diabetic macular edema (DME) is continuously rising worldwide and has become one of the major causes of vision loss in the working-age population. Clinical parameters and new diagnostic parameters from imaging with optical coherence tomography (OCT) as well as the overall advances of OCT technology have been identified to stage the disease. However, a large variety of therapeutic strategies are available to the ophthalmologist: laser photocoagulation, anti-vascular endothe- 


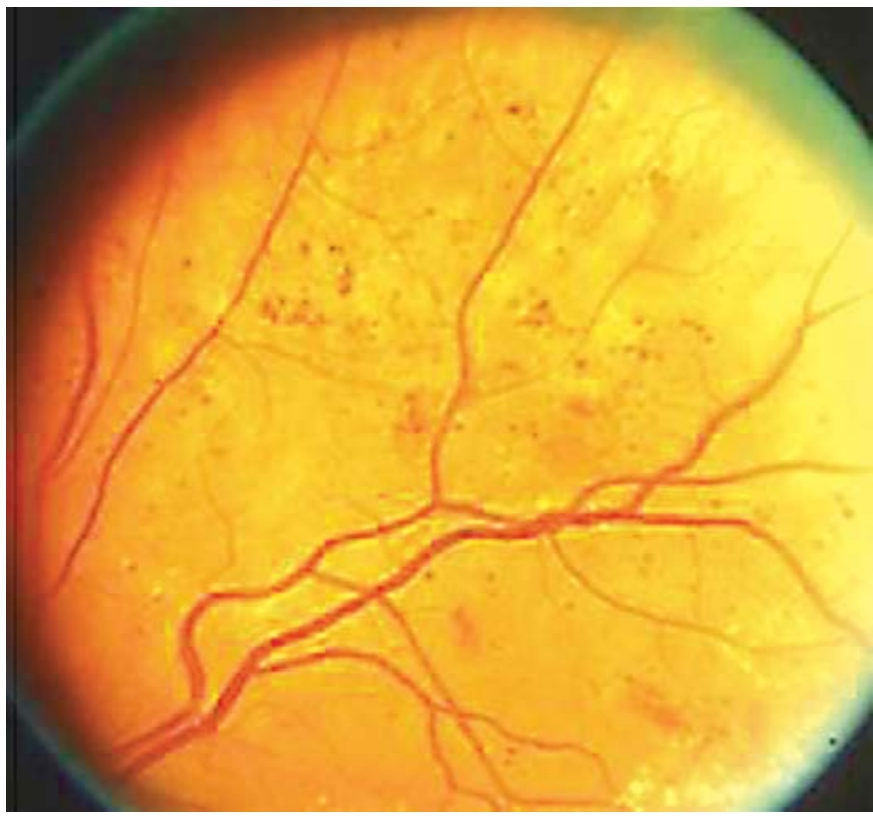

Fig. 1. Standard photograph depicting MA and hemorrhages from diabetic retinopathy study. Report number 6 and 7. Courtesy of Investigative Ophthalmology \& Visual Science [166].

lial growth factor (VEGF), steroid and surgical therapy are applied with different procedures and their own complications. A novel era of DME therapy has started with these diverse approaches. These guidelines shall give an overview on the current available diagnostic and therapeutic procedures and recommend their application.

\section{Clinical Features of Diabetic Retinopathy}

Diabetic retinopathy (DR) is the term applied to describe the microvascular abnormalities that are seen in the fundus of persons with diabetes on clinical examination or on color fundus photography. The earliest and the least severe manifestation is the dot-like microaneurysms (MA) which are localized saccular outpouchings of the capillary wall and appear as tiny red dots with sharp margins. MA occur frequently in relation to areas of capillary nonperfusion, but the latter is an angiographic diagnosis. Retinal hemorrhages are another important manifestation of DR and are found throughout the fundus (Fig. 1) They may be flame-shaped, if located in the nerve fiber layer, or dot or blot-like, if located in the middle layers of the retina. Intraretinal microvascular abnormalities (IRMA) are tortuous and dilated intraretinal microvascu-

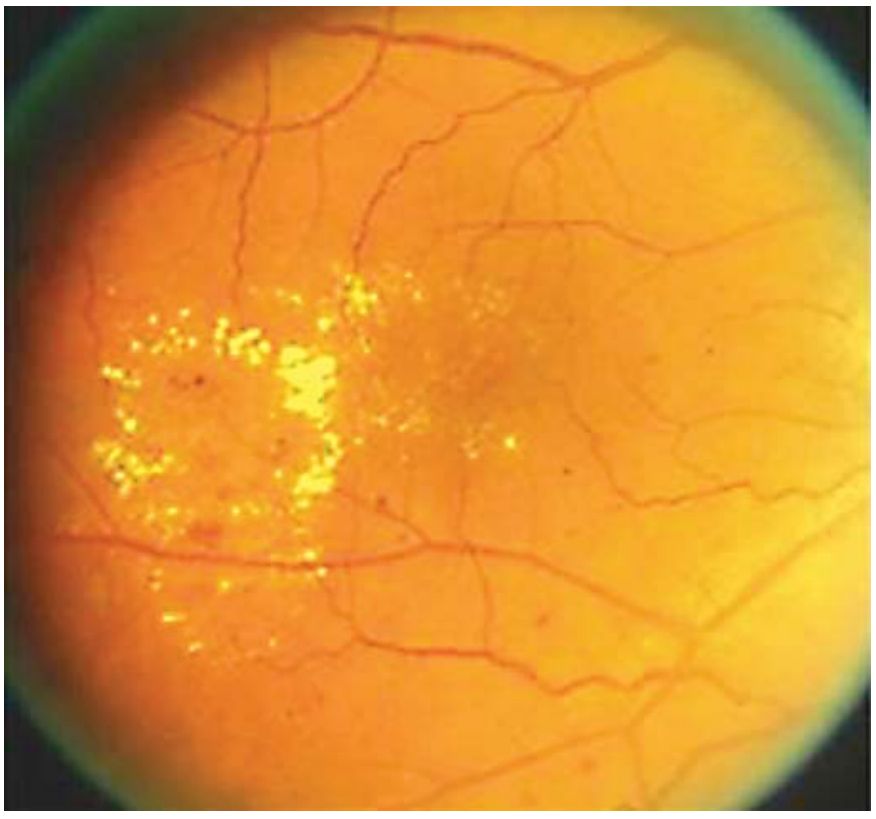

Fig. 2. Severe lipid exudation with hard exudates from grouped MA from diabetic retinopathy study. Report number 6 and 7 . Courtesy of Investigative Ophthalmology \& Visual Science [166].

lar segments. Venous dilation and beading are typical DR-related changes, and the latter in particular represent focal increases in venous caliber, resembling a string of beads. Hard exudates are another manifestation of DR, arising as a consequence of chronic localized leakage from the retinal vessels (Fig. 2). Hard exudates are mainly composed of lipid and appear as yellowish white lesions usually with distinct margins and occur close to clusters of MAs. Soft exudates, otherwise termed cotton wool spots, arise when there is a block in the flow of axoplasm in the retinal nerve fiber layer which occurs due to focal ischemia. They represent infarcted inner retina and appear as greyish-white round or oval areas with ill-defined feathery edges.

\section{Diabetic Macular Edema}

DME represents an accumulation of fluid within the central portion of the retina, which arises as a consequence of failure of the blood-retinal barrier (BRB). Diffuse edema is caused by extensive capillary leakage, whereas localized edema is caused by focal leakage from grouped MAs. DME can occur in isolation without other signs of microangiopathy in the fundus; therefore, it merits being classified as a separate entity. It is often associated with hard exudates and causes blurring and distortion of central vi- 
sion, which is reflected in a reduction in best-corrected visual acuity (BCVA). The Wisconsin Epidemiologic Study of Diabetic Retinopathy found that $20 \%$ of patients with type 1 diabetes and $25 \%$ of those with type 2 diabetes will develop DME after 10 years of follow-up [1].

\section{Proliferative DR}

This entity is described in the context of the management of DME as diagnostic and therapeutic studies have recently shown that the pathways of DME and DR are tightly interconnected and that pharmacological therapy affects both manifestations in an interchangeable manner.

Proliferative DR (PDR) refers to the development of vascular and fibrous tufts that arise from the retinal blood vessels and ramify into a network that spreads either within the retina or at the interface between the retina and the vitreous. Retinal hypoxia is the main driver for neovascularization, and as these vessels carry little or no pericytes, they are friable and bleed easily. The neovascularization seen on the fundus has been described as new vessels elsewhere (NVE) when located away from the optic disc, and as new vessels on the disc (NVD) when either on the optic disc or within 1 disc diameter of its margin. Hemorrhage from these vessels can lie within the retina, at the vitreoretinal interface or in severe cases within the vitreous. When preretinal, the hemorrhage may be crescentic shaped, oval, or linear. Hemorrhage further forward into the vitreous cavity is considered vitreous hemorrhage. The presence of blood particularly in the macular retina and within the vitreous leads to severe loss of vision. Blood is also inimical to the integrity of the retina. Subsequent development of fibrous bands resulting from organized hemorrhage can consecutively result in traction of the retina.

\section{Angiographic Manifestations in DME}

\section{Rationale}

Fluorescein angiography (FA) has been a valuable diagnostic tool in DR for decades and is formally recognized as an essential component in the assessment of severity of the pathology and delineation of location of retinal alteration to allow appropriate and targeted laser therapy [2]. However, there is currently only moderate consensus on the role of FA in the management of DME, as nowadays some clinicians would base treatment decision on the basis of OCT only. Nonetheless, FA remains the only commonly approved modality that can distin-

Guidelines for the Management of Diabetic Macular Edema by EURETINA guish nonleaking from leaking MA, define clearly the presence of IRMA, and delineate areas of capillary nonperfusion and widening of the foveal avascular zone (FAZ) in the macular retina [3]. Thus, an obvious indication for FA in relation to DME is to have accurate information on the location of areas to eventually be treated by focal laser. With the advent of anti-VEGF therapies with the corresponding reduction in the need for laser, and the detailed morphological imaging possibilities with highresolution OCT and the newest modality of OCT angiography (OCT-A), questions have arisen on the need for conventional FA. However, standard spectral-domain OCT (SD-OCT) cannot identify foveal ischemia and widening of the FAZ which are important prognostic indicators of outcome. First studies with OCT-A suggest that this modality will be able to quantify the aforementioned [4-10]. A major advantage of OCT-A versus FA is the potential to focus on different retinal layers in depths and highlight alterations at the level of the deep capillary plexus as a primary event in DR. However, MA often remain silent in the flow-based OCT-A modality. To date, FA allows a more comprehensive assessment of the extent of the morphological damage to the macular microcirculation prior to initiation of treatment and therefore better monitoring of change and responsiveness to treatment. This might change in the near future as many studies are currently conducted on the interpretation of OCT-A and as this technology advances fast. Nevertheless, as OCT-A reproduces perfusion rather than structural vascular features, the OCT-A technology in terms of hardware, e.g. swept source, longer wavelength, and software, e.g. improved algorithms for slow flow detection and elimination of projection artifacts, is subject to intensive scientific evaluation not resulting in solid clinical conclusions yet. Another important attribute of FA is the ability to scrutinize the retinal periphery with the recent advances in widefield imaging.

Widefield angiography is helpful for visualizing areas of peripheral neovascularization and assessment of the perfusion status of the peripheral retina. There is increasing evidence of peripheral nonperfusion in eyes with DME. These nonperfused peripheral regions act as the source of growth factors such as VEGF or inflammatory cytokines, which in turn create exudative pathology within the macula. Targeted ablation of areas of peripheral ischemia could switch off growth factor release and improve central retinal morphology and function. The identification and monitoring of these areas of peripheral ischemia during treatment with anti-VEGF agents could be used as a sensor or biomarker for therapeutic effect in the future.

Ophthalmologica 2017;237:185-222

DOI: $10.1159 / 000458539$ 


\section{Evidence}

In DME, the appearance of the macula on FA is highly characteristic, with MA and IRMA interspersed between areas of focal or diffuse hyperfluorescence representing leakage from the incompetent macular microcirculation [11]. In addition, regions of capillary loss and dilation, arteriolar abnormalities and cystoid changes can be observed. When DME leakage is focal from groups of MA, there is usually surrounding accumulation of lipid that assumes a circinate distribution [3]. Ectatic dilated leaking perifoveal capillaries are seen and can be difficult to distinguish from other conditions such as perifoveal telangiectasia [12], thus emphasizing the importance of obtaining an FA prior to DME therapy initiation. The Early Treatment Diabetic Retinopathy Study (ETDRS) relied on the use of FA as a guide for focal treatment of individual leaking MA and macular grid laser to areas of diffuse leakage and capillary nonperfusion with therapeutic benefit [11]. Furthermore, in one study when using ablative therapies such as laser, the use of FA to delineate the areas requiring treatment resulted in improved accuracy [2]. More recently, the relationships between OCT and FA findings have been explored and the various characteristics related to each other indicating that both overlap and the complementarity of findings $[13,14]$. Simultaneous FA and SD-OCT allow improved characterization of DME features and allow distinction of perfused MA from nonperfused MA as well as the ability to localize fluid to different retinal layers [15]. In a recent study, abnormalities of choroidal perfusion were detected by indocyanine green angiography, and the combination of this imaging modality along with enhanced-depth OCT imaging and FA were reported as superior indicators of ocular perfusion status in diabetic eyes [16]. Emerging data also indicate that regular OCT cannot replace FA as it is not possible to predict FAZ outline and size based on the metrics of retinal thickness or through the evaluation of the retinal structure using the former [17]. This might change as OCT-A is further evaluated in ongoing studies.

Another important consideration is the advent of widefield imaging combined with FA which has vastly improved our understanding of the role of peripheral vascular changes in driving central macular pathology [18]. Various studies have shown that peripheral ischemia is strongly related to presence and severity of DME as well as recalcitrance to therapy. When widefield imaging is used to calculate the ischemic index, the mean decrease in central macular thickness is highest in the lowest ischemic index group and least in the in the worst ischemic index group [19]. In summary, the information that is obtained from FA on central macular and peripheral retinal changes is critically important for the evaluation of the severity of the disease, aids in staging purposes, and is helpful in the monitoring of outcomes following treatment.

\section{Recommendation}

FA is an important diagnostic tool for assessment of the central and peripheral retina. It is recommended that FA is performed prior to the initiation of therapy to delineate and stage the DME and DR pathology. FA may be repeated as needed in the event of nonresponsiveness to therapy and/or for monitoring patients in the long term. OCT-A may be used to accompany FA imaging for its potential to offer additional insight into capillary loss and attribution to the superficial or deep capillary plexus mainly because of its non-invasive nature.

\section{Features in Optical Coherence Tomography}

\section{Rationale}

Since its first introduction, OCT has become the most frequently used diagnostic tool in ophthalmology and has revolutionized clinical imaging for diagnosis and disease management in most retinal diseases including DME. OCT is a fast, noninvasive technology that produces in vivo images of the retina. The most recent third-generation OCT technology uses a swept-source (SS) light source that allows very fast imaging and provides threedimensional raster images of high microstructural resolution, also referred to as optical histology [20]. The most commonly used second-generation OCT nowadays is SD-OCT allowing three-dimensional raster scans of up to a few hundred B-scans, also creating high-resolution images, but working slower than SS-OCT. It supersedes time-domain (TD)-OCT that allowed imaging of 6 radial cuts only.

All OCT generations are able to generate central retinal thickness (CRT) values, but TD-OCT is less accurate due to its more likely decentration from the fovea and less available B scans. Nevertheless, CRT has been used as a quantitative feature to evaluate disease activity, progression, and treatment response ever since OCT was available in ophthalmology. Although there is a correlation between best-corrected visual acuity (BCVA) letter score and CRT in DME under therapy with anti-VEGF agents, this correlation is weak during the first year of therapy $(r=0.34-0.41)$ and largely lost in the long term [21]. The new OCT generations of SD- and SS-OCT are able to vi-
188

Ophthalmologica 2017;237:185-222 DOI: $10.1159 / 000458539$
Schmidt-Erfurth et al. 


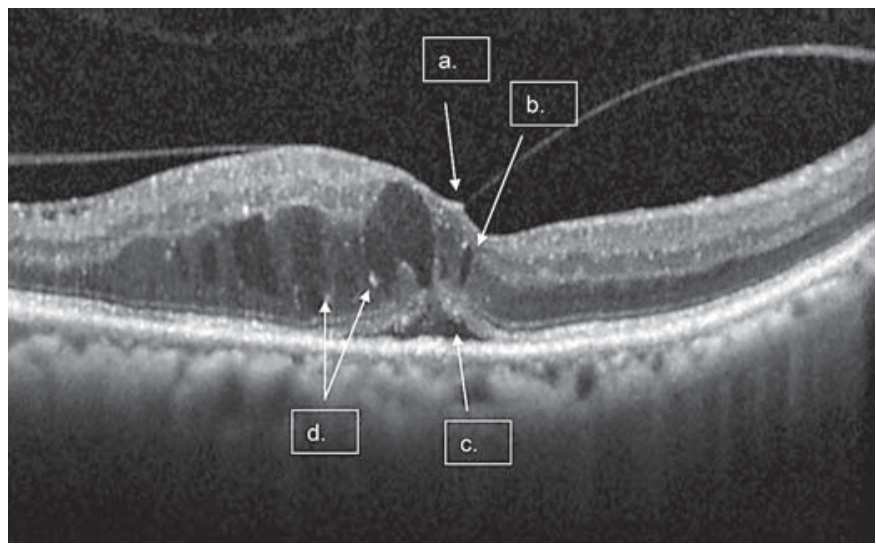

Fig. 3. A single scan with macular fluid and diffuse retina edema. Arrows point to the following features: (a) vitreomacular traction (VMT); (b) intraretinal cystoid fluid (IRC); (c) subretinal fluid (SRF); (d) hyperreflective foci (HRF). Reproduced with permission from Heng et al. [167].

sualize qualitative features in more detail than TD-OCT, among others subretinal fluid (SRF; Fig. 3), intraretinal cystoid fluid (IRC; Fig. 4), disruption or thickness changes of retinal layers (e.g., disorganization of the retinal inner layers [DRIL]; Fig. 5), and the status of the vitreomacular interface (Fig. 3). These devices can also visualize the destructive response of the retina when applying laser [22] (Fig. 6). However, TD-OCT had been used in most large clinical phase III trials evaluating the use of antiVEGF agents in DME (RIDE/RISE [23], RESTORE [2426], DRCR.net protocol I [27]); only few recent large trials have used SD-OCT (VIVID DME/VISTA DME [28], DRCR.net protocol T [29], RETAIN [30]). Advances in OCT like Doppler-OCT and OCT-A (Fig. 7) allow the visualization of vascular structures and can differentiate between perfusion and nonperfusion. Nevertheless, these advances are still lacking the ability of visualizing vascular leakage.

\section{Evidence}

The gold standard in diagnosing DME still remains FA. It can detect different hallmarks of DR like MAs, PDR, ischemic areas and especially DME due to vascular leakage. OCT can be used for screening, classification, monitoring, and treatment evaluation of DME. It has the ability to provide information on CRT as well as distinct morphological features of the edema (Fig. 3-8). Additionally, it can show persistent morphological changes after DME treatment. Morphological signs of de novo, per-

Guidelines for the Management of Diabetic Macular Edema by EURETINA

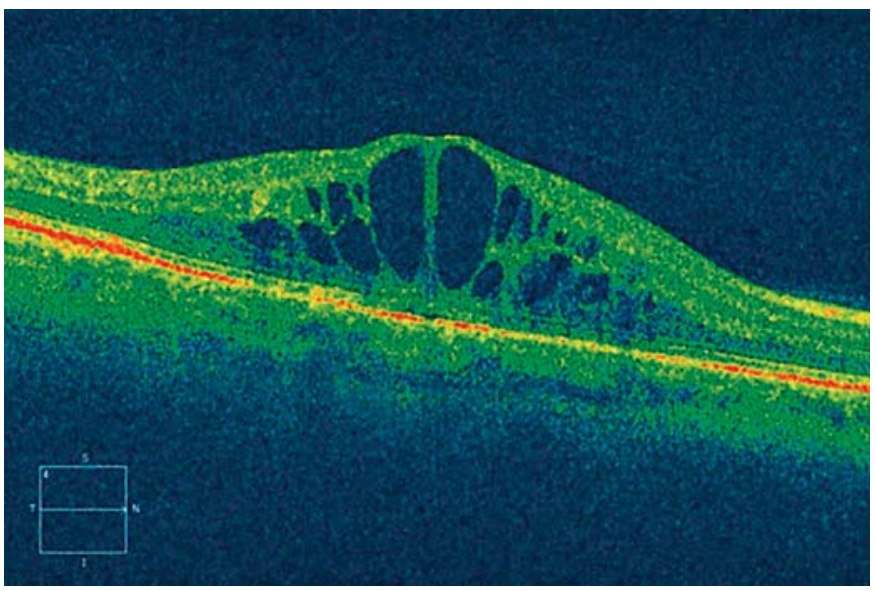

Fig. 4. Cirrus optical coherence tomography image. The pattern of diabetic macular edema indicates cystoid macular edema. Reproduced with permission from Arevalo et al. [168].

sisting, or resolved DME are: SRF (nonreflective space between the neurosensory retina and the retinal pigment epithelium; Fig. 3), IRC (minimally reflective round or oval spaces within the neurosensory retina; Fig. 3 and 4), disorganization of inner retinal layers (DRIL; Fig. 5) [31], other integrity changes of inner and outer photoreceptor segments line (Fig. 8), and external limiting membrane (ELM; Fig. 8) [32], MA, hard exudates/hyperreflective foci (Fig. 3) [33], epiretinal membranes, or changes in choroidal thickness [34]. Many of these features can be seen best (or exclusively) on OCT images and were therefore missed by the early DME guideline studies for laser therapy. The ETDRS was the gold standard for classification and laser therapy planning in DR including, e.g., focal laser for DME for many years [35]. But for anti-VEGF therapy management, evaluation and monitoring of individual treatment responses, OCT is the most used modality today, particularly as OCT captures fluid-pooling as a result of active leaking in DME disease. Therefore, new classification systems for DME were proposed in the past years. One of these is the SAVE protocol that proposes to characterize DME into categories of SRF, area of affected retina by IRC, vitreoretinal interface abnormalities (interface abnormalities).

In large clinical trials to date, CRT was the only OCT criterion used for evaluation. In the RIDE/RISE [23] studies, treatment was performed monthly with intravitreal anti-VEGF, but additional laser treatment was performed on an as needed (PRN) basis based on CRT in OCT. In

Ophthalmologica 2017;237:185-222 189 


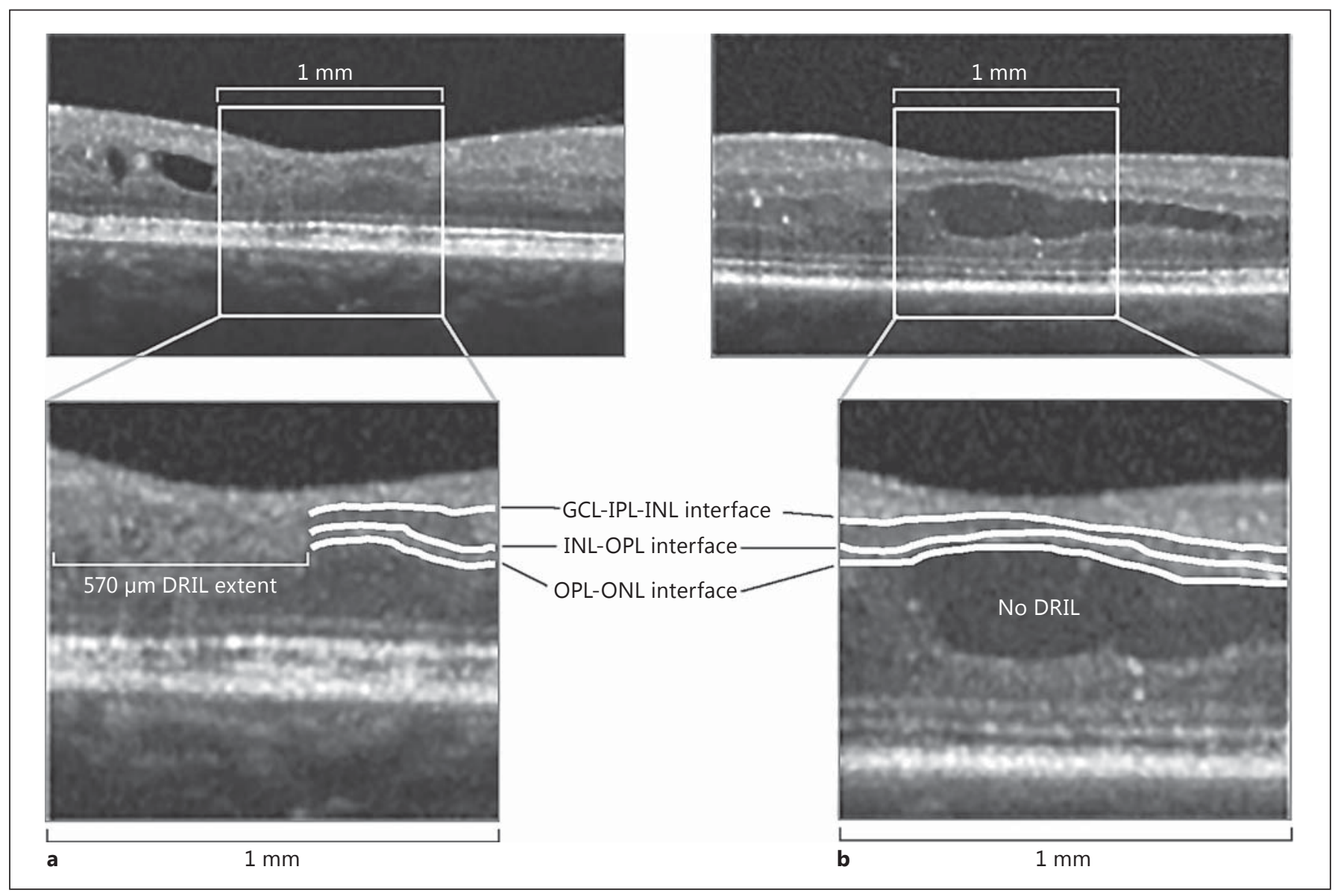

Fig. 5. Representative images of the presence or absence of disorganization of the retinal inner layers (DRIL). a DRIL is present, and retinal layer boundaries can only be partially identified at the right-hand edge of the 1-mm box. $\mathbf{b}$ DRIL is absent, and all retinal layer boundaries can be identified throughout the $1-\mathrm{mm}$ box. The presence or absence of DRIL is independent of other pathology, such as intraretinal cystic changes. Insets are magnifications of the central 1-mm-wide area to show segmentation of the inner retinal layers, with white lines demarcating interfaces between ganglion cell-inner plexiform complex (GCL-IPL), inner nuclear layer (INL), outer plexiform layer (OPL), and outer nuclear layer (ONL). Reproduced from Sun et al. [31] with permission from American Medical Association. the RESTORE [24] study, where intravitreal anti-VEGF monotherapy and laser were compared with a combination therapy of both for DME, retreatment was based on BCVA letter score and the investigator's decision which could include OCT changes and clinical examination. In the DRCR.net protocol I [27] intravitreal anti-VEGF injections were compared with laser treatment and with intravitreal corticosteroid injections. In this study, retreatment was also based on the investigator's discretion without predefined OCT retreatment criteria. In the VIVID DME/VISTA DME [28] studies with SD-OCT, a fixed treatment regimen was used for intravitreal anti-VEGF therapy, and laser retreatment was allowed as indicated by ETDRS guidelines. CRT was used as the secondary efficacy endpoint. In the DRCR.net protocol T [29], CRT from OCT was one parameter for retreatment decisions. In conclusion, all these trials show that BCVA played the most important role with regard to retreatment; therefore, CRT as an OCT parameter has played little role in the study design and analyses of most large clinical phase III trials on DME. However, ophthalmologists to date agree that treatment regimens must be individualized because OCT biomarkers are key to identify the best PRN treatment scheme for each individual patient. A PRN regimen is the most frequently used treatment regimen for DME in anti-VEGF therapy in real life today, but the PRN
Schmidt-Erfurth et al. 
Fig. 6. Immediate morphologic changes after grid photocoagulation. Morphologic findings before (first line) and 1 day after (middle line) laser treatment are shown in infrared imaging (left) and OCT (right). The green line in the infrared image indicates the position of the scan performed exactly at the same position in both examinations. The red boxes indicate the areas ( $a$, b) magnified in the lower line. Laser lesion site (red arrows) at the level of the pigment epithelium. The blue lines in the magnifications indicate the characteristic angulated alteration pattern in each single laser lesion. ELM, external limiting membrane; INL, inner nuclear layer; ONL, outer nuclear layer; OPL, outer plexiform layer; PRL, photoreceptor layer. Reprinted from Bolz et al. [22] with permission from Elsevier.

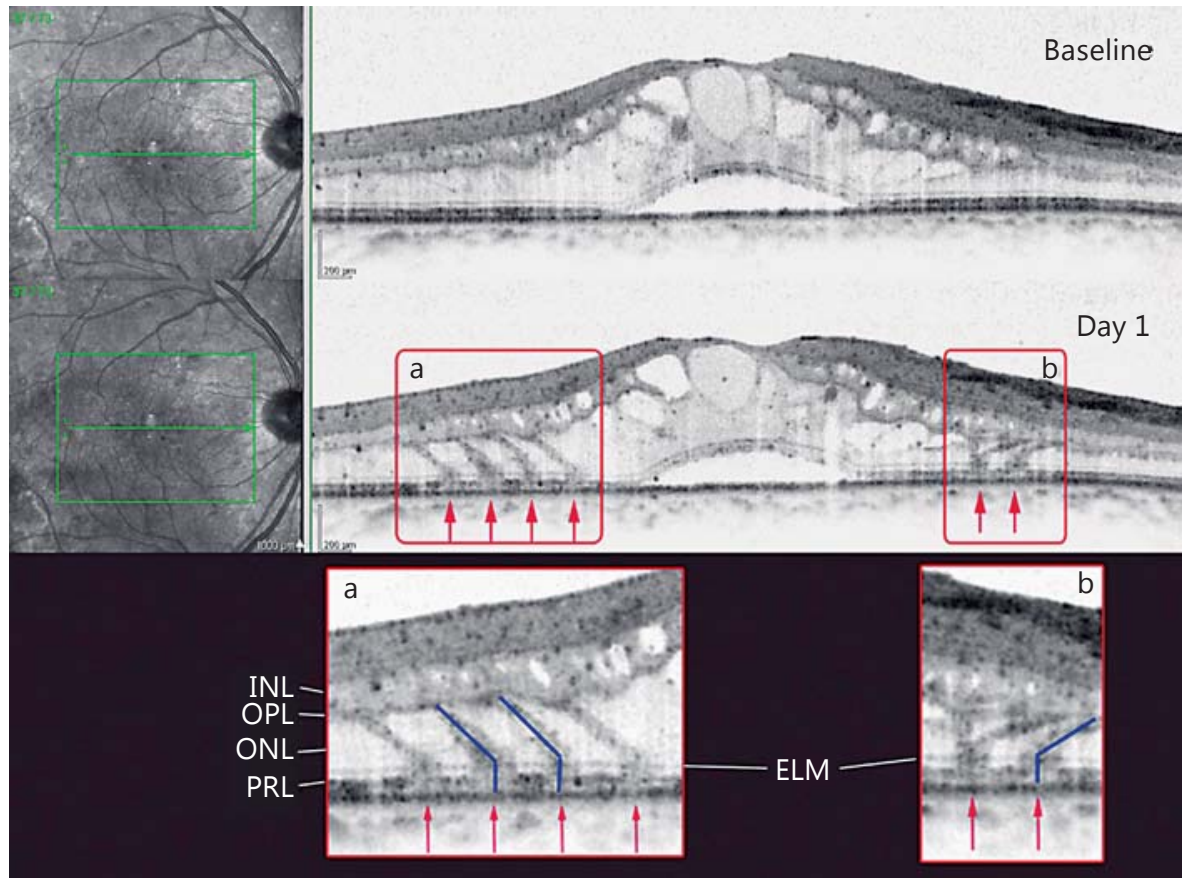

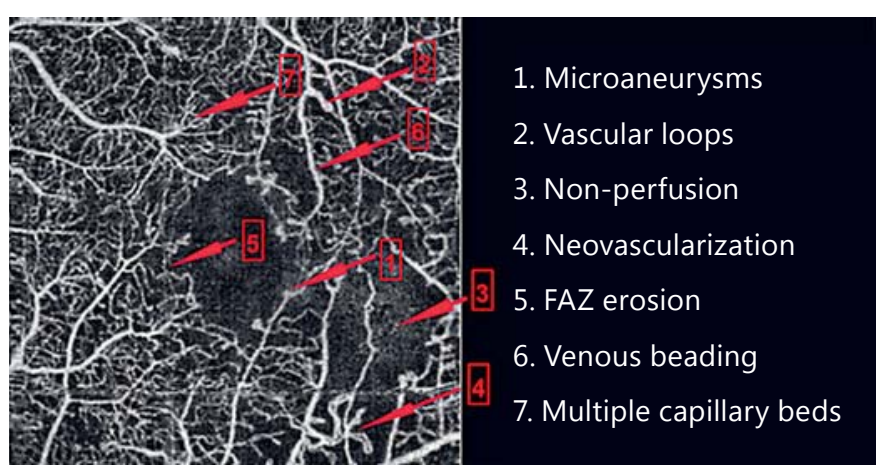

Fig. 7. Features of diabetic retinopathy visible with optical coherence tomography angiography. Reprinted from Lee et al. [169] with permission from Springer.

criteria are not standardized. The individual patient's morphology has a close relationship with the treatment response and needs to serve as the basis for these criteria. Focal edema classified with the earlier described system, for example, may benefit most from anti-VEGF therapy with regard to BCVA letter score gain [36]. Some individual OCT biomarkers have already been identified from post hoc analyses of the multicenter studies and some smaller monocentric studies.

Guidelines for the Management of Diabetic Macular Edema by EURETINA
In the RESTORE study, patients have been treated after an initial loading phase of 3 consecutive monthly injections on a PRN basis. Here, it could be shown in a post hoc analysis that patients with SRF at baseline had higher BCVA gains at the end of the first study year than patients without SRF at baseline, although no difference in their baseline BCVA letter score was detected [21]. This protective role of SRF was reconfirmed by an OCT post hoc study analysis of the RIDE/RISE trials [37]. When looking at IRC, in RESTORE about $80 \%$ of patients had IRC at baseline. Patients with less IRC at baseline had a better baseline BCVA letter score and remained at a better score throughout the entire study duration [21]. This finding is supported by an analysis of another study of preserved tissue in $129 \mathrm{DME}$ patients. Here, baseline vision correlated with the volume of preserved neurosensory tissue is less IRC between inner and outer retina [38]. Another finding from RESTORE indicated that throughout the entire study duration, patients with vitreomacular adhesion showed higher BCVA scores at baseline and maintained these better scores under any therapy, whereas patients with a posterior vitreous detachment started with lower BCVA letter scores and improved less than patients with vitreomacular adhesion [21].

In an academic study with 120 patients, the importance of DRIL was shown. DRIL that constituted more than $50 \%$ of the central millimeter on OCT was associ-

Ophthalmologica 2017;237:185-222 191 


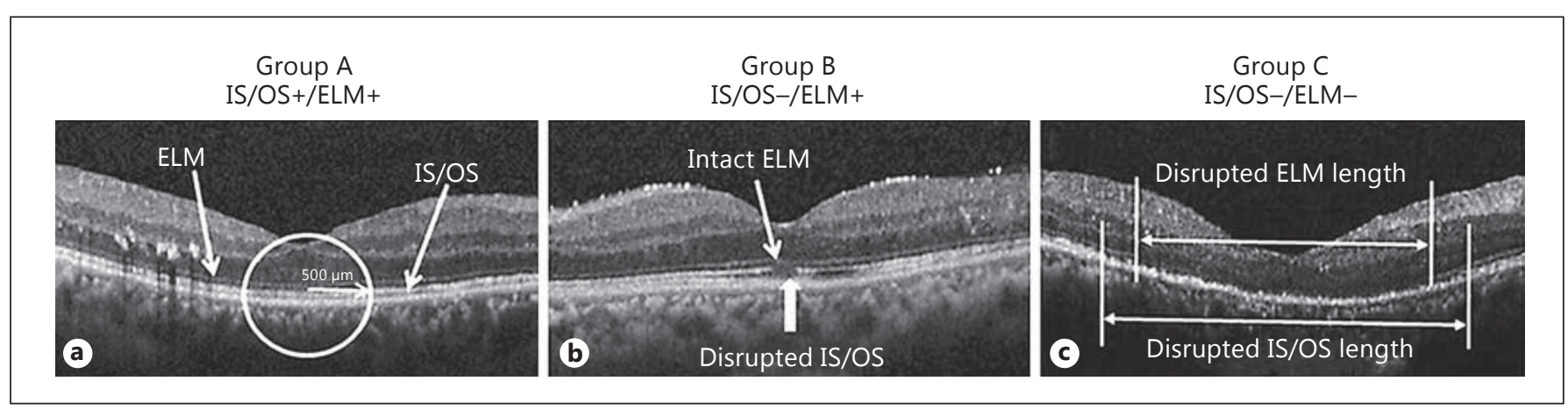

Fig. 8. The pattern of the photoreceptor inner and outer segment junction (IS/OS) and external limiting membrane (ELM) within $500 \mu \mathrm{m}$ from the center of the fovea at the final visit obtained by SD OCT after resolution of diabetic macular edema. a Completely visible IS/OS and ELM (IS/OS+/ELM+). b Disrupted IS/OS and intact ELM (IS/OS-/ELM+). c Disruption or loss of IS/OS and ELM (IS/OS-/ELM-). Reprinted from Shin et al. [32] with permission from Springer. ated with a worse BCVA score in current and resolved edema; DRIL was able to predict a worse change in BCVA score until the end of the first year of treatment if it occurred in the first 4 months of treatment [39] (Fig. 5).

When monitoring anti-VEGF therapy responses, the thickness of the ganglion cell layer (GCL), retinal nerve fiber layer (RNFL), and choroidal layer may decrease; a correlation with vision loss could only be shown for the GCL, whereas a decrease in choroidal thickness during therapy does not correlate with less vision gain [40-42]. On the other hand, a thick subfoveal choroid at baseline may predict good vision outcomes [43]. The peripapillary RNFL is thickened in all patients with DME before and after anti-VEGF therapy compared to non-DME patients with signs of DR, although it decreases after anti-VEGF [42]. The appearance of hyperreflective foci (Fig. 3) in OCT may precede formation of hard exudates that can be seen in fundus biomicroscopy. During anti-VEGF therapy, hard exudates increase in number and size [44, 45].

When looking at OCT changes after laser photocoagulation therapy, one day after laser application an oblique disruption of the outer retinal layers (retinal pigment epithelium, photoreceptor layer, and outer nuclear layer) can be observed [22] (Fig. 6). This disruption is repaired in about $50 \%$ of the cases within 3 months after photocoagulation therapy, but clearly highlights the destructive nature of photocoagulation [46].

These morphological analyses show that excellent results can be achieved for a PRN regimen in DME, with a decreasing number of injections during the disease course. In the RESTORE Extension Study, vision im- provement could be maintained in year 2 (3) with less than 4 (3) mean injections compared to 7-8 injections in the first year of the study even with the use of CRT changes as the only diagnostic criterion. Other than in age-related macular degeneration, where a continuous injection therapy is necessary, the disease activity seems to plateau in DME, which is explained by a diseasemodifying mechanism of VEGF inhibition. This is also confirmed by the DRCR.net protocol I, where BCVA score improvement could be maintained for 5 years with fewer than 5 anti-VEGF injections in years 3-5 (about one-quarter of patients having no more injections in year 2, one-third in year 3, and half no more injections in years 4 and 5 of the study) $[47,48]$. Patients had only been treated with anti-VEGF for 6 months and discontinued if no more change could be seen (improvement or worsening) - even if retinal fluid persisted [27]. The examples of multimodal imaging in patients with different therapy responses are presented in Figures 9 and 10. Compared to age-related macular degeneration where a "no tolerance" retreatment is usually used (CATT, HARBOR) and recommended [49], this means that a restrained treatment frequency based on BCVA and OCT monitoring can be chosen in DME. Further analyses must prove if a certain morphology pattern can be identified for patients who lose vision after discontinuing treatment in persisting DME after 6 months. Here, especially high resolution SDand SS-OCT with its tight B-scan spacing will be of great value. The ability of imaging the vascular network with Doppler-OCT and OCT-A (Fig. 7) will further increase the insight in DME as a vascular disease and may
192
Ophthalmologica 2017;237:185-222 DOI: $10.1159 / 000458539$
Schmidt-Erfurth et al. 

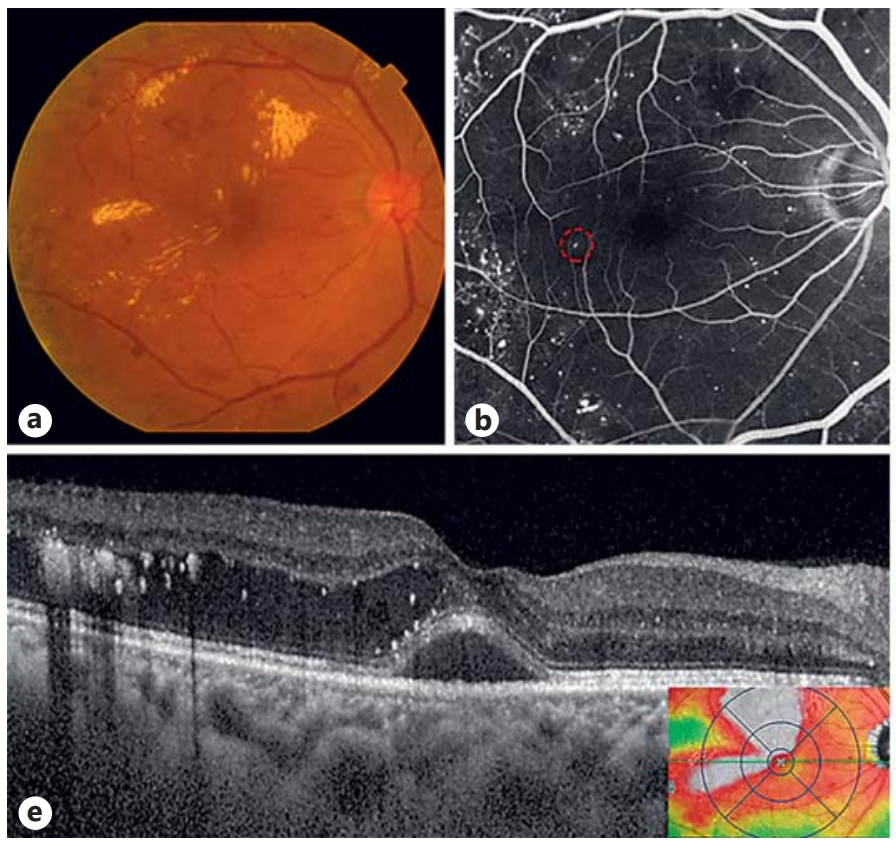

Fig. 9. A diabetic macular edema (DME) eye showing a good response to anti-vascular endothelial growth factor treatment. Baseline fundus photography (a) and fluorescein angiography (FA) (b) of the eye. Optical coherence tomography angiography showing multiple microaneurysms in the deep capillary plexus (d), but none in the superficial capillary plexus (c). Yellow circles indicate

deliver new hypotheses on imaging biomarkers. Individualized retreatment criteria based on OCT biomarkers may be established in the future.

\section{Recommendation}

In order to diagnose DME, OCT should be accompanied by baseline FA and continued fundus biomicroscopy. When diagnosed and treated, a recurrence of edema can usually be monitored solely by OCT in combination with visual acuity measurements. Robust OCT biomarkers need to be reassured in large-scale clinical studies, but evidence indicates that good baseline predictors for a good treatment response with regard to high vision gains and/or good final visual acuity are SRF and/ or small IRC and/or vitreomacular adhesion at baseline $[21,50]$. The disorganization or disruption of the inner retinal layers [31], disruption of the inner and outer photoreceptor segments and/or ELM [32], and a thin subfoveal choroid at baseline [43] may predict bad visual acuity after therapy.

Findings in OCT include qualitative and quantitative measures. CRT is an established quantitative measure-

Guidelines for the Management of Diabetic Macular Edema by EURETINA


microaneurysms that were not depicted on FA. Red circles indicate a microaneurysm that is also seen on the FA images. Compared with the baseline spectral-domain optical coherence tomography (SD OCT) (e), the follow-up SD OCT (f) showed improved macular edema after 3 bevacizumab injections. Reprinted from Lee et al. [170].

ment. As a CRT increase consists of SRF and/or IRC and/or diffuse thickening, distinguishing between the different qualities may be useful. PRN treatment based solely on CRT changes is a practical approach for the management of DME; other promising more robust individual OCT features have to be confirmed before they can feed into retreatment decisions. The next development will likely allow the quantification of these qualitative biomarkers. Segmentation of retinal layers, IRC and SRF and volumetric computation for these features may have an impact on individualized PRN regimens $[51,52]$.

Once criteria are validated, most recent OCT technology with high resolution and dense scanning will help three-dimensional image analysis to be automated and to detect disease activity as smallest morphological changes early on. Therefore, the optimal recommendation is to monitor disease activity on a monthly basis with OCT even if no treatment is needed or intended in order to identify morphological changes as early as possible. These recommendations are based on evidence levels I and II.

Ophthalmologica 2017;237:185-222 

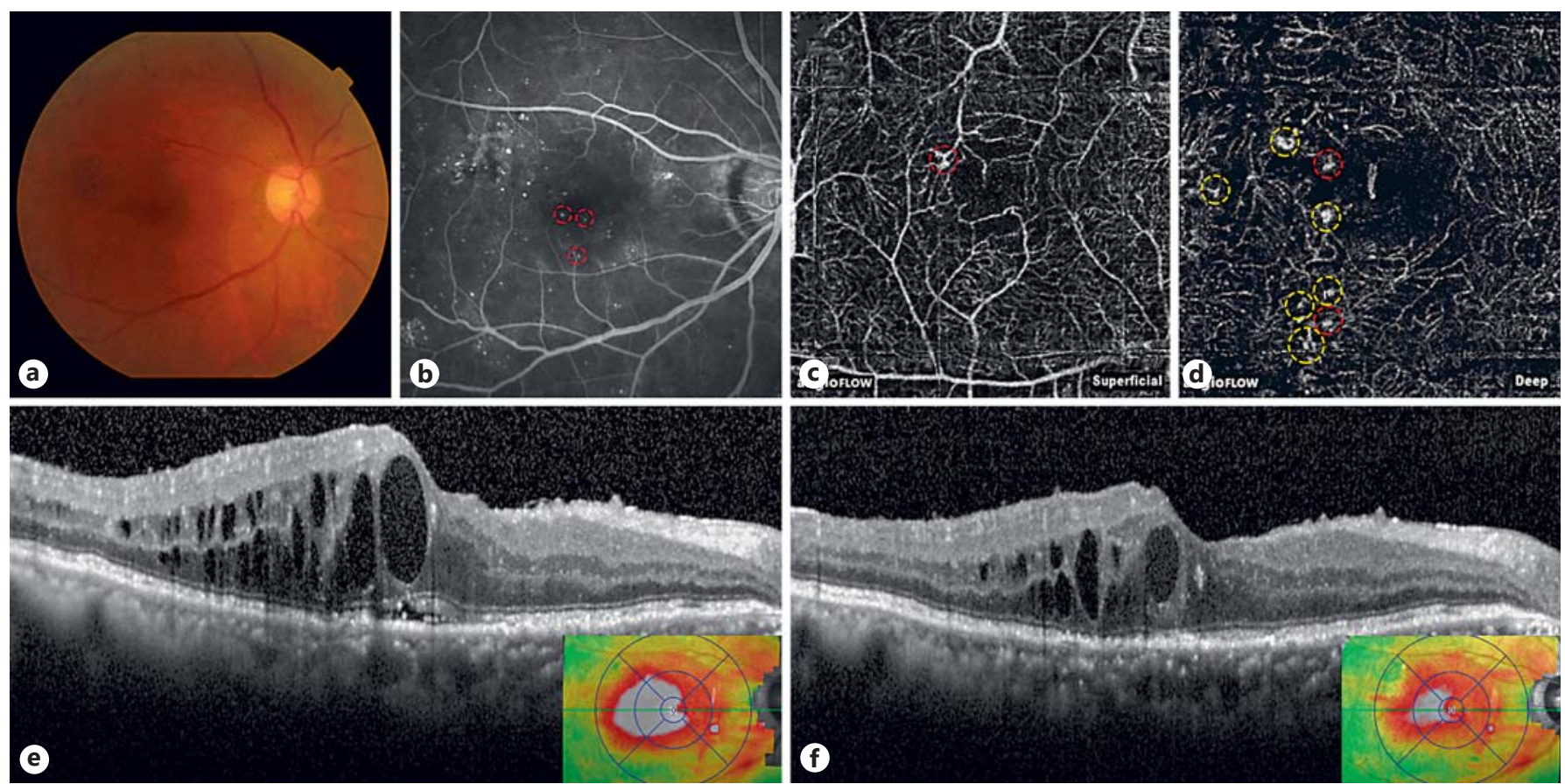

Fig. 10. A diabetic macular edema (DME) eye showing a good response to anti-vascular endothelial growth factor treatment. Baseline fundus photography (a) and fluorescein angiography (FA) (b) of the eye. Optical coherence tomography angiography showing multiple microaneurysms in the deep capillary plexus (d), but none in the superficial capillary plexus (c). Yellow circles indicate

\section{Therapeutic Strategies}

\section{Laser Therapy}

Rationale

Laser photocoagulation has represented the standard of care for the treatment of DME prior to the advent of the intravitreal injection approach. The efficacy of focal laser treatment has been related to the occlusion of leaking vessels, especially MA, but the exact mechanism by which focal photocoagulation reduces DME is unknown. The mechanism of action of laser is mainly based on the destruction of ischemic retina, leading to improved oxygenation of neighboring retinal areas, reduced production of proangiogenetic factors, and also release of cytokines from the retinal pigment epithelium and Muller cells. Different types of laser can be used, including Argon green (514 nm), dye yellow (577 nm), Krypton red (647 $\mathrm{nm})$, and diode (810 $\mathrm{nm}$ ) laser.

microaneurysms that were not depicted on FA. Red circles indicate a microaneurysm that is also seen on the FA images. Compared with the baseline spectral-domain optical coherence tomography (SD OCT) (e), the follow-up SD OCT (f) showed improved macular edema after 3 bevacizumab injections. Reprinted from Lee et al. [170].

\section{Evidence}

The ETDRS specifically explored the benefits of laser treatment in DME. Laser photocoagulation was prescribed for all lesions located within 2 disc diameters of the macular center. The results at 3 years demonstrated that patients who were treated with focal photocoagulation for clinically significant macular edema achieved a $50 \%$ reduction in the risk of moderate visual loss (losing $>15$ letters of BCVA) compared to controls (12-24\%) [11, 53]. Unfortunately, only $3 \%$ of patients showed a visual acuity improvement of 3 or more lines. Focal laser energy application should be addressed to leaking MA in combination with grid laser treatment of areas of diffuse macular leakage and nonperfusion in thickened retinas. Complications associated with conventional lasers include color vision, night vision, and contrast sensitivity impairment, along with enlargement of laser scars, secondary choroidal neovascularization, subretinal fibrosis, and visual-field sensitivity deterioration.

Another randomized clinical trial, Protocol B of the DRCR.net, confirmed the positive effects of focal/grid 
Fig. 11. DRCR.net Protocol I study. Mean change in visual acuity at follow-up visits. Open triangle $($ prompt $)=$ ranibizumab + prompt laser treatment; closed square (deferred $)=$ ranibizumab + deferred laser treatment. Visual acuity change truncated to \pm 30 letters. Results were similar without truncation (data not shown). Difference in mean change in visual acuity at 5 years from longitudinal model: $p$ value adjusted for baseline visual acuity $=0.09$; $p$ value adjusted for baseline visual acuity and other potential confounders $=0.15$. Reprinted from Elman et al. [48] with permission from Elsevier.

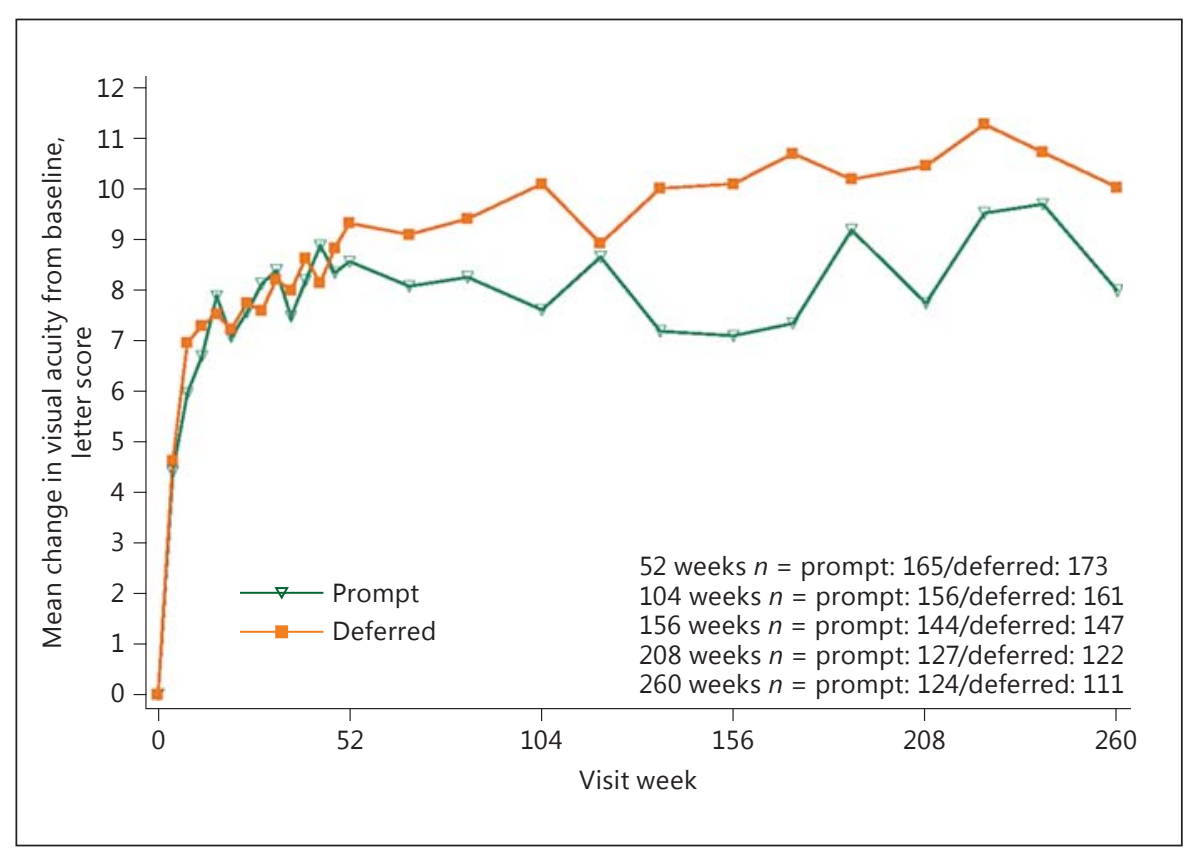

photocoagulation, demonstrating that in phakic patients laser was more effective than intravitreal injection of triamcinolone acetonide in DME patients at both 2 and 3 years of follow-up [54, 55].

A more recent laser application is subthreshold grid laser treatment, which has been proposed in an attempt to minimize the destructive aspects of conventional grid laser photocoagulation, obtaining encouraging results [56]. The fundamental concept of subthreshold grid laser treatment is to reduce the laser damage to neurosensory layers by reducing the duration of light exposure and by using a subvisible clinical endpoint. In particular, the micropulse diode laser allows the release of micropulses with low energy per pulse in order to confine the energy to the cells of the retinal pigment epithelium (RPE), avoiding lateral thermal spreading. A few randomized clinical trials have demonstrated that subthreshold grid laser treatment is as effective as conventional focal/grid laser photocoagulation, even though slower in terms of resolution of DME, in achieving the same functional and anatomical effects $[57,58]$.

During the era of anti-VEGF, the DRCR.net Protocol I study was performed to evaluate if prompt or deferred laser in addition to ranibizumab therapy would be of benefit with regard to BCVA gain after 5 years of treatment. The results showed that among eyes treated with ranibizumab, results were similar after 12 months whether laser was given starting with the first injection or by 24 weeks

Guidelines for the Management of Diabetic Macular Edema by EURETINA deferred [27]. But deferring laser led to a superior area under the curve (AOC) regarding BCVA (Fig. 11), especially in eyes with BCVA at baseline lower than 69 letters (Fig. 12) and the number of patients achieving a $>15$ letter BCVA gain was higher in patients with deferred laser therapy [48]. Laser therapy has therefore been taken from the position of first-line recommendation and is rather considered to be harmful by many retina experts. Refer to the different sections of injection therapy to find evidence on the efficacy of laser versus anti-VEGF or steroid injection therapy.

In the presence of DME and high-risk non-PDR or PDR, DRCR.net Protocol S [59] data clearly revealed superiority of anti-VEGF (ranibizumab) therapy as the sole intervention which improves BCVA and additionally induces regression of PDR.

\section{Recommendation}

Laser treatment has been shown to be an effective treatment option in the management of DME in the early pioneering studies compared to sham treatment, but is currently in the era of anti-VEGF injection clearly not the standard of care anymore. Relative indications include laser application especially to the vasogenic subform of DME, which is clinically characterized by the presence of focally grouped MA and leaking capillaries. A further indication is represented by eyes affected by DME with CRT less than $300 \mu \mathrm{m}$ or eyes with persisting vitreomacu-

Ophthalmologica 2017;237:185-222 195 
Fig. 12. DRCR.net Protocol I study. Mean change in visual acuity (VA) at follow-up visits stratified by baseline VA subgroup. Open triangle $($ prompt $)=$ ranibizumab + prompt laser treatment; closed square (deferred $)=$ ranibizumab + deferred laser treatment. VA change truncated to \pm 30 letters. $p$ value for interaction of treatment group with baseline VA over 5 years from the longitudinal model $=0.004$. Reprinted from Elman et al. [48] with permission from Elsevier.



lar adhesion, because comparable results can be achieved by means of laser photocoagulation or anti-VEGF injections [24]. Subthreshold grid laser treatment can be helpful in eyes with higher visual acuity affected by early diffuse DME, in order to avoid the collateral thermal diffusion and the consequent chorioretinal damage as a less expensive option. In general, there is no support for the evidence that laser would add more benefit than pharmacotherapy, but rather reduce the gain. Economic consid- erations based on the costs of anti-VEGF substances or the lower number of interventions and monitoring visits with laser do not outweigh the disadvantages of laser in DME.

\section{Anti-VEGF Agents}

Bevacizumab

Rationale. Bevacizumab (Avastin) is a full-length, humanized, monoclonal antibody that binds to and inhibits 
Fig. 13. DRCR.net Protocol T study. Mean change in visual acuity over time: shown are the changes in visual acuity overall (a) and according to baseline visual acuity (b). b Solid lines indicate baseline visual acuity of $20 / 50$ or worse, and dashed lines indicate baseline visual acuity of $20 / 32$ to $20 / 40$. Outlying values were truncated to $3 \mathrm{SD}$ from the mean. The number of eyes assessed at each 4-week interval ranged from 195 to 224 in the aflibercept group, 188218 in the bevacizumab group, and 188218 in the ranibizumab group (see Fig. S2 in the Supplementary Appendix of the cited publication for the exact number assessed at each 4-week interval). T bars indicate 95\% confidence intervals. Reprinted from Wells et al. [29] with permission from Massachusetts Medical Society.
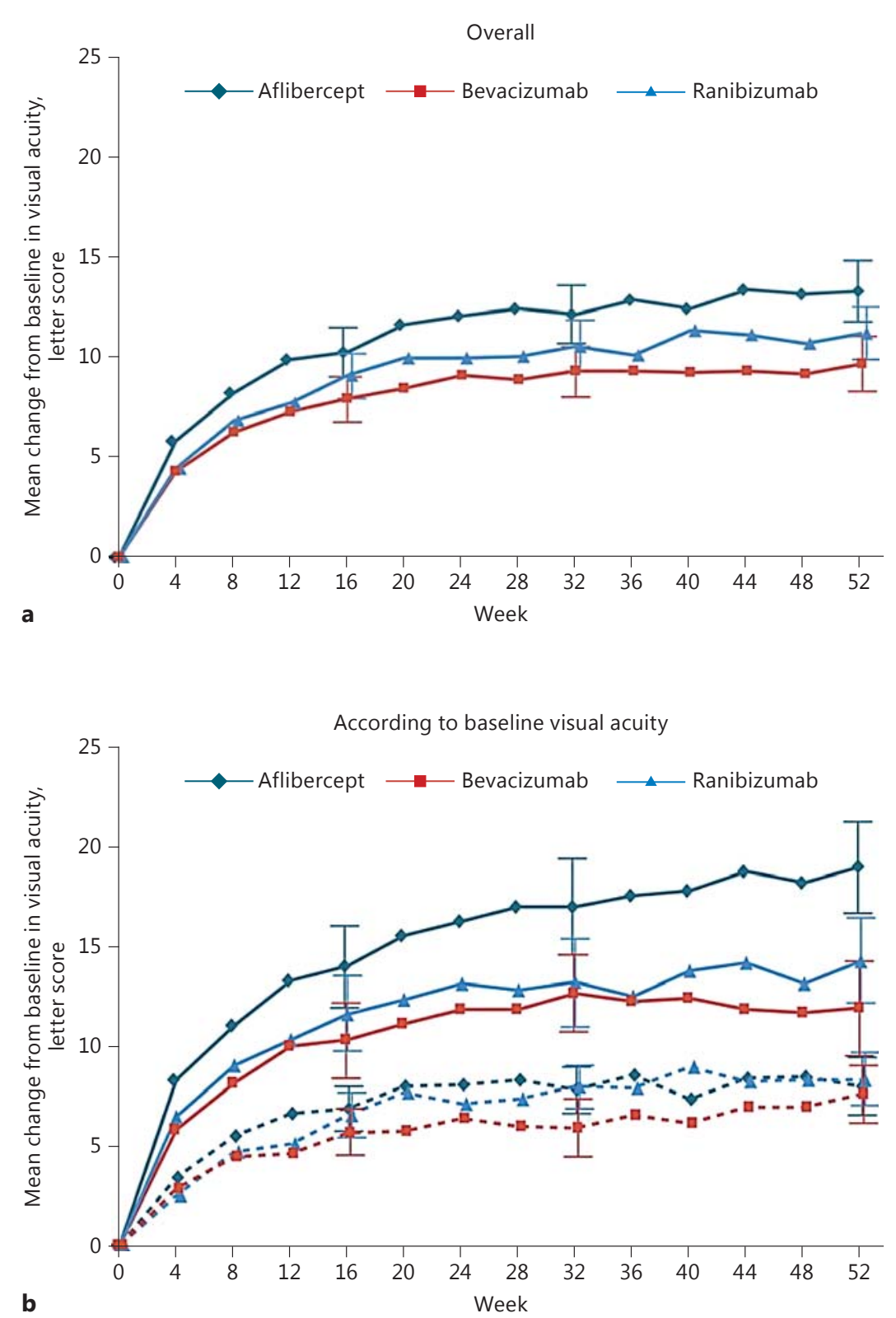

all VEGF isoforms. The molecule was developed to reduce tumor growth by inhibiting pathological tumor vessel formation in metastatic colon cancer [60]. The medication has received approval by the Food and Drug Administration (FDA) and the European Medicines Agency (EMA) for systemic treatment of several cancer forms. In oncology, treatment is administered as systemic infusions at doses of $5-15 \mathrm{mg} / \mathrm{kg}$ every 2 or 3 weeks with known increased risk of thromboembolic events, hypertension, hemorrhages, and gastrointestinal perforation. Bevacizumab was designed for a prolonged retention in serum. The Fc region of the bevacizumab antibody binds to $\mathrm{FcRn}$ receptors that are highly expressed in vascular endothelial cells. The molecule is thereby protected from proteolytic catabolism and recycled systemically [61]. Based on a pharmacokinetic analysis of 491 patients who received 1-20 mg/kg of bevacizumab weekly, every 2 or every 3 weeks, the estimated half-life of bevacizumab in serum
Guidelines for the Management of Diabetic Macular Edema by EURETINA
Ophthalmologica 2017;237:185-222 DOI: $10.1159 / 000458539$ 
was approximately 20 days, ranging from 11 to 50 days. When used off-label for ocular disease, the standard dose of intraocular bevacizumab is $1.25 \mathrm{mg}$ in $0.05 \mathrm{~mL}$. Intravitreal half-life of bevacizumab in human vitreous samples after one single injection ranged from 3 to 6.7 days [62]. Systemic retention after intravitreal treatment with bevacizumab was measured in patients with DME, with significantly reduced systemic plasma levels of VEGF up to 4 weeks after a single bevacizumab injection $[63,64]$.

Intraocular bevacizumab is widely used as an off-label treatment for neovascular age-related macular degeneration (nAMD) and DME. Bevacizumab vials can be divided into multiple dosages and the cost is manifold lower compared to ranibizumab and aflibercept. Since bevacizumab is not approved for the treatment of ocular disease, there has been a pressing need for robust comparative data of efficacy and safety.

Evidence. In March 2015, the DRCR.net published the first-year results of the Protocol T study. Protocol T is a randomized clinical trial, sponsored by the National Institutes of Health (NIH) [29]. The purpose was to compare efficacy and safety of intravitreal aflibercept, bevacizumab, and ranibizumab for the treatment of DME involving the center of the macula with vision loss. The study included 660 patients at a mean age of $61 \pm 10$ years, of which $90 \%$ had diabetes type 2 . The mean visual acuity at baseline was $64.8 \pm 11.3$ letters (Snellen equivalent, approximately 20/50), and the mean CRT was $412 \pm 130 \mu \mathrm{m}$. The completion of the 1-year visit was $96 \%$.

At 1 year, the mean BCVA letter score improved by 13.3 letters when treated with aflibercept, 9.7 letters with bevacizumab, and 11.2 letters with ranibizumab. The overall better results for aflibercept in the general population were, however, not considered relevant due to interaction between baseline BCVA letter score and treatment results. When the initial BCVA score was 69 letters or more (Snellen equivalent, 20/40 or better), the mean BCVA letter score improved by $8.0 \pm 7.6$ for aflibercept, $7.5 \pm 7.4$ for bevacizumab and $8.3 \pm 6.8$ for ranibizumab, with no statistically significant difference between the treatment groups. For study eyes with an initial BCVA score of less than 69 letters (Snellen equivalent 20/50 or worse), the mean improvement was $18.9 \pm 11.5$ for aflibercept, $11.8 \pm 12.0$ for bevacizumab, and $14.2 \pm 10.6$ for ranibizumab. Between the treatment groups with this lower baseline BCVA, there was a clear significant difference in favor of aflibercept, compared to bevacizumab and ranibizumab ( $p=0.0001$ for aflibercept vs. bevacizumab, $p=$ 0.0003 for aflibercept vs. ranibizumab, $p=0.21$ for ranibizumab vs. bevacizumab; Fig. 13). The median number of intravitreal injections needed to achieve the BCVA gain was 9 (interquartile range $8-11$ ) in the aflibercept group, $10(8-12)$ in the bevacizumab group and $10(8-11)$ in the ranibizumab group. The greatest mean reduction in CRT was $169 \pm 139 \mu \mathrm{m}$ for eyes treated with aflibercept, while the CRT was reduced by $101 \pm 121 \mu \mathrm{m}$ for bevacizumab and $147 \pm 134 \mu \mathrm{m}$ for ranibizumab (intravitreal injections needed to achieve the BCVA gain was 9 [interquartile range 8-11] in the aflibercept group, 10 [8-12] in the bevacizumab group, and 10 [8-11] in the ranibizumab group) supporting a superior efficacy of aflibercept.

For the 2-year results of Protocol T [65], the findings slightly changed. BCVA gain at 2 years was 12.8 letters in the aflibercept, 10.0 letters in the bevacizumab, and 12.3 letters in the ranibizumab group (pairwise comparisons: $p=0.02$ for aflibercept vs. bevacizumab, $p=0.47$ for aflibercept vs. ranibizumab, and $p=0.11$ for ranibizumab vs. bevacizumab), suggesting that ranibizumab caught up its overall difference in BCVA gain compared to aflibercept, but bevacizumab did not. The median number of intravitreal injections needed in the second year to maintain the BCVA gain was $5(2-7)$ in the aflibercept group, $6(2-9)$ in the bevacizumab group, and $6(2-9)$ in the ranibizumab group. The greatest mean reduction in CRT was $171 \pm 141 \mu \mathrm{m}$ for eyes treated with aflibercept, while the CRT was reduced by $126 \pm 143 \mu \mathrm{m}$ for bevacizumab and $149 \pm 141 \mu \mathrm{m}$ for ranibizumab, with a statistically significant difference between bevacizumab and the other two drugs. The percentages of eyes undergoing at least one session of laser photocoagulation during the two years were 41,64 , and $52 \%$ in the aflibercept, bevacizumab, and ranibizumab groups.

Dividing patients again into subgroups by baseline visual acuity, the subgroup with a better BCVA letter score (>69 letters) showed no statistical significance between the drugs, like in year one. The subgroup with less than 69 letters at baseline showed a mean BCVA letter score improvement to two years of $+18.1 \pm 13.8$ letters (aflibercept),$+13.3 \pm 13.4$ letters (bevacizumab) and $+16.1 \pm 12.1$ letters (ranibizumab) (aflibercept vs. bevacizumab, $p=$ 0.02 ; aflibercept vs. ranibizumab, $p=0.18$; ranibizumab vs. bevacizumab, $p=0.18$ ), demonstrating that both aflibercept and ranibizumab remain the better choice for patients with poor baseline visual acuity (Fig. 14).

Safety concerns regarding intravitreal bevacizumab have been an issue for many ophthalmologists over the last decade. Systemic exposure by the serum is known to be higher for bevacizumab and aflibercept compared to ranibizumab. The clinical significance of this is still unclear. The trial comparing aflibercept, bevacizumab, and
198

Ophthalmologica 2017;237:185-222 DOI: $10.1159 / 000458539$
Schmidt-Erfurth et al. 


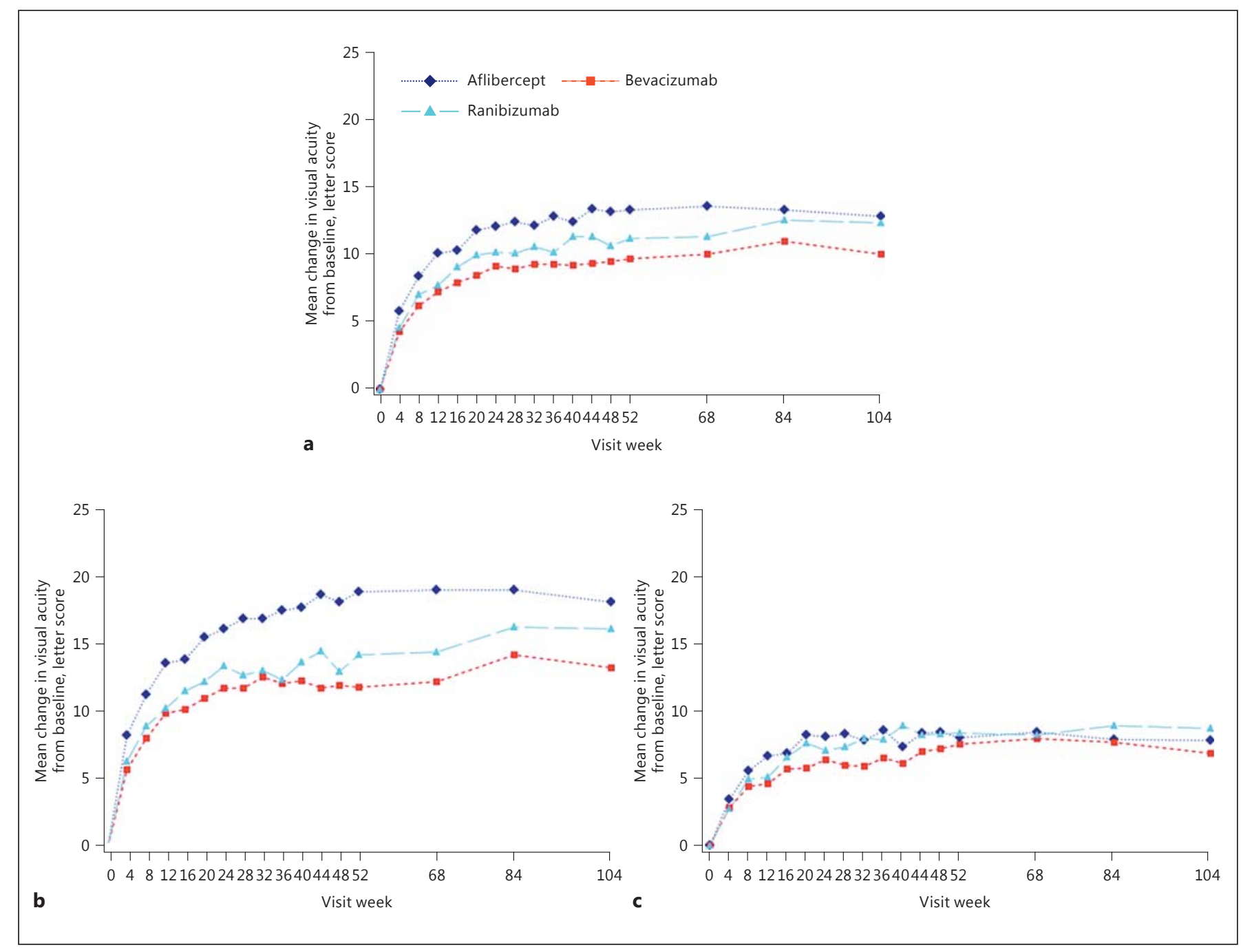

Fig. 14. DRCR.net Protocol T study. Graphs show the mean change in visual acuity over time stratified by baseline visual acuity (approximate Snellen equivalent): overall (a), 20/50 or worse (b), and $20 / 32$ to $20 / 40$ (c). Change in visual acuity was truncated to 3 standard deviations from the mean. The number of eyes at each time point ranged from 195 to 224 in the aflibercept group, 185-218 in the bevacizumab group, and 188-218 in the ranibizumab group (see Fig. S1 in the Supplementary Appendix and Fig. S2 in the 1 Year Supplementary Appendix 2 of the cited publication for the number at each time point; available at www.aaojournal.org). Reprinted from Wells et al. [65] with permission from Elsevier. ranibizumab for the treatment of DME demonstrated more cardiovascular events in the ranibizumab group compared to the aflibercept and bevacizumab groups. This finding is not consistent with data from previous trials with ranibizumab for DME [26, 28, 66-69]. Since the study was not powered to detect differences in adverse events between the medications, the observations might have been due to chance. Multicenter trials (including a Cochrane database systematic review) comparing bevacizumab and ranibizumab for the treatment of nAMD did

Guidelines for the Management of Diabetic Macular Edema by EURETINA not reveal any significant differences in safety between the two medications [49, 70-73]. Diabetic patients, however, may differ from patients with nAMD, and safety data from patients with nAMD might therefore not be directly applicable to diabetic patients. Efficacy and safety of bevacizumab for the treatment of DME remains to be further explored.

Recommendation. The comparative trial from the DRCR.net has provided data about efficacy and safety for bevacizumab, aflibercept, and ranibizumab. According to 


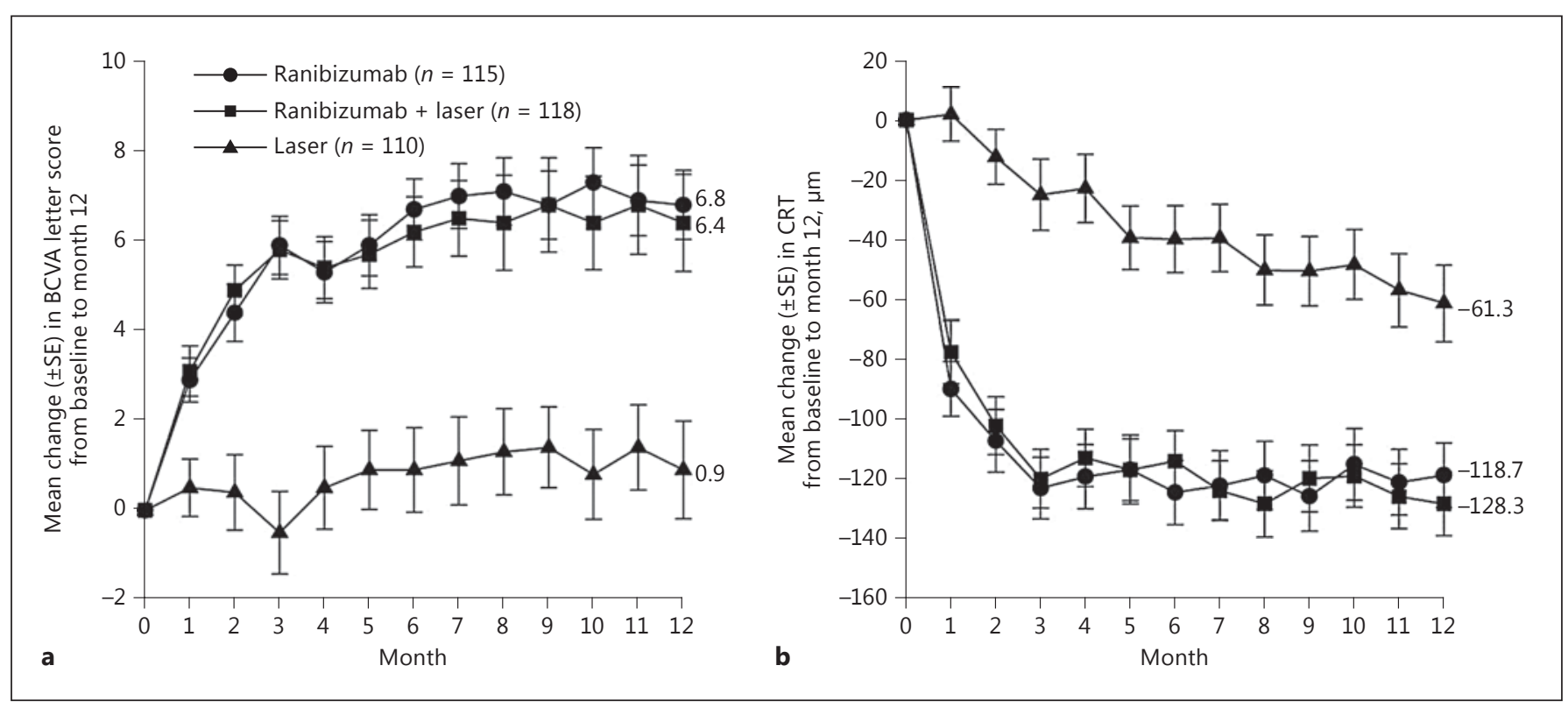

Fig. 15. RESTORE study. a Mean change in best-corrected visual acuity (BCVA) letter score from baseline to month 12. b Mean change in central retinal thickness (CRT) from baseline to month 12. SE, standard error. Reprinted from Mitchell et al. [24] with permission from Elsevier.

these results, the choice of treatment for DME depends on the baseline BCVA letter score. While aflibercept and ranibizumab are the drugs of choice for BCVA letter score of less than 69, all three medications are equivalent in improving vision in eyes with a baseline BCVA letter score of 69 or more. The numbers of serious adverse events were altogether small, but the follow-up was short. The much lower cost of off-label use of intravitreal bevacizumab is indisputable, but all three medications should be available to ophthalmologists who are responsible for tailoring the treatment for each patient with DMA.

\section{Ranibizumab}

Rationale. Ranibizumab (Lucentis) is a recombinant humanized Fab fragment of a monoclonal antibody, designed for intraocular use, which binds and inactivates all isoforms of VEGF-A. The rationale for anti-VEGF therapy for DME is based on the observation that VEGF levels are increased in the retina and vitreous of eyes with DR [74]. VEGF has been demonstrated to increase the vessel permeability in vivo by increasing the phosphorylation of tight junction proteins and thus is an important mediator of the BRB breakdown [75]. Therefore, therapy that inhibits VEGF represents an effective therapeutic modality targeting the underlying pathogenesis of DME. The intravitreal injection of ranibizumab has been shown to be beneficial and relatively safe for the treatment of DME in several randomized clinical trials and has become a standard for treatment of DME.

Evidence. Several studies reported the efficacy and superiority of ranibizumab to laser photocoagulation in the treatment of DME and long-term data recently became available, allowing the evaluation of continued therapy effects. The RESTORE study was the first study to demonstrate that ranibizumab monotherapy provides significantly superior benefit over laser in patients with visual impairment due to DME [24].

RESTORE was a phase III, randomized, doublemasked, 12-month study comparing ranibizumab monotherapy or combined with laser therapy with laser therapy alone [24]. Ranibizumab was given monthly during the first 3 months then PRN according to protocol-predefined retreatment criteria; laser was given at baseline as needed. The protocol required that monthly injections have to be continued if stable BCVA was not reached at the last two consecutive visits. At month 12, ranibizumab as monotherapy or combined with laser was significantly superior to laser monotherapy (mean BCVA gain $+6.1 /+5.9$ letters ranibizumab arms vs. +0.8 letters, laser monotherapy; 15 letter BCVA gain 22.6/22.9\% ranibizumab arms, vs. $8.2 \%$ laser monotherapy; Fig. 15). The mean number of sham treatments was similar in all treat- 


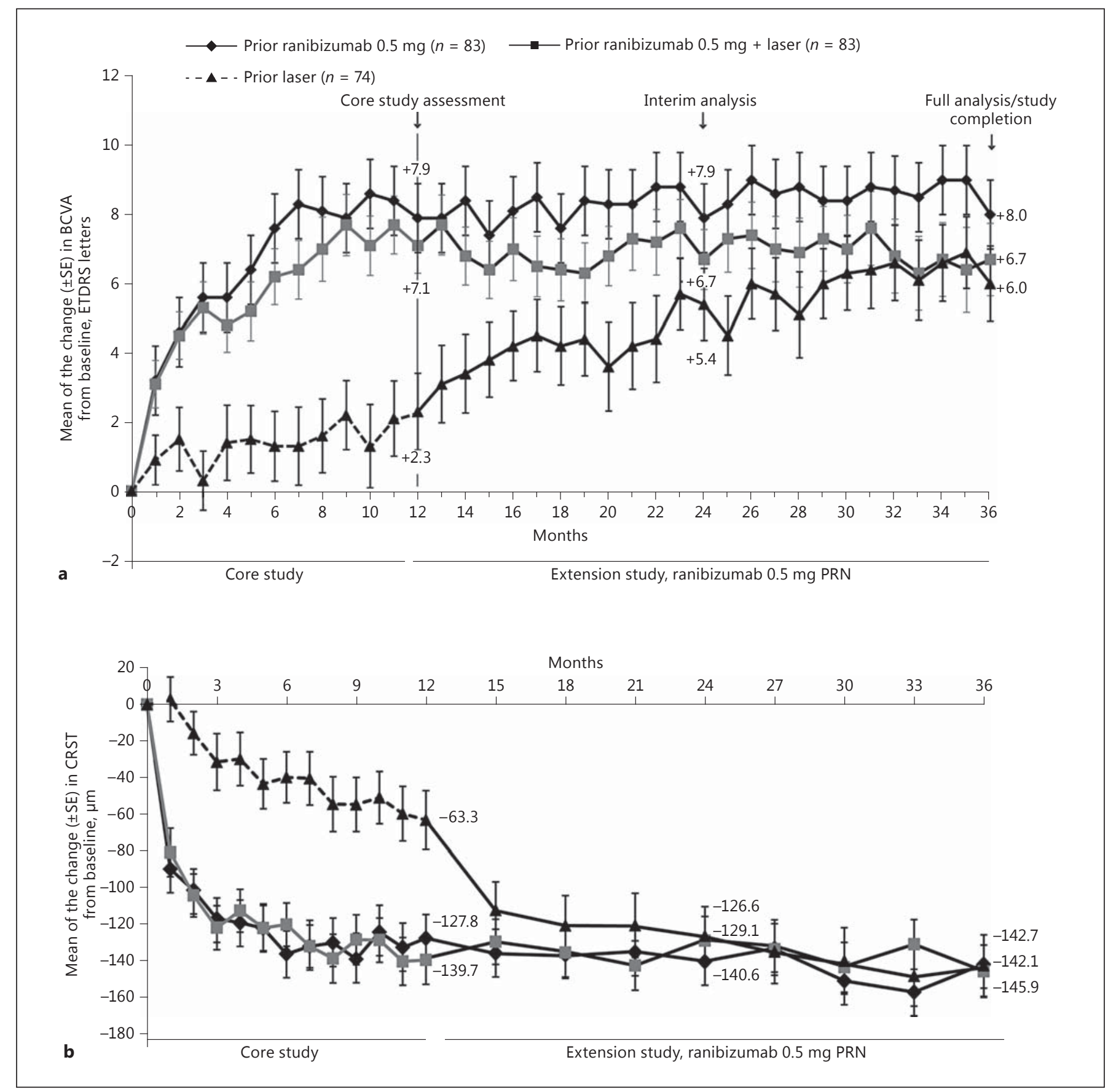

Fig. 16. RESTORE study. Mean of the change in best-corrected visual acuity (BCVA) letter score from baseline (day 1) to month 36 (a), and mean of the change in central retinal subfield thickness (CRST) score from baseline to month 36 (b) (safety set [last ob-

ment groups. The results of the RESTORE study demonstrate that treatment with ranibizumab is superior to laser treatment alone in improving BCVA in patients with visual impairment due to DME and that laser does not add

Guidelines for the Management of Diabetic Macular Edema by EURETINA servation carried forward]). ETDRS, Early Treatment Diabetic Retinopathy Study; PRN, pro re nata; SE, standard error. Reprinted from Schmidt-Erfurth et al. [26] with permission from Elsevier.

any benefit. During this 1-year study period, combining laser with ranibizumab did not seem to provide any advantage compared with ranibizumab monotherapy in terms of improving BCVA and treatment exposure.

Ophthalmologica 2017;237:185-222 DOI: $10.1159 / 000458539$ 
Table 1. RESTORE study: mean number of ranibizumab treatments received over 3 years (day 1 to month 35 , safety set)

\begin{tabular}{llll}
\hline Treatment period & \multicolumn{2}{l}{ Mean number of ranibizumab injections } & \\
\cline { 2 - 4 } & $\begin{array}{l}\text { prior ranibizumab } \\
0.5 \mathrm{mg}(n=83)\end{array}$ & $\begin{array}{l}\text { prior ranibizumab 0.5 } \mathrm{mg}+ \\
\text { laser }(n=83)\end{array}$ & $\begin{array}{l}\text { prior laser } \\
(n=74)\end{array}$ \\
\hline Day 1 to month 35 & 14.2 & 13.5 & $6.5^{\mathrm{a}}$ \\
Day 1 to month 11 & 7.4 & 7.5 & 0.0 \\
Months 12-23 & 3.9 & 3.5 & 4.1 \\
Months 24-35 & 2.9 & 2.5 & 2.4 \\
\hline
\end{tabular}

${ }^{a}$ Includes ranibizumab $0.5 \mathrm{mg}$ injections given over months $12-35$ only. Reprinted from Schmidt-Erfurth et al. [26] with permission from Elsevier.
Based on the results of the RESTORE study, ranibizumab was approved in Europe by the EMA for treatment of visual impairment due to DME in 2011. This approval was reviewed in 2014. The approved dose in Europe is 0.5 mg per injection compared to $0.3 \mathrm{mg}$ in the US. The medication is approved for monthly injections continued until the maximum visual acuity is achieved and/or the absence of activity of the disease.

The RESTORE Extension study followed the RESTORE patients until year 3. Patients with prior ranibizumab or combined therapy could maintain their prior CRT decreases and BCVA gains $(+8.0 /+6.7$ letters at year 3 ; Fig. 16) with a mean of only 3.7/2.7 retreatments per year (Table 1). Patients with prior laser therapy were able to receive ranibizumab PRN from year 2 onwards and achieved significant CRT decreases and BCVA gains (+6.0 letters; Fig. 16). A similar number of patients gained $>15$ letters until the end of year 3 across all 3 treatment arms, but a greater number of patients lost $>10$ letters until the end of year 3 in the prior laser arm (8.1 vs. 2.4\% [ranibizumab] $/ 4.8 \%$ [combined]). These results highlight the need of early therapy induction with ranibizumab to minimize the risk of significant BCVA loss, though the efficacy remains good in long-standing disease and therapy can be - if for any reason needed - begun at a later stage with a good chance for visual improvements [26].

These results are consistent with results from the DRCR.net Protocol I study, a multicenter randomized clinical trial showing that ranibizumab used in conjunction with laser therapy (prompt or deferred) was significantly more effective than laser alone in improving BCVA in patients with DME (BCVA gain +9 letters ranibizumab arms vs. +3 letters laser monotherapy at month 12 , mean number of injections $8-9$; >15 letter BCVA gain $30 \%$ ranibizumab pooled, $15 \%$ laser). In this study, complete BCVA maintenance from year 2 to year 3 with only a median of 1-2 injections could be achieved; this required a median number of monitoring visits of 7-8 in year 3. In years 4 and 5 of the study, the number of injections needed could be further reduced to $0-1$ injections (Table 2) while BCVA gains could be maintained [27, 47, 48, 67]. Refer to sections "Bevacizumab" and "Aflibercept" for results of the DRCR.net Protocol T study (Fig. 13 and 17) $[29,65]$. Noteworthy, the number of injections over 2 years in Protocol T was the same for all 3 drugs (aflibercept 15 [interquartile range 11-17], bevacizumab 16 interquartile range 12-20, and ranibizumab 15 [interquartile range 11-19] injections, respectively, global $p=0.08$ ). There was a decreasing number of injections for all 3 drugs in this study in the second year as well. The most important finding of Protocol T year 2 with regard to ranibizumab is that in patients with poorer baseline BCVA there is no more statistically significant difference between ranibizumab- and aflibercept-treated patients. Over a population level, ranibizumab could balance out the difference in the second year (Fig. 14) [65].

The RISE and RIDE studies were two parallel, methodologically identical, 2 year, phase III, multicenter, double-masked randomized studies comparing two dosing regimens of ranibizumab ( $0.3 \mathrm{vs.} 0.5 \mathrm{mg}$ ) as a monotherapy with fixed monthly injection regimens to laser monotherapy in the US [23]. In the two ranibizumab arms, the mean BCVA gain was +10.9-12.5 letters without a significant difference between the two dosing regimens (vs. laser $+2.6 /+2.3$ letters; $>15$-letter gain $18.1 / 12.3 \%$ in laser, $44.8 / 33.6$ and $39.2 / 45.7 \%$ in ranibizumab $0.3 / 0.5 \mathrm{mg}$ in RISE and RIDE; Fig. 18). Based on the results of the RISE and RIDE studies, ranibizumab was approved in the US by the FDA for treatment of DME in 2012. The approved dose is $0.3 \mathrm{mg}$ per injection. The medication is approved for monthly injections.

The 3-year outcomes of RISE and RIDE demonstrated that BCVA gains and improvement in retinal anatomy achieved with ranibizumab therapy at the end of year 2
202

Ophthalmologica 2017;237:185-222 DOI: $10.1159 / 000458539$
Schmidt-Erfurth et al. 
Table 2. DRCR.net Protocol I study: visits and treatments before 5-year visit

\begin{tabular}{lcc}
\hline & $\begin{array}{c}\text { Ranibizumab }+ \text { prompt } \\
\text { laser treatment }(n=124)\end{array}$ & $\begin{array}{l}\text { Ranibizumab }+ \\
\text { deferred laser } \\
\text { treatment }(n=11)\end{array}$ \\
& & \\
\hline Visit history, number of visits & $13(12-13)$ & $13(12-13)$ \\
Year 1 & $8(6-11)$ & $10(7-12)$ \\
Year 2 & $7(4-10)$ & $8(5-11)$ \\
Year 3 & $5(4-9)$ & $6(4-9)$ \\
Year 4 & $4(3-7)$ & $5(3-7)$ \\
Year 5 & $38(31-47)$ & $40(34-49)$ \\
Before 5-year visit & & $9(6-11)$ \\
Intravitreous injection history, number of injections & $8(7-11)$ & $3(1-6)$ \\
Year 1 & $2(0-5)$ & $2(0-5)$ \\
Year 2 & $1(0-4)$ & $1(0-4)$ \\
Year 3 & $0(0-3)$ & $0(0-3)$ \\
Year 4 & $0(0-3)$ & $61(55)$ \\
Year 5 & $57(46)$ & $53(48)$ \\
Eyes that received $\geq 1$ injections in year 4 & $47(38)$ & $17(11-27)$ \\
Eyes that received $\geq 1$ injections in year 5 & $13(9-24)$ & $0(0-2)$ \\
Before 5-year visit & $3(2-5)$ & $62(56)$ \\
Focal/grid laser history & & $108(97)$ \\
Focal/grid laser treatments before the 5-year visit & $0(0)$ & \\
Eyes that did not receive focal/grid laser treatment & $112(90)$ & \\
before the 5-year visit & & \\
Eyes that did not receive focal/grid laser treatment in & & \\
year 5 & & \\
\hline
\end{tabular}

Data are presented as median (quartiles) or $n(\%)$. Data are limited to study participants completing the 5-year visit. Reprinted from Elman et al. [48] with permission from Elsevier.

were sustained through year 3 without any significant difference in the two dosing regimens (Fig. 19). The study design allowed patients in the laser group to cross over and receive monthly $0.5 \mathrm{mg}$ ranibizumab in the third year. This delayed ranibizumab treatment did not result in the same extent of BCVA improvement observed in patients originally randomized to ranibizumab (BCVA gain $>15$ letters was $22.0 / 19.2 \%$ in the laser + delayed ranibizumab compared to $36.8-51.2 \%$ in the $0.3 / 0.5 \mathrm{mg}$ ranibizumab RISE/RIDE study arms). The mean BCVA gain from baseline to the end of year 3 was $+4.7 / 4.3$ letters in the laser + delayed ranibizumab group versus +10.614.2 in the ranibizumab arms of both studies, which indicates a permanent loss of BCVA if DME is treated too late with anti-VEGF [69]. An open-label extension of the studies allowed patients to be followed for another 14.1 months of ranibizumab PRN treatment. While maintaining 3-year BCVA, a mean of 4.5 injections were administered, and $25 \%$ of patients required no further injections [76].

Guidelines for the Management of Diabetic Macular Edema by EURETINA
In none of the studies were overall significant differences observed in BCVA gains between ranibizumab monotherapy and ranibizumab combined with laser treatment. The combined therapy was not superior to drug monotherapy and is therefore not recommended [77]. The RETAIN study, a multicenter controlled phase IIIB trial, evaluated the efficacy and safety of $0.5 \mathrm{mg}$ ranibizumab in 2 "treat and extend" treatment algorithms compared with a PRN regimen over 2 years. In "treat and extend," patients treated with a gradually prolonged time interval between injections after BCVA stabilization with monthly injections has been reached. The RETAIN study confirmed that this regimen is noninferior to PRN in mean average BCVA gain after 2 years $(+8.3 /+6.5$ vs. +8.1 letters [PRN]; Fig. 20) with a mean of $12.4 / 12.8$ versus 10.7 injections but with a large reduction in patient visits (9.0/8.9 vs. 16.6 visits) [30].

In all these studies as well as in a meta-analysis [77] of 1,500 patients treated with ranibizumab in randomized clinical trials, the safety profile of ranibizumab was excel-

Ophthalmologica 2017;237:185-222 203 
Fig. 17. DRCR.net Protocol T study. Mean change in central subfield thickness over time: shown are the changes in central subfield thickness overall (a) and according to baseline visual acuity (b). b Solid lines indicate baseline visual acuity of 20/50 or worse, and dashed lines indicate baseline visual acuity of 20/32 to 20/40. Central subfield thickness was assessed with the use of optical coherence tomography. The number of eyes assessed at each 4-week interval ranged from 192 to 221 in the aflibercept group, 186-216 in the bevacizumab group, and $185-215$ in the ranibizumab group. $T$ bars indicate $95 \%$ confidence intervals. Reprinted from Wells et al. [29] with permission from Massachusetts Medical Society.
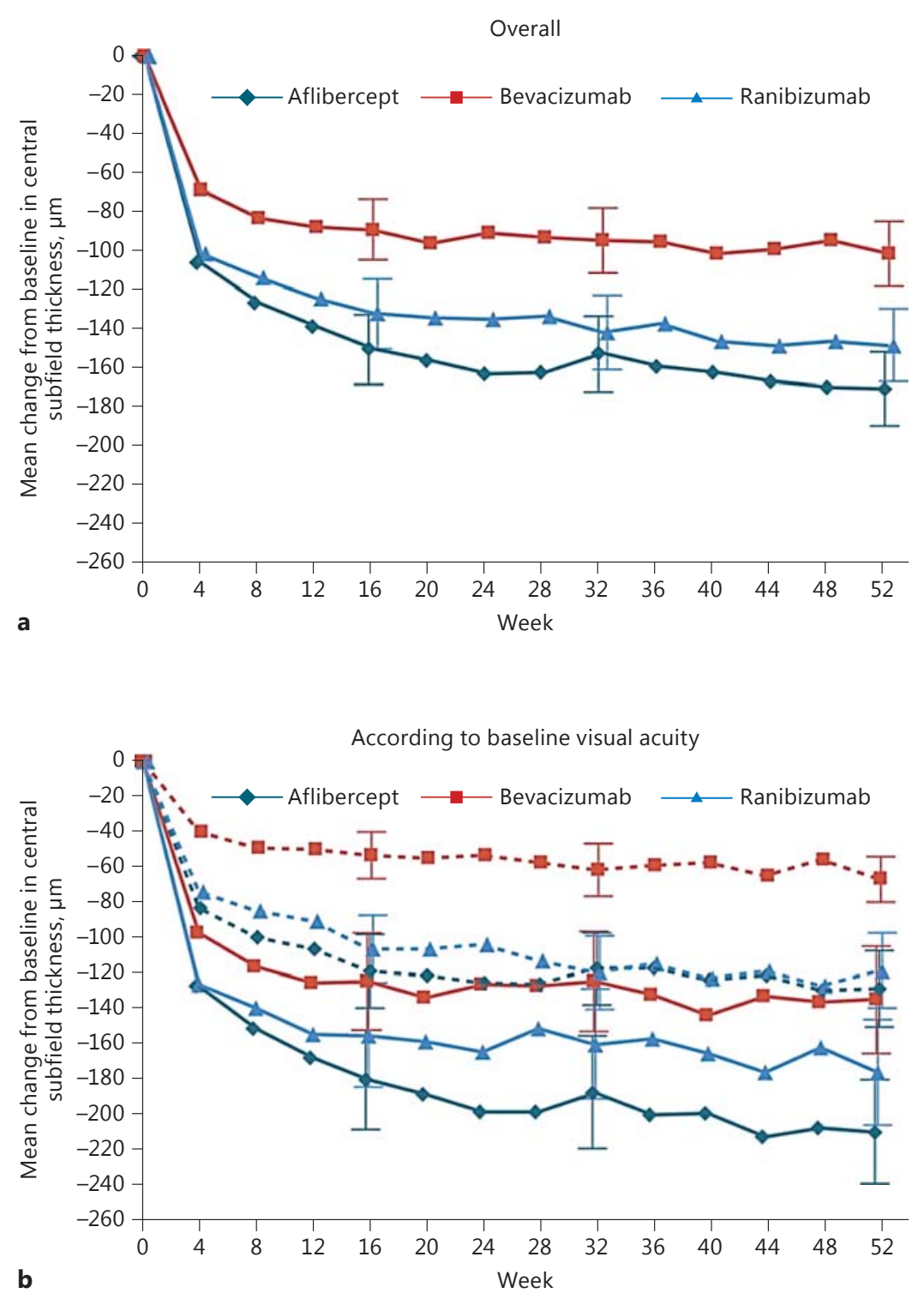

lent, without an increase of cardiovascular events compared to sham. No systemic safety concern emerged with 0.3/0.5 mg ranibizumab administered monthly compared with control through the RISE/RIDE extension study to 3 years [69]. In Protocol I, patients in the sham group experienced higher rates of Anti-Platelet Trialists' Collaboration (APTC)-classified systemic events than patients receiving ranibizumab [27]. In RESTORE, no meaningful differences in the number of arterial thromboembolic events or other systemic events were observed between the ranibizumab and the laser groups, although patients with a history of stroke or transient ischemic attack were primarily excluded from this study [24].

When looking at the different treatment regimens available for ranibizumab injection, studies are available for a fixed monthly injection [23], a PRN regimen [24], or a treat and extend regimen. Monthly injections seem to be feasible in the beginning of therapy, but are not required over a period of more than 3-6 months as confirmed by the decreasing numbers of injections needed after month 6 in several studies (Tables 1 and 2) $[25,26$, 48,69 ], even starting with a PRN regimen from baseline 


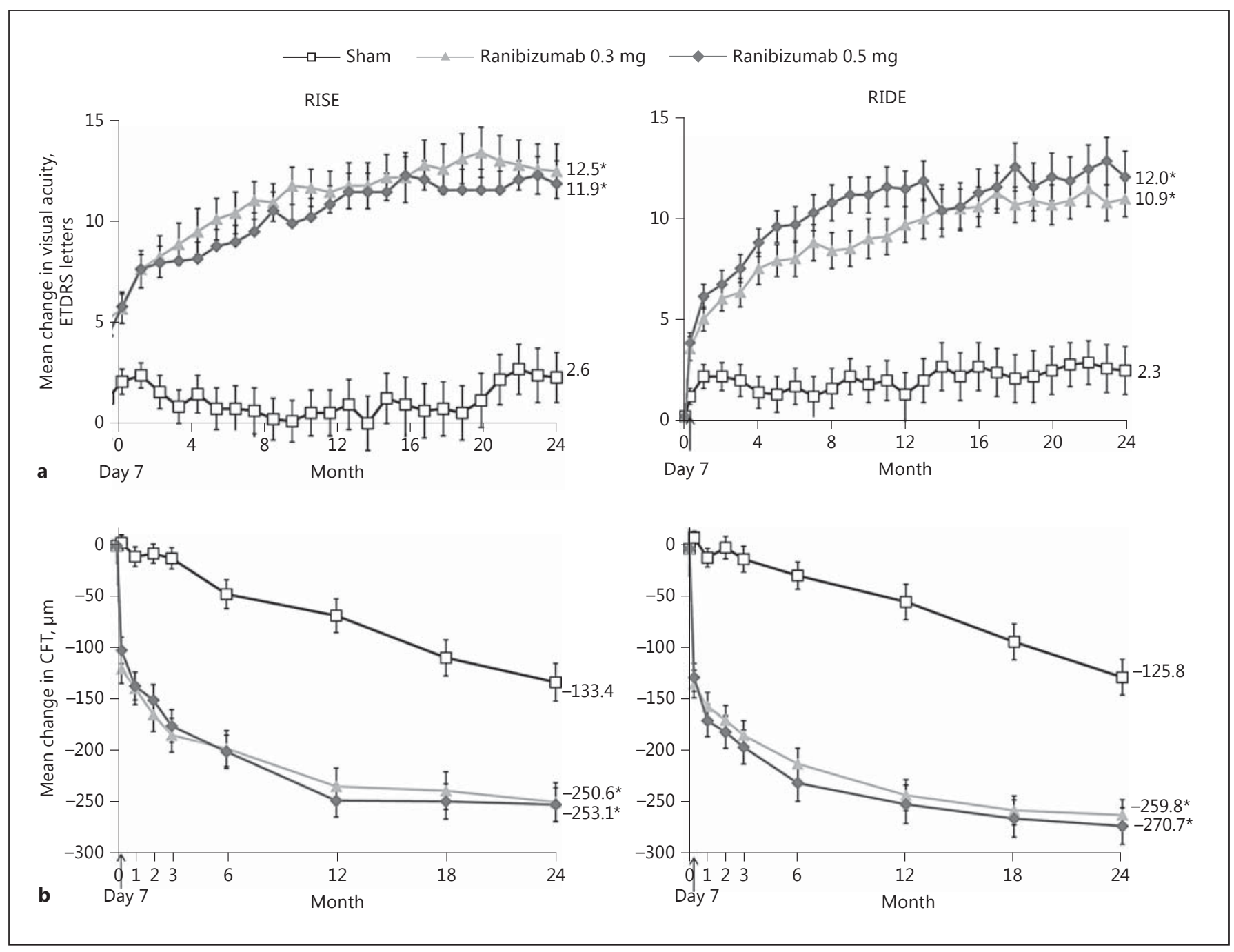

Fig. 18. RIDE/RISE studies. Changes in visual acuity (a) and central foveal thickness (CFT) (b) from baseline through 24 months. Number of patients: 127, 125, and 125 (RISE), and 130, 125, and 127 (RIDE) in the sham, $0.3 \mathrm{mg}$, and $0.5 \mathrm{mg}$ groups, respectively. Vertical bars are \pm 1 standard error of the mean. The last observation carried forward imputation method was used. ${ }^{*} p<0.0001$ vs. sham (analysis of variance $t$ test [stratified]). Differences were statistically significant starting at the first posttreatment observation (day 7) and at each point thereafter; a hierarchical testing strategy controlled for multiple comparisons. ETDRS, Early Treatment Diabetic Retinopathy Study. Reprinted from Nguyen et al. [23] with permission from Elsevier. results in comparable BCVA gains [29]. Retreatment and monitoring intervals can be adjusted to visual acuity or anatomic response to ranibizumab. Most studies propose monthly monitoring, but in certain cases bimonthly monitoring, as done in the RELIGHT study [78], can be applied to reduce patient burden and monitoring costs. None of the proposed retreatment criteria in a PRN regimen can be considered to be superior to the others as no comparability study has been performed. An alternative approach is the "treat and extend regimen." The largest

Guidelines for the Management of Diabetic Macular Edema by EURETINA criticism about this regimen in the long-term is the fact that each time treatment has to be administered even if disease and vision stability is already achieved, even if there are no concerns about efficacy and safety in 2 years of this retreatment regimen, there might be overtreatment, especially as DME seems to plateau after the first treatment period and very little or no injections are needed, which has been referred to as a disease-modifying effect [48]. Bimonthly monitoring and "treat and extend" suggest that a flexible retreatment regimen of ranibizum- 


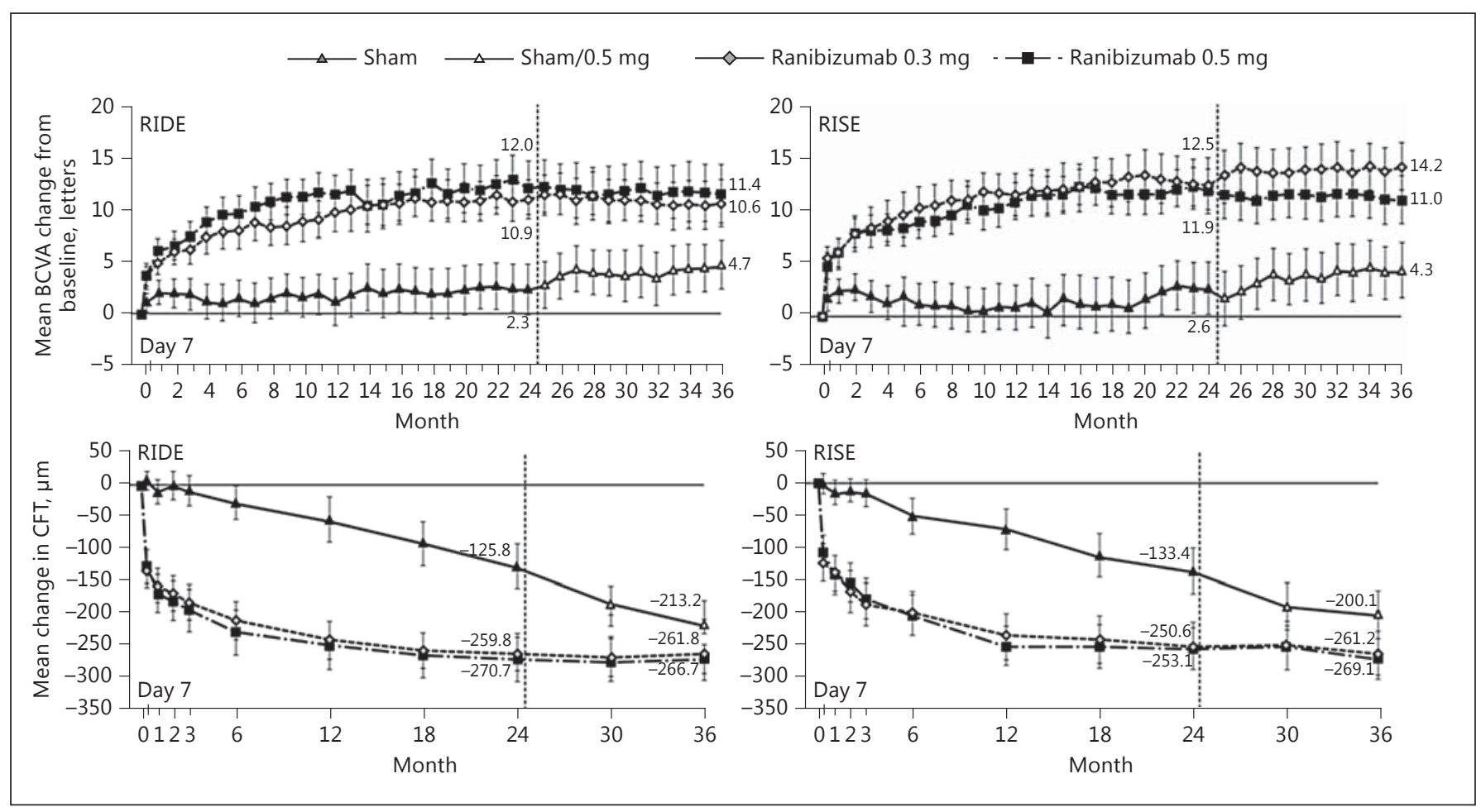

Fig. 19. RIDE/RISE studies. Mean change in best-corrected visual acuity (BCVA) and central foveal thickness (CFT) from baseline over time. Vertical bars are 95\% confidence intervals. Missing data were imputed by last observation carried forward. Reprinted from Brown et al. [69] with permission from Elsevier.

ab with extended intervals between visits may be a viable strategy for the management of most patients with DME.

Recommendation. Ranibizumab can be used equivalent to other available anti-VEGF agents for patients with a baseline BCVA letter score of 69 letters and above. The use of ranibizumab for patients with poorer baseline visual acuity will most likely result in the same visual acuity results after 2 years of treatment as aflibercept, but the effect will be reached slightly slower. Therefore, treatment shall be initiated with aflibercept, if available, in these patients. It remains unclear to which extent the slower effect of ranibizumab seen in Protocol T compared to aflibercept can be accounted to the lower dose $(0.3 \mathrm{mg})$ of ranibizumab used in this study.

Treatment with ranibizumab shall be initiated early on with monthly injections. If visual acuity improves and/or CRT decreases or other morphological signs for disease activity can be found, monthly injections must be continued until visual acuity and/or OCT stability is reached. Thereafter, patients should in the best case be monitored monthly in the first year with visual acuity testing and OCT imaging. Then, the interval of injections and moni- toring visits can be extended upon visual acuity and/or anatomic stability. If no more functional or anatomical benefit occurs, the treatment must be stopped, and extended monitoring intervals can be evaluated for each patient individually.

\section{Aflibercept}

Rationale. Aflibercept (Eylea) is a recombinant decoyreceptor type of inhibitor of VEGF and placental growth factor that has shown efficacy and safety in adequate clinical trials in DMA $[28,29]$.

Evidence. The evidence for the value of DME treatment by intravitreal injections of aflibercept is dominated by the twin pair of randomized controlled clinical studies VIVID DME (Europe) and VISTA DME (US) [28, 79], with a total of 872 study participants, which compared aflibercept with laser therapy, and the DRCR.net Protocol T study [29], with a total of 660 participants, which compared intravitreal aflibercept with ranibizumab and bevacizumab.

The VIVID-VISTA DME studies had their primary endpoint at 1 year and were planned to follow patients up 


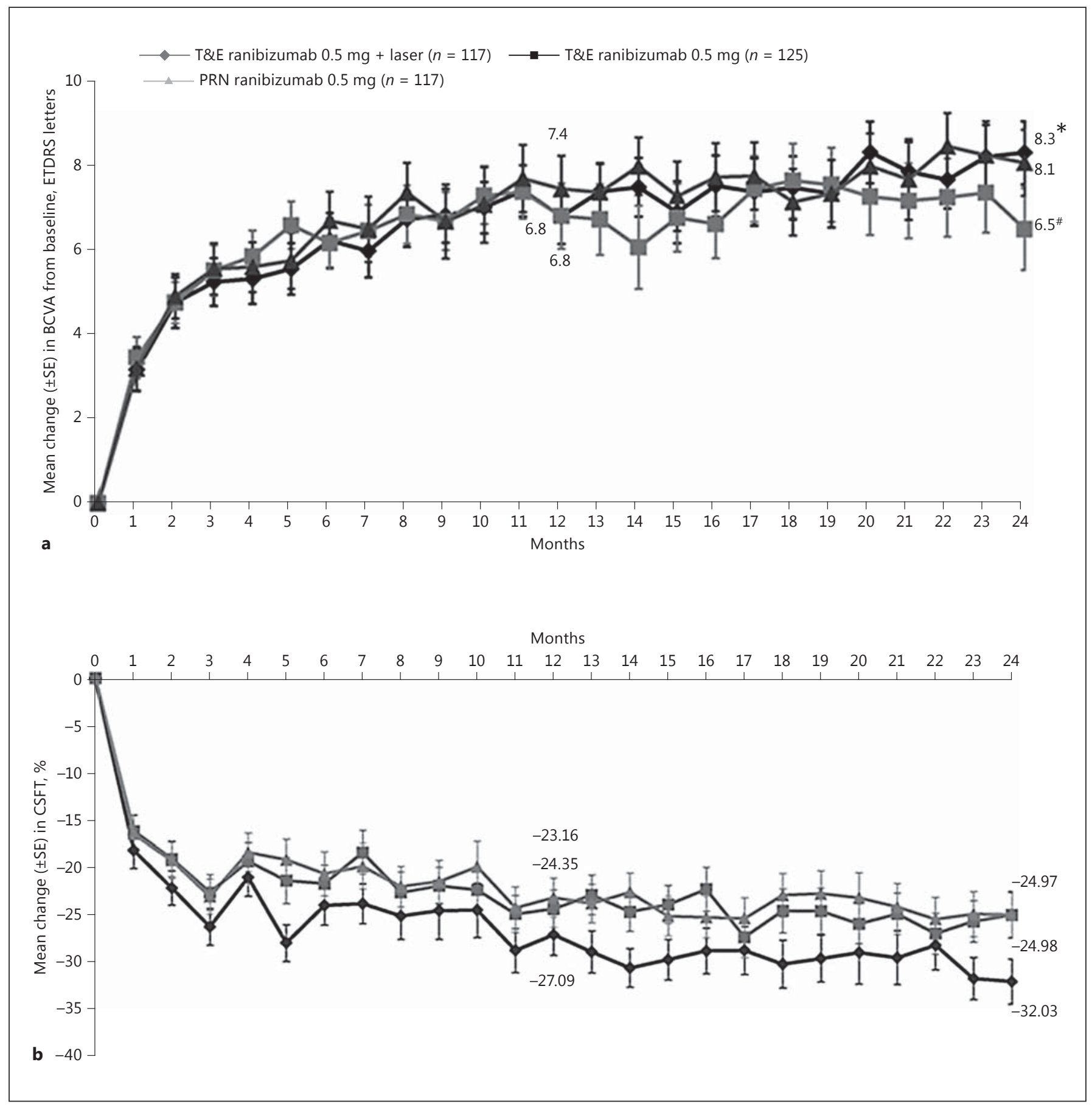

Fig. 20. RETAIN study. a Mean change in best-corrected visual acuity (BCVA) from baseline to months 12 and 24 (full analysis set [FAS] - mean value imputation/last observation carried forward $[\mathrm{MV} / \mathrm{LOCF}]) .{ }^{*} p=0.9327$ vs. pro re nata $(\mathrm{PRN}) ;{ }^{*} p=0.1599 \mathrm{vs}$. PRN; Cochran-Mantel-Haenszel test (row mean scores statistic) with the observed values as scores. b Mean percentage change in central subfield thickness (CSFT) from baseline over time (FAS$\mathrm{MV} / \mathrm{LOCF}$ ). a, b FAS (MV/LOCF) comprised all randomized pa- tients who received at least one application of study treatment (ranibizumab or laser) and had at least one postbaseline efficacy assessment in the study eye. Stratified analysis included baseline visual acuity ( $\leq 60$ letters, $>60$ and $\leq 73$ letters, and $>73$ letters) as factors. ETDRS, Early Treatment Diabetic Retinopathy Study. Reproduced from Prünte et al. [30] with permission from BJM Publishing Group Ltd.
Guidelines for the Management of Diabetic Macular Edema by EURETINA
Ophthalmologica 2017;237:185-222 DOI: $10.1159 / 000458539$ 
to 3 years. Laser or aflibercept were administered in eyes with DME involving the foveal center with rescue therapy, consisting of laser treatment, permitted after 24 weeks. Aflibercept $2 \mathrm{mg}$ was given every 4 weeks or every 8 weeks after 5 initial monthly loading doses. The studies enrolled patients with a BCVA letter score of 24-73 ETDRS letters and an OCT central subfield thickness $\geq 300 \mu \mathrm{m}$.

The mean BCVA gain at 1 year for the combined studies was 11.6 BCVA letters for 4-weekly injections, and it remained stable at 10.7 letters after 100 weeks. For 8-weekly injections, BCVA had similarly increased by 10.7 letters after 52 weeks and was also stable after 100 weeks with a gain of 10.3 letters. These clinically and statistically insignificant differences suggest that the 8-weekly interval regimen should be preferred, if a fixed regimen is chosen.

The outcome in eyes that had previously received antiVEGF therapy was comparable to the outcome in treatment-naïve eyes. After 100 weeks, 31.2 and $24.1 \%$ of eyes initially treated with laser in VIVID and VISTA, respectively, received aflibercept therapy and BCVA gradually increased, but a mean deficit of 5-6 letters persisted after 100 weeks highlighting the benefit of timely treatment initiation.

A conspicuous finding in VIVID-VISTA DME was that the switch to bimonthly injections after 24 weeks was followed by an oscillating pattern of CRT variation with an amplitude of $25-50 \mu \mathrm{m}$ and period of 8 weeks. There was no evidence that this fluctuation was associated with a poorer BCVA outcome overall compared to eyes receiving injections every 4 weeks throughout the first 100 weeks of the study. Based on the results of the VIVIDVISTA DME studies, aflibercept was approved in Europe (2014) by the EMA and in the US (in 2015) by the FDA for treatment of DME. The approved dose is $2 \mathrm{mg}$ per injection. The medication was approved for 5 monthly injections followed by bimonthly injections in the US, and the European label adds the possibility of extending the treatment interval after the first year of treatment.

The DRCR.net Protocol T study [29] (refer to the section "Bevacizumab" for full study results) compared aflibercept, ranibizumab, and bevacizumab. The loading phase and subsequent flexible retreatment phase regimen was the same for all 3 study drugs. The study enrolled eyes with BCVA letter scores of 24-78 (approx. Snellen 20/320 to 20/32) and DME involving the foveal center with CRT greater than roughly $325 \mu \mathrm{m}$ on modern SD-OCT $(>250$ $\mu \mathrm{m}$ on Zeiss Stratus with gender-specific cutoffs) [80]. The interim results after 1 year showed a mean gain that was +2.1 letters higher for aflibercept $2 \mathrm{mg}$ than for ranibizumab $0.3 \mathrm{mg}$ (the approved dose in the US, $0.5 \mathrm{mg}$ is the approved dose in Europe) $(p=0.03)$. Patients were monitored as often as every 4 weeks. A subgroup analysis showed that the superior effect of aflibercept was driven by the study participants with poorer baseline BCVA (<69 letters). Of a maximum possible number of injections of 13 in the first year, the aflibercept arm received a median of 9 injections; the bevacizumab and ranibizum$\mathrm{ab}$ arms received a median of 10 injections. Intravitreal bevacizumab was inferior to both aflibercept and ranibizumab in most comparisons. Serious adverse event rates were comparable between study arms.

The 2-year results [65] of the Protocol T study slightly changed this scenario. The difference in BCVA gain between aflibercept and ranibizumab for eyes with poorer baseline BCVA that was noted at 1 year decreased at 2 years. Nevertheless, the first-year behavior and the slightly better mean BCVA gain confirmed the superiority of aflibercept over ranibizumab in patients with poorer baseline BCVA (Fig. 14) when considering the area under the curve. It remains unclear if the $0.5 \mathrm{mg}$ dose that is approved in Europe would have led to different results in the first year of Protocol T in favor of ranibizumab $0.5 \mathrm{mg}$.

Longer follow-up is needed to determine the rate at which aflibercept treatment of DME can be tapered after 1 year and whether long-term aflibercept treatment is associated with a diminishing need for reinjection, as in the case of ranibizumab in the 5-year follow-up of the DRCR. net Protocol I study [48]. At least the 2-year results of Protocol T suggest that the need for anti-VEGF injection generally decreases in DME from year to year. In Protocol $\mathrm{T}$, for all anti-VEGF agents about half of the injections needed in the first year were needed in the second year of the study.

Recommendation. Aflibercept has shown clear superiority to laser therapy in visual and anatomical outcomes. It can be used equivalent for improving vision in eyes with a baseline BCVA letter score of 69 or more. Aflibercept is the drug of choice in DME eyes with baseline BCVA below 69 letters, as it shows superiority to bevacizumab over 2 years and over ranibizumab in the first year of treatment.

The evidence base for the use of aflibercept in DME leaves it an open choice as to whether a course of loading injections at 4-weekly intervals should be followed by a regimen of fixed bimonthly injections or a PRN regimen with monthly monitoring only. The gain of 13.3 letters at 1 year in Protocol T with $2.0 \mathrm{mg}$ aflibercept PRN and the gain of 10.7 letters with $2 \mathrm{mg}$ aflibercept bimonthly after a loading phase of 5 monthly injections in VIVID-VISTA DME were obtained in different trials and a direct com-
208

Ophthalmologica 2017;237:185-222 DOI: $10.1159 / 000458539$
Schmidt-Erfurth et al. 
parison of the 2 regimens has not been made. Intravitreal aflibercept offers most patients with visual loss secondary to DME that involves the foveal center a chance that their visual acuity will significantly improve after treatment and fewer injections might be needed from the second year onwards.

\section{Steroids}

Rationale

Recent evidence highlights to the role of inflammation in the development of DME. The pathological processes include leukostasis, which is the accumulation of leukocytes on the surface of retinal capillaries and is thought to be a major player and early event in the dysfunction of the BRB [81]. Such leukostasis leads to the upregulation of intracellular adhesion molecule (ICAM)-1, which mediates attraction of monocytes and neutrophils to the vascular endothelium. ICAM-1 has been found to further enhance retinal leukostasis, vascular permeability, and breakdown of the BRB in diabetes [82].

After binding to the vascular endothelium, leukocytes produce reactive oxygen species and inflammatory cytokines, which leads to increased vascular permeability [83]. Animal experiments showed that elevated glucose levels in the blood serum may lead to the expression of interleukin-6 (IL-6), tumor necrosis factor, lymphotoxin, and cyclooxygenase-2 [84]. IL-6 and pigment epitheliumderived factor expression have been found to be significantly increased in the vitreous of diabetic eyes [85].

Corticosteroids produce an anti-inflammatory effect through various mechanisms, including the decrease in the synthesis of inflammatory mediators, as well as the decrease in VEGF synthesis [86]. Intravitreal treatment with glucocorticoids has been found to significantly decrease the inflammatory processes with improved $\mathrm{BRB}$ function through inhibition of ICAM-1 expression in rat retinas [87]. A study that evaluated the changes in aqueous levels of inflammatory (IL-6, IL-8, interferon-induced protein-10, monocyte-chemoattractant protein-1, platelet-derived growth factor [PDGF]-AA) and angiogenic (VEGF) cytokines after intravitreal injection of corticosteroid triamcinolone in comparison to the anti-VEGF agent bevacizumab in patients with DME, found that IL-6, inducible-protein 10, monocyte-chemoattractant protein-1, PDGF-AA and VEGF were significantly decreased in triamcinolone-treated eyes, but only VEGF levels were decreased in the eyes treated with bevacizumab [88]. Therefore, their use in the treatment of DME may be more comprehensive than anti-VEGF treatment, which targets only a part of the inflammatory cascade [89].

Guidelines for the Management of Diabetic Macular Edema by EURETINA
Evidence

Commercially available corticosteroid compounds for intravitreal use include triamcinolone acetonide, dexamethasone posterior segment delivery system (Ozurdex), and fluocinolone (Iluvien).

Triamcinolone Acetonide. The DRCR.net protocol B was an important milestone in evaluating triamcinolone's role in DME management. It was a multicenter, randomized clinical trial with a total of 840 study eyes of $693 \mathrm{sub}$ jects with DME involving the fovea and BCVA of 20/40 to $20 / 320$. Patients were randomized to focal/grid laser therapy, $1 \mathrm{mg}$ intravitreal triamcinolone, or $4 \mathrm{~g}$ intravitreal triamcinolone. Retreatment was given at 4-month intervals. While at 4 months, the mean BCVA was better in the $4 \mathrm{mg}$ group than in the laser or $1 \mathrm{mg}$ groups, by the end of the first year there were no significant differences among the groups with regard to mean BCVA. At the 16 -month visit and extending through 2 years, mean BCVA was better in the laser group than in the 2 triamcinolone groups. The majority of patients in the triamcinolone groups had developed cataract by this time, but the differences could not be attributed solely to cataract formation. More eyes in the $4 \mathrm{mg}$ group (40\%) than in the $1 \mathrm{mg}$ group (20\%) or laser group (10\%) suffered from an ocular hypertension-related adverse event, including either an elevation of intraocular pressure (IOP) of $\geq 10$ $\mathrm{mm} \mathrm{Hg}$ from baseline, an IOP of $\geq 30 \mathrm{~mm} \mathrm{Hg}$, an initiation of IOP-lowering medications or the diagnosis of glaucoma. Glaucoma surgery was performed in 4 eyes in the $4 \mathrm{mg}$ group. Among phakic eyes at baseline, cataract surgery was performed by the end of the second year in $23 \%$ of eyes in the $4 / 1 \mathrm{mg}$ groups vs. $13 \%$ only in the laser group [90].

Another important milestone for triamcinolone acetonide was the DRCR.net protocol I study. As mentioned earlier, this was a 5-year, independent, multicenter, randomized, controlled clinical trial. A total of 854 eyes of 691 participants with BCVA of 20/32 to 20/320 and DME involving the fovea were randomized to sham injection + prompt laser, $0.5 \mathrm{mg}$ ranibizumab + prompt laser, $0.5 \mathrm{mg}$ ranibizumab + deferred ( $\geq 24$ weeks) laser, or $4 \mathrm{mg}$ triamcinolone acetonide + prompt laser. At 1 year, treatment with triamcinolone and laser resulted in a gain of 4 letters from baseline compared with a 3-letter gain in the laser group, and a 9-letter gain in both the ranibizumab and laser groups. In a subgroup of pseudophakic patients treated with triamcinolone and laser, BCVA gain was comparable to that of pseudophakic eyes treated with ranibizumab and superior to that of pseudophakic eyes treated with laser only. More eyes in the triamcinolone

Ophthalmologica 2017;237:185-222 DOI: $10.1159 / 000458539$ 
group (50\%) than in the ranibizumab groups (9\%) or the laser group (11\%) had an IOP elevation $\geq 10 \mathrm{~mm} \mathrm{Hg}$, IOP $>30 \mathrm{~mm} \mathrm{Hg}$ or initiation of IOP-lowering medications during 2 years of follow-up. Glaucoma surgery was performed in 4 eyes (one eye in the laser group, one eye in the ranibizumab + prompt laser group, and two eyes in the triamcinolone group). More eyes in the triamcinolone group (59\%) underwent cataract surgery over two years of follow-up than in the laser group (14\%) or the ranibizumab groups (14\%) [27].

Dexamethasone. The first study that evaluated dexamethasone for DME was the PLACID trial. The study was a randomized, controlled, multicenter clinical trial. A total of 253 patients with center involving DME, with a BCVA letter score of 34-70 letters and CRT $\geq 275 \mu \mathrm{m}$ were randomized to $0.7 \mathrm{mg}$ Ozurdex implant and laser therapy at month 1 or laser monotherapy with a sham implant injection. Subjects could receive up to 3 additional laser treatments and 1 additional Ozurdex injection. While at 1 year there was no difference between the groups, the percentage of patients gaining at least 10 letters was significantly higher in the Ozurdex plus laser group vs. the laser monotherapy group at month 1 (31.7 vs. $11 \%, p<0.001)$ and month 9 (31.7 vs. $17.3 \%, p=$ $0.007)$. Increased IOP occurred more frequently in the Ozurdex plus laser group than in the laser monotherapy group, with $15.2 \%$ of patients experiencing an increase of $\geq 10 \mathrm{~mm} \mathrm{Hg}$ from baseline, $16.8 \%$ experiencing an IOP of $\geq 25 \mathrm{~mm} \mathrm{Hg}$ and $4 \%$ an IOP of $\geq 35 \mathrm{~mm} \mathrm{Hg}$. In $15.9 \%$ of patients in the Ozurdex plus laser group vs. $1.6 \%$ patients in the laser monotherapy group treatment with IOP-lowering medication was begun. However, no eyes required surgery to control IOP. Cataract-related adverse events were more common among phakic patients in the Ozurdex plus laser group (22.2\%) versus the laser monotherapy group $(9.5 \%)$. Cataract surgery was performed in 4 eyes in the Ozurdex plus laser group in comparison to 5 eyes in the laser monotherapy group [91].

The most important trial evaluating Ozurdex for DME were the MEAD trials. MEAD were 2 randomized, multicenter, masked, sham-controlled, phase III trials with identical protocols. A total of 1,048 participants with central DME, BCVA of 20/50 to 20/200 and CRT of $\geq 300 \mu \mathrm{m}$ were randomized to treatment with Ozurdex $0.7 \mathrm{mg}$, Ozurdex $0.35 \mathrm{mg}$ or a sham procedure with a follow-up of 3 years. Retreatment was allowed according to predefined retreatment criteria, not more frequently than every 6 months. At the end of 3 years, the percentage of patients with a $\geq 15$-letter gain of BCVA from baseline was $22.2 / 18.4 \%$ in the Ozurdex $0.7 / 0.35 \mathrm{mg}$ group versus $12 \%$ in the sham group. An IOP increase of $\geq 10 \mathrm{~mm} \mathrm{Hg}$ occurred in $27.7 / 24.8 \%$ of patients in the $0.7 / 0.35 \mathrm{mg}$ Ozurdex groups versus $13 \%$ in the sham group. An IOP of $\geq 35$ $\mathrm{mm} \mathrm{Hg}$ occurred in 6.6/5.2\% of patients in the Ozurdex $0.7 / 0.35 \mathrm{mg}$ groups versus $0.9 \%$ in the sham group. $41.5 / 37.6 \%$ of patients in the $0.7 / 0.35 \mathrm{mg}$ Ozurdex groups needed IOP-lowering medications versus $9.1 \%$ in the sham group. One patient in each Ozurdex treatment group underwent glaucoma incisional surgery. The rate of cataract-related adverse events was $67.9 / 64.1 \%$ in the $0.7 / 0.35 \mathrm{mg}$ Ozurdex groups, respectively, versus $20.4 \%$ in the sham group [92].

The CHAMPLAIN study was a prospective, multicenter, open-label, 26-week study. Fifty-five patients with treatment-refractory DME and a history of previous pars plana vitrectomy (PPV) in the study eye received a single intravitreal injection of $0.7 \mathrm{mg}$ Ozurdex. The mean BCVA gain from baseline was 6 letters after 8 weeks and 3 letters after 26 weeks. At 8 weeks, $30.4 \%$ of patients gained $\geq 10$ letters. Increased IOP was reported in $16 \%$ of study eyes, with no patients requiring a surgical procedure to control IOP. Cataract progression was reported in $17 \%$ of phakic eyes, with 1 patient undergoing cataract surgery [93].

Ozurdex $0.7 \mathrm{mg}$ was approved by the FDA in September 2014 for the treatment of adult patients with DME. It was approved by the EMA in July 2014 for the treatment of adult patients with visual impairment due to DME who are pseudophakic or who are considered insufficiently responsive to or unsuitable for noncorticosteroid therapy. The implant releases the corticosteroid into the vitreous over a period of $\leq 6$ months [94]. Therefore, the official product label in Europe recommends retreatment after approximately 6 months, and does not recommend administration to both eyes concurrently. Newer data support the reinjection of the dexamethasone implant earlier than the recommended retreatment interval. The CHROME study, a retrospective real-world study, included patients with DME, retinal vein occlusion, and uveitis. The mean reinjection interval in this study was 2.3-4.9 months [95].

Fluocinolone Acetonide. The FAME trials were 2 parallel, prospective, randomized, phase III, multicenter trials. A total of 956 patients with central DME, BCVA of 20/50 to $20 / 200$ and CRT $\geq 250 \mu \mathrm{m}$ were randomized to receive an intravitreal insert releasing $0.2 \mathrm{or} 0.5 \mu \mathrm{g}$ fluocinolone acetonide per day or sham injection. Based on predefined retreatment criteria, additional treatment could be given after 1 year. The percentage of patients with a BCVA gain of $\geq 15$ letters after 2 years was $28 \%$ in each of the fluocinolone groups versus $16 \%$ in the sham group [96].
210

Ophthalmologica 2017;237:185-222 DOI: 10.1159/000458539
Schmidt-Erfurth et al. 
However, in a subgroup analysis comparing chronic ( $\geq 3$ years from diagnosis) with acute DME, the percentage of patients who gained $\geq 15$ letters was significantly greater in chronic DME patients (34 vs. $13.4 \%$ in the sham group) compared to acute DME (22.3 vs $27.8 \%$ in the sham group). The rate of glaucoma requiring incisional surgery in both treatment groups was as high as $7.6 / 3.7 \%$ in the $0.5 / 0.2 \mu$ g groups versus $0.5 \%$ in the sham group. Cataract surgery was needed in $50.9 / 41.1 \%$ of patients in the $0.5 / 0.2$ $\mu \mathrm{g}$ groups versus only $7 \%$ in the sham group [97].

Iluvien has been approved by the FDA in September 2014 for the treatment of DME in patients who have been previously treated with a course of corticosteroids and did not have a clinically significant elevation of IOP. It was approved by the EMA in April 2014 for the treatment of vision impairment associated with chronic DME considered insufficiently responsive to other available therapies. The approved dose for the fluocinolone acetonide is $0.19 \mathrm{mg}$. Pharmacokinetic studies showed that it provides sustained delivery in the eye for at least one year [98]. Therefore, repeated treatment may be given after a year according to evidence of central fluid and visual acuity parameters. The official product label in Europe recommends additional treatments after one year and does not recommend administration to both eyes concurrently.

\section{Recommendation}

Based on the data that exist thus far, corticosteroids are important in our armamentarium of drugs for treating DME patients, but largely on a second choice level. In nonresponders who have already been treated with antiVEGF (after 3-6 injections, depending on the specific response of each patient), it is reasonable to switch to a steroid.

As first-line therapy, the use of steroids may be considered in patients who have a history of a major cardiovascular event as these patients were excluded from all major anti-VEGF trials. A meta-analysis assessing the risk in anti-VEGF-treated DME patients has shown that highrisk patients who received monthly anti-VEGF treatment over the course of two years possibly have an increased risk for death and potentially for cerebrovascular accidents [99]. The authors conclude that the cumulative exposure to anti-VEGF might be an independent risk factor. Especially in aforementioned patients, steroids might be the better option. Another group of patients in whom corticosteroids may be considered as first-line therapy are patients who are not willing to come for monthly injections (and/or monitoring) in the first 6 months of therapy. However, these patients' IOP still needs to be moni-

Guidelines for the Management of Diabetic Macular Edema by EURETINA tored. Dexamethasone shall be used first; fluocinolone may be appropriate for nonsteroid responders with chronic macular edema that is not responsive to other treatments. Since triamcinolone is not approved and causes more increase in IOP and cataract, it should be used only in patients who cannot get the approved agents for this indication.

Pseudophakic patients are preferred for the use of steroids, otherwise patients have to be informed about the high risk for cataract surgery. The IOP has to be monitored frequently in all cases. Retreatment can be considered after around 6 months (dexamethasone)/year (fluocinolone acetonide) if there is still evidence of residual edema and impaired vision.

\section{Surgical Therapy}

Rationale

The particular characteristics of the vitreous in diabetic patients may be related to the development and maintenance of macular edema; therefore, PPV has been suggested as a potential treatment option for DME. The advantages of PPV seem clear in DME patients with associated vitreoretinal traction, but the procedure remains controversial in nontractional cases. The secondary retinal changes and presence of a glistening, taut membrane in patients with traction can be assessed by biomicroscopy. However, OCT enables better evaluation of the vitreoretinal interface, clearly showing the presence of traction and posterior hyaloid abnormalities, such as thickening and partial detachment [100]. A posterior vitreous detachment is associated with a lower incidence of DME in patients older than 60 years [101, 102]; consistently, spontaneous development of vitreomacular separation is associated with higher DME cure rates and improvements in BCVA [103]. The effect of PPV on nontractional DME remains uncertain, although several theories have been proposed: removal of pathological vitreous and subclinical traction at the macula, elimination of inflammatory particles that could increase vessel permeability [104], improvements in oxygen concentration in the vitreous cavity, and retinal vessel changes with normalization of the macular blood flow and decreased leakage $[105,106]$. The internal limiting membrane (ILM) is closely related to Müller cells [107], and ILM removal has been proposed in DME to eliminate all tractions, vitreous remnants, and inflammatory factors [108].

Evidence

DME patients can be divided according to the presence/absence of traction. PPV surgery to treat DME was 
Fig. 21. SD-OCT showing anterior-posterior traction, neurosensory retinal detachment, and external retinal edema in a patient with DME (upper row) and no traction and edema resolution after PPV with ILM peeling and laser therapy (lower row). Images are courtesy of Pepe Garcia Arumi and Sergio Copete Piqueras.
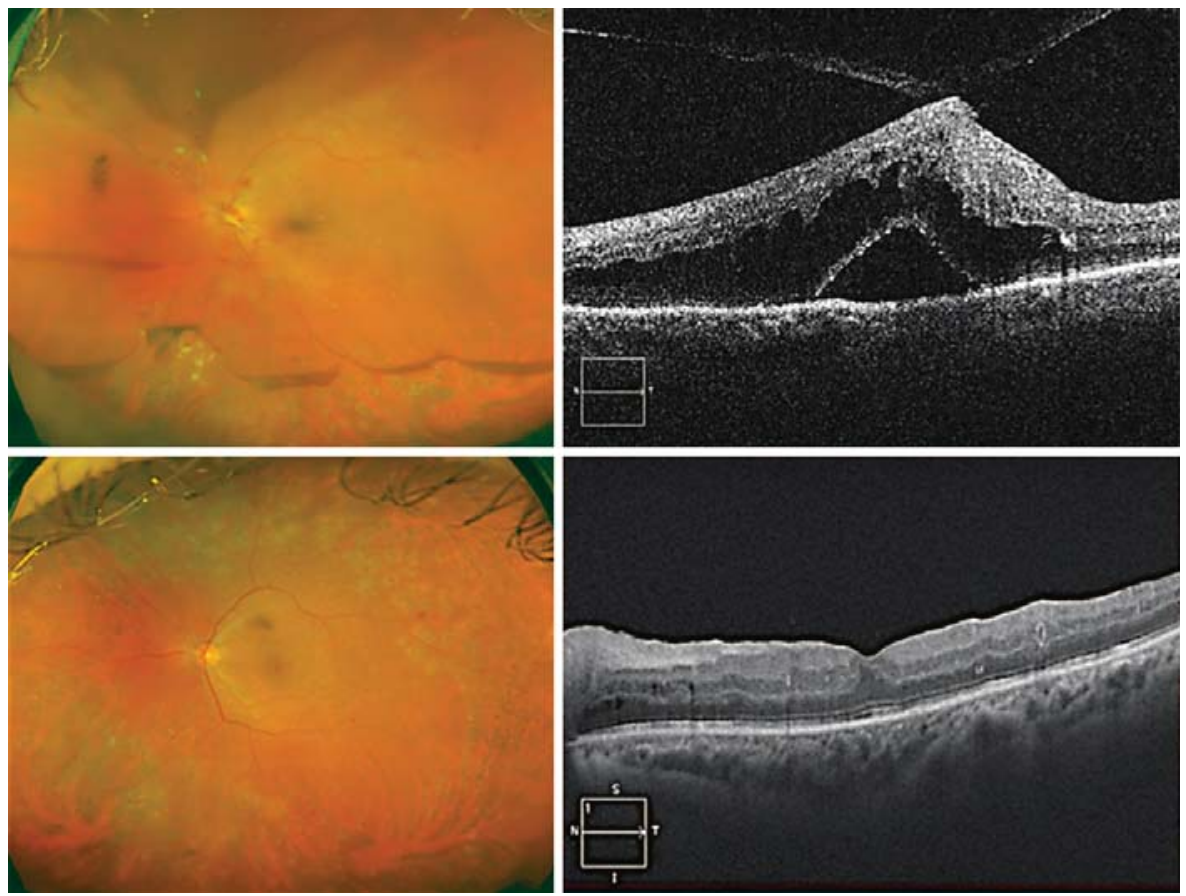

first described by Lewis et al. [109] in 1992. PPV, with separation of the posterior hyaloid, was performed in 10 eyes with DME and signs of traction. Lewis obtained BCVA gains in 9 eyes, and resolution of traction and edema in 8 of them. These first good results have not been confirmed in later studies, which presented differences in the inclusion criteria, the variables analyzed, and the surgical techniques used. See Figures 21 and 22 for examples of DME before and after surgical intervention.

Presence of Traction. Vitreomacular traction is a relevant factor in the generation and maintenance of DME. Hence, its elimination should result in resolution of edema and BCVA gains. Pendergast analyzed the results of PPV in 55 eyes with DME and vitreoretinal interface changes on funduscopy [110]. Although the edema improved, BCVA gains could only be observed in half of the patients. A later study, Protocol D, designed by the DRCR. net [111], evaluated the impact of PPV on the visual and anatomical results of DME with vitreomacular traction, diagnosed by OCT. This multicenter prospective study included 87 eyes from 87 patients. Epiretinal membrane removal, ILM peeling, and laser therapy were allowed during the surgery, and these techniques were used in 61, 54 , and $40 \%$ of cases, respectively. One year following the intervention, $26 \%$ of eyes needed adjuvant treatment for DME and the mean BCVA improved from 20/100 to $20 / 80$. There was a BCVA gain of $\geq 2$ ETDRS lines in $38 \%$ and a worsening of $\geq 2$ ETDRS lines in $26 \%$. Nevertheless, the mean subfoveal thickness measured by OCT decreased from 491 to $256 \mu \mathrm{m}$.

The presence of vitreoretinal macular traction can generate macular edema per se, and a release of this anterior-posterior vector force is recommended in order to recover the prior anatomical situation. However, surgery is controversial when tangential traction is present, especially with regard to the presence of vascular permeability alteration. In the aforementioned Protocol D study by the DRCR.net, the prognostic factors of final BCVA in 241 eyes that underwent PPV for DME treatment were analyzed [112]. In a multivariate analysis, ILM peeling during the procedure was associated with retinal thinning, and epiretinal membrane removal was associated with BCVA gain. Nonetheless, a correlation of intervention with BCVA was not observed, even when only the pseudophakic group was analyzed. In another study, the role of ILM peeling was analyzed in a group of 58 eyes with DME and "thickened posterior hyaloid membrane," in whom PPV with and without ILM removal was performed [113]. This small study showed a significant BCVA gain in both groups, but ILM peeling offered no advantages related to the observed BCVA change.

Nontractional DME. The results of PPV with or without ILM peeling for DME treatment have shown BCVA gains of $\geq 2$ ETDRS lines in around 50\% (range, 20-90\%)
Schmidt-Erfurth et al. 


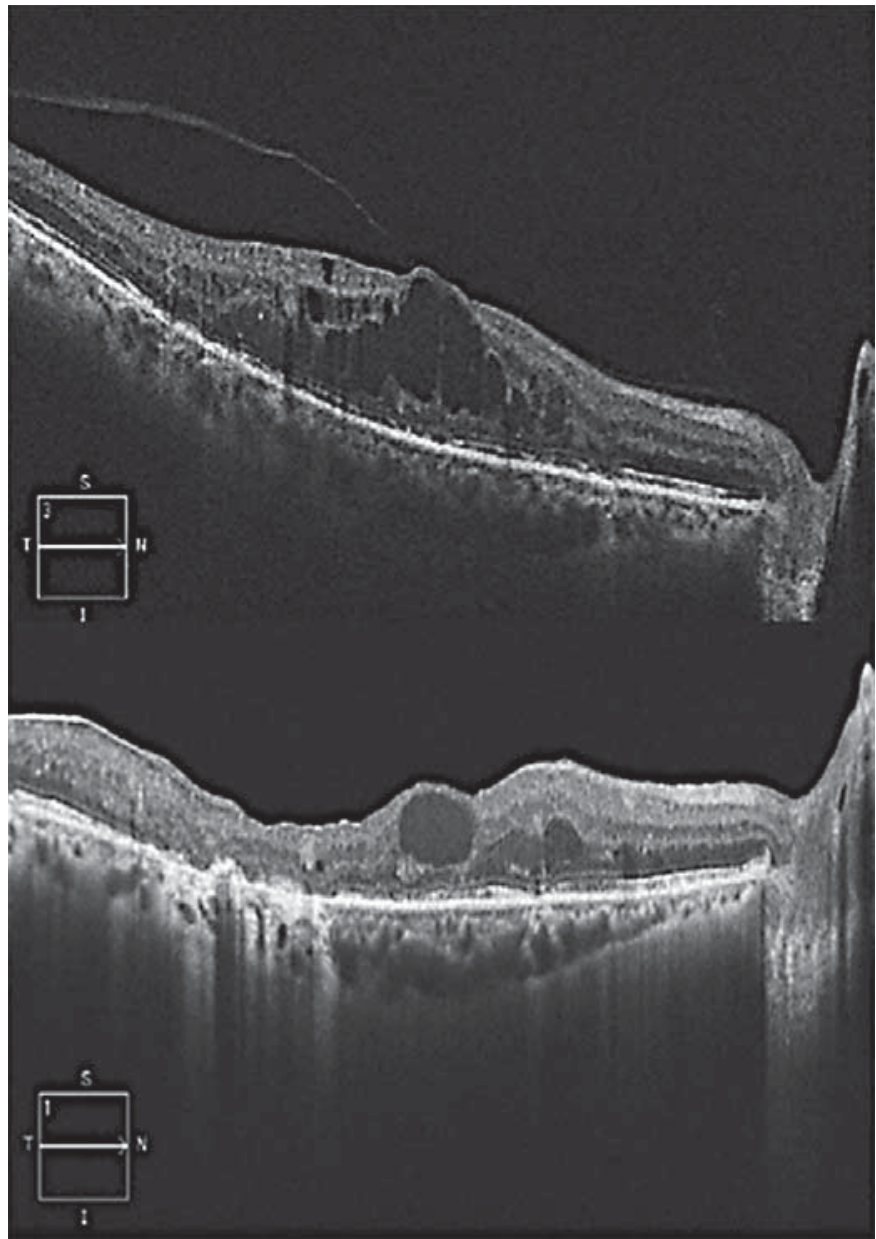

Fig. 22. SD-OCT of a patient with DME unresponsive to laser and intravitreal treatment. Vitreomacular adhesion and macular edema are present (upper image); macular thickness reduction is present after PPV with ILM peeling; however, some intraretinal cystoid fluid remains in the macular region 6 months after the surgery (lower image). Images are courtesy of Pepe Garcia Arumi and Sergio Copete Piqueras.

of cases that could be maintained in the long term [114122]. The wide range observed in BCVA outcomes may be the result of differences in the inclusion criteria, the number of patients and the treatment used. Kumagai et al. [118] retrospectively analyzed the outcome of PPV in 356 eyes over 5 years and reported a mean BCVA change from 0.72 to 0.5 (LogMAR). A BCVA gain of $0.2 \log$ MAR units was observed in $48 \%$ of cases, $39 \%$ did not show changes, and $13 \%$ experienced worsening by $0.2 \log$ MAR units; no correlation between the changes in CRT and BCVA could be observed, although there was a constant decrease in CRT. Yamamoto analyzed progression of

Guidelines for the Management of Diabetic Macular Edema by EURETINA
CRT after PPV and observed a significant reduction as early as 1 week after surgery that continued to gradually decrease at each monthly visit [114]. One should keep in mind, that removal of the vitreous alone which is always combined with membrane peeling improves the oxygenation of the retina.

Stolba et al. [123] compared PPV plus ILM removal with observation in 56 eyes with diffuse DME since 6-18 months; posterior hyaloid attachment was present in all patients and grid laser therapy had been performed at least 4 months before data analysis. BCVA improved in the treated group and deteriorated in the controls. After 6 months, $52 \%$ of eyes undergoing PPV had a BCVA gain of $>10$ letters versus $12.9 \%$ in the control group. Furthermore, a BCVA decrease of $>10$ letters was higher in controls than in the treated patients ( 41.9 vs. $12.9 \%)$. Retinal thickness had decreased 1 month after surgery and remained stable during follow-up, whereas there were no retinal changes in the controls. However, combined PPV and cataract surgery was performed in patients older than 60 years with mild cataract and represents a strong confounding factor. Yanyali et al. [124] recruited 10 patients with bilateral DME unresponsive to grid laser therapy. PPV with ILM peeling was performed in one eye and the fellow was left untreated. The within-group and between-group BCVA changes were not significant, but there was a higher percentage of improvements by $\geq 2$ ETDRS lines in the PPV group (40 vs. 10\%). Moreover, the anatomical analysis showed a larger reduction in foveal thickness in the PPV group (165.8 vs. $37.8 \mu \mathrm{m}, p<$ 0.05).

Comparisons between laser and PPV treatment for DME have yielded controversial results. Yanyali et al. [125] included both eyes of 12 patients without prior laser treatment in another prospective study. In a randomized fashion, one eye underwent vitrectomy and the fellow eye received a single grid laser session. At 6 months, a significant mean BCVA gain was found in the PPV group only (and a BCVA gain of $\geq 2$ ETDRS lines in 50 [PPV] vs. $25 \%$ [laser]). OCT showed a significant improvement in the surgery arm only as well, highlighting the inefficiency of laser rather than a superiority of surgery. Kumar et al. [126] prospectively studied 24 eyes with BCVA $\leq 6 / 60$, unresponsive to prior laser, randomizing patients to PPV with ILM peeling or grid laser. There was no difference in mean BCVA gains in both treatment groups, whereas anatomic evaluation showed that surgery improved mean CRT in the surgery group more, but there was no correlation between CRT and BCVA gain. Thomas et al. [127] recruited 40 eyes with nontractional DME

Ophthalmologica 2017;237:185-222 213 
and prior macular laser treatment, randomizing eyes to PPV or further macular laser. After 1 year, no functional or anatomical advantages were observed in the PPV arm. There were some differences in the mean duration of diabetes, mean hemoglobin $\mathrm{A} 1 \mathrm{c}(\mathrm{HbA1c})$ and the use of insulin between the groups, which may have been confounding factors. Patel et al. [128] randomized 15 eyes to PPV without ILM peeling or to macular laser treatment. Although BCVA after 1 year was slightly better in the laser arm, anatomical and cone function results (measured by fine matrix mapping) were better in the PPV group. All of these interventional studies fail to show anything but the inefficacy of both laser and surgery as compared to anti-VEGF benefits.

Intravitreal injection of triamcinolone acetonide has also been compared with PPV in DME patients. In a prospective study of 40 eyes from 20 patients with bilateral DME, Doi et al. [129] compared PPV treatment in one eye and a single intravitreal injection of $4 \mathrm{mg}$ triamcinolone acetonide in the fellow eye. After 1 year, there was no significant BCVA gain from baseline and no significant difference in BCVA change between the groups. Mean CRT showed differences between the groups: in the triamcinolone arm it decreased in the first month, but afterwards increased to the pretreatment level, while in the surgery arm CRT decreased progressively, being significantly thinner after 1 year. In a retrospective study of 40 eyes treated with a combination of laser, triamcinolone, and PPV, Kim et al. [130] reported BCVA gains and CRT decrease after 3 years, with an increase of $\geq 2$ ETDRS lines in $65 \%$ of cases.

Stefaniotou et al. [131] retrospectively analyzed the results obtained in 73 eyes treated with PPV with or without ILM peeling. BCVA gains could be observed in both groups, but a greater improvement could be seen in patients undergoing ILM peeling. These results are in contrast with those of Hoerauf et al. [132] and Bardak et al. [133] who reported no significantly greater BCVA gain when ILM peeling was added to PPV. The study with the longest follow-up was conducted by Kumagai et al. [134]. These authors analyzed 116 eyes from 58 patients in whom PPV was undertaken in both eyes, with the addition of ILM peeling in one of them only. In both groups, $75 \%$ of eyes showed BCVA gains after 5 years of followup, with no significant differences between the 2 groups. In studies by Figueroa et al. [135] and Shiba et al. [136], no advantages of ILM peeling over conventional or trigon-assisted vitrectomy were reported. All of these studies were small and not stratified regarding their baseline characteristics.
Presence of Traction and Nontractional DME in Comparison. The presence of traction has been considered an indication for surgery, and the results are thought to be better than those of DME without signs of traction. In a prospective study, Shah et al. [137] analyzed factors predicting the outcome of PPV for DME after 1 year and reported that eyes with vitreoretinal traction had higher BCVA gains than nontractional cases. In contrast, the retrospective study by Bonnin et al. [138] of 73 unresponsive eyes with DME with or without traction followed for a mean of 5.3 years reported improvements in BCVA and CRT after 3 years with no significant differences between the 2 groups. The percentage of patients that gained $\geq 2$ ETDRS lines at the final visit was also similar ( 55 vs. $56 \%$ ). Hence, no solid recommendation favoring surgical intervention can be deducted from these studies.

Predictive Factors for Successful Surgical Therapy. Several factors have been associated with the final BCVA following PPV. A poorer preoperative BCVA has been associated with higher BCVA gains [112]. Greater retinal thickness, poorer glycemic control [139], presence of SRF [137], lack of ELM integrity [140] and disruption of the ellipsoid zone [141] have been associated with a poorer final absolute BCVA.

\section{Recommendation}

DME treatment has vastly evolved in the last decade with the development of intravitreal anti-VEGF injection therapy and dexamethasone implants, which has put surgery into a second-line position. DME surgery should be considered based on the vitreous and retinal status. The presence of anterior-posterior traction may be an indication for PPV in eyes with DME. Tangential traction due to an epiretinal or hyaloid membrane should be considered only when the response to anti-VEGF or dexamethasone implants is incomplete. There is no consensus regarding the advantages of PPV when there is no evidence of traction. Nonetheless, PPV has been proven to maintain BCVA, and it should be considered for cases unresponsive to intravitreal treatments and no posterior vitreous detachment, keeping in mind that the anatomical outcome will be better than the functional results. Further studies are needed to evaluate the effectiveness of surgery in eyes that have previously been treated with the drugs available on the market. The recommendation for PPV is given when traction is present based on evidence levels II-III.
214

Ophthalmologica 2017;237:185-222 DOI: $10.1159 / 000458539$
Schmidt-Erfurth et al. 


\section{Systemic Diabetic Disease and Management}

\section{The Role of Metabolic Control}

Rationale

Diabetes mellitus as a systemic disease can have various macrovascular as well as microvascular complications. Among these, DR and particularly DME play a major role as they are the major cause of blindness, and the number of people suffering from diabetes mellitus is increasing worldwide [142-144]. Therefore, the role of metabolic control needs to be evaluated whenever talking about the treatment of DR and particularly DME, a "side effect" of a systemic disease.

\section{Evidence}

The role of metabolic control includes the question about the relevant target levels of glycemia and blood pressure for the prevention of the development or progression of DR, whether some hypoglycemic drugs and blood pressure-lowering agents are more effective than others for the prevention of DR, and the role of lipidlowering agents [145]. The ETDRS study identified important risk factors for progression to high-risk PDR including more severe stages of DR, decreased BCVA or increased DME, younger age or type 1 versus type 2 diabetes mellitus and high levels of HbA1c [146, 147]. The list of risk factors for the progression to PDR also included a low hematocrit and increased serum lipids as surrogates of a low quality of metabolic control. The long-term benefit of improving glycemic control was evaluated by large prospective studies such as the Diabetes Control and Complications Trial (DCCT) and the United Kingdom Prospective Diabetes Study (UKPDS) [84, 148-154]. They provided strong evidence that tight control of glycemia (with a glycosylated hemoglobin [HbAlc] of $7 \%$ or less) reduced the risk of development and progression of DR in both type 1 and type 2 diabetes mellitus.

The DCCT addressed the questions whether in patients with insulin-dependent diabetes mellitus an intensive therapy completely prevented the development of $\mathrm{DR}$, whether the potential effect of therapy was dependent on the preexisting stage of DR, and which other factors, besides the intensity of treatment, influenced the effectiveness of therapy [148, 150-152]. The DCCT included 1,441 patients with insulin-dependent diabetes mellitus. The total study population was divided into a primary prevention cohort with a diabetes duration ranging between 1 and 5 years and without DR at baseline and into a secondary intervention cohort with $1-15$ years' duration and minimal to moderate nonproliferative DR.

Guidelines for the Management of Diabetic Macular Edema by EURETINA
The patients were randomly assigned to either intensive treatment, aiming at achieving glycemic levels as close to the normal range as possible or to conventional diabetes therapy. The DCCT revealed that an intensive treatment reduced the risks of DR, nephropathy, and neuropathy by $35-90 \%$ when compared with the conventional treatment. The risks of DR and nephropathy increased with higher mean HbAlc level and higher body mass index. The earlier an intensive treatment was started, the more effective it was, in particular if complications had not developed yet. The risk reduction was maintained through 7 years of follow-up, although the difference between the former intensive treatment group and the conventional treatment group decreased and eventually became statistically nonsignificant by 5 years. The further rate of progression of complications from their levels at the end of the DCCT remained less in the former intensive treatment group. The conclusions from the DCCT were that the benefits of 6.5 years of intensive treatment extended well beyond the period of its most intensive implementation, that intensive treatment should be started as soon as it is safely possible after the onset of type 1 diabetes mellitus and maintained thereafter and that one should aim for a practicable target $\mathrm{HbA} 1 \mathrm{c}$ level of $7.0 \%$ or less.

The UKPDS addressed the question whether improved blood-glucose control had an effect on macrovascular complications, while it simultaneously decreased the progression of diabetic microvascular disease such as DR [149]. It included more than 3,800 newly diagnosed patients with type 2 diabetes with a median age of 54 years, who showed a mean of 2 fasting plasma glucose concentrations of $6.1-15.0 \mathrm{mmol} / \mathrm{L}$ after initiation of diet of 3 months and who were randomly assigned to (1) an intensive treatment arm with (1a) sulfonylurea or (1b) insulin or to (2) a conventional treatment arm with diet. Over 10 years, $\mathrm{HbAlc}$ was $7.0 \%$ in the intensive care group compared with $7.9 \%$ in the conventional treatment group, with no significant difference in $\mathrm{HbAlc}$ between the 2 subgroups within the intensive care arm. Compared with the conventional group, the risk in the intensive care group was $12 \%$ lower for any diabetes-related endpoint, with the most marked impact with a $25 \%$ risk reduction on the microvascular endpoints, including the need for retinal laser therapy for DR. It was concluded that intensive blood-glucose control by either sulfonylureas or insulin substantially decreased the risk of microvascular complications including DR in patients with type 2 diabetes, while none of the drugs had an adverse effect on cardiovascular outcomes, and while all intensive treatment increased the risk of hypoglycemia [149].

Ophthalmologica 2017;237:185-222

DOI: $10.1159 / 000458539$ 
In a post-trial monitoring of the participants of the UKPDS, 4,209 out of 5,102 patients with newly diagnosed type 2 diabetes were randomly assigned to receive either conventional therapy (dietary restriction) or intensive therapy (either sulfonylurea or insulin or, in overweight patients, metformin) for glucose control [155]. Although differences between the 2 groups in $\mathrm{HbAlc}$ levels were lost after the first year of the study, the intensive care sulfonylurea-insulin group showed a relative reduction in risk also after 10 years for any diabetes-related endpoint $(9 \%, p=0.04)$ and microvascular disease $(24 \%, p=0.001)$. One inferred that despite an early loss of glycemic differences, a continued reduction in microvascular risk and emergent risk reductions for myocardial infarction and death from any cause were observed during 10 years of posttrial follow-up of the UKPDS. Another study of the UKPDS was conducted to examine whether intensive glucose control with metformin had any specific advantage or disadvantage as compared with insulin or sulfonylurea therapy in patients with type 2 diabetes to decrease progression of microvascular disease and to reduce the risk of heart attacks [156]. Among patients allocated to intensive blood-glucose control, metformin showed a greater effect than chlorpropamide, glibenclamide, or insulin for any diabetes-related endpoint $(p=0.003)$ including DR, all-cause mortality $(p=0.02)$, and stroke $(p=0.03)$. The authors concluded that since intensive glucose control with metformin decreased the risk of diabetes-related endpoints in overweight diabetic patients and since it was associated with less weight gain and fewer hypoglycemic attacks than it was with insulin and sulfonylureas, metformin might be the first-line pharmacological therapy of choice in these patients [155].

In the Fenofibrate Intervention and Event Lowering in Diabetes (FIELD) Study, 9,795 patients aged $50-75$ years with type 2 diabetes mellitus were randomly assigned to receive fenofibrate $200 \mathrm{mg} /$ day $(n=4,895)$ or matching placebo $(n=4,900)$ [157]. At each clinic visit, information concerning laser treatment for DR - a prespecified tertiary endpoint of the main study - was gathered. It revealed that laser treatment was needed more frequently in participants with poorer glycemic or blood pressure control than in those with good control of these factors and in those with a greater burden of clinical microvascular disease, but the need for such treatment was not affected by plasma lipid concentrations. The FIELD Study also showed that the requirement for the first laser treatment for any retinopathy was significantly lower in study participants taking fenofibrate than in study participants without fenofibrate therapy.

216

Ophthalmologica 2017;237:185-222 DOI: $10.1159 / 000458539$
Several other smaller-scaled studies also evaluated the relationship between the glycemic control and DR and arrived at similar conclusions $[145,147,154]$. Intensive glycemic control reduced the risk of any retinopathy by approximately $27 \%$. Intensive therapy was most effective when initiated early in the course of diabetes, demonstrating a beneficial effect over the course and progression of retinopathy. The long-term benefits of the intensive glycemic control greatly outweighed the risk of an early worsening of the retinopathy in the early phase of the therapy [158]. Every percent reduction in HbA1c (e.g., from 9 to $8 \%$ ) lowered the risk of retinopathy by $30-40 \%$ and the effect appeared long lasting (metabolic memory) [153]. To avoid any waning of the beneficial effect of intensive care therapy of diabetes mellitus, $\mathrm{HbA} 1 \mathrm{c}$ should be maintained at target values as long as possible [84]. Correspondingly, a meta-analysis of 3 populationbased studies showed a graded relation between the level of glycemia and frequency of DR signs, even below the diagnostic criterion for diabetes (fasting plasma glucose of $7.0 \mathrm{mmol} / \mathrm{L}$ ) [159].

In contrast to the findings obtained in the studies just described, the Action in Diabetes and Vascular Disease (ADVANCE) trial reported that aggressive glycemic control with an $\mathrm{HbA} 1 \mathrm{c}$ of $<6.5 \%$ did not substantially affect the development or progression of DR in patients with type 2 diabetes $[160,161]$. The Action to Control Cardiovascular Risk in Diabetes (ACCORD) trial showed that such aggressive glycemic control could be associated with increased mortality, although the cause of unexpected excess deaths remains unclear [162]. The Veterans Affairs Diabetes Trial revealed no significant benefits of intensive glycemic control with an HbAlc of $<6.9 \%$ on DR outcomes after 5 years of follow-up [163]. The reason for the difference between these studies and the UKPDS could be related to the differences in the study populations, the length of follow-up, the timing of the therapy and the influence of other contributing factors such as the control of arterial hypertension.

Lowering elevated serum lipid levels has been shown to decrease the risk of cardiovascular morbidity. The ETDRS data suggested that lipid lowering may also decrease the risk of hard exudate formation and associated vision loss in patients with DR $[146,147]$. The DCCT study revealed that the severity of DR was correlated with increasing blood concentrations of triglycerides and negatively associated with the concentration of high-density lipoprotein cholesterol [164]. In the FIELD study, fenofibrate, a lipid-modifying drug, reduced the need for laser treatment of vision-threatening DR by $31 \%$ in patients 
with type 2 diabetes $[157,165]$. Interestingly, this observation did not seem to be attributable to measurable changes in blood lipid concentrations, suggesting that other as yet unknown mechanisms could have played a role.

\section{Recommendation}

Clearly, diabetes as a severe and chronic systemic disease must be managed systemically by expert physicians, which is an endocrinologist or an internal medicine specialist. Ophthalmologists who may be the first ones to identify type 2 diabetes by ophthalmoscopy have an important responsibility to instruct the patient about the need to have the diabetic control consolidated. Asking for the $\mathrm{HbA} 1 \mathrm{c}$ levels gives an orientation about the systemic condition and may lead to rereferral to the diabetologist. Another systemic feature worsening DME as well as DRP is systemic hypertension, which also requires a systemic approach. Interestingly, $\mathrm{HbA} 1 \mathrm{c}$ levels do not correspond tightly to anti-VEGF treatment effects in the eye, and neither the absolute benefit nor the prognosis is associated with $\mathrm{HbA1}$ c levels. Timing of ocular therapy on the other hand is very important, suggesting early ocular intervention and systemic workup should not substantially delay retinal therapy. The tightness of glycemic management and ups and downs in this regimen appear to influence the progression of DRP; therefore, a solid communication between the diabetologist and the retinologist is crucial.

\section{Acknowledgements}

We acknowledge the help of Maurizio Battaglia Parodi, Aude Couturier, Roy Schwartz, Sergio Copete Piqueras in preparing the manuscript material.

\section{References}

1 Klein R, Klein BE, Moss SE, Davis MD, DeMets DL: The Wisconsin epidemiologic study of diabetic retinopathy. IV. Diabetic macular edema. Ophthalmology 1984;91: 1464-1474.

2 Kylstra JA, Brown JC, Jaffe GJ, Cox TA, Gallemore R, Greven CM, Hall JG, Eifrig DE: The importance of fluorescein angiography in planning laser treatment of diabetic macular edema. Ophthalmology 1999;106:2068-2073.

3 Bresnick GH: Diabetic macular edema. A review. Ophthalmology 1986;93:989-997.

4 Carpineto P, Mastropasqua R, Marchini G, Toto L, Di Nicola M, Di Antonio L: Reproducibility and repeatability of foveal avascular zone measurements in healthy subjects by optical coherence tomography angiography. $\mathrm{Br}$ J Ophthalmol 2016;100:671-676.

5 Hwang TS, Jia Y, Gao SS, Bailey ST, Lauer AK, Flaxel CJ, Wilson DJ, Huang D: Optical coherence tomography angiography features of diabetic retinopathy. Retina 2015;35:23712376.

6 Hwang TS, Gao SS, Liu L, Lauer AK, Bailey ST, Flaxel CJ, Wilson DJ, Huang D, Jia Y: Automated quantification of capillary nonperfusion using optical coherence tomography angiography in diabetic retinopathy. JAMA Ophthalmol 2016;134:367-373.

7 Shahlaee A, Pefkianaki M, Hsu J, Ho AC: Measurement of foveal avascular zone dimensions and its reliability in healthy eyes using optical coherence tomography angiography. Am J Ophthalmol 2016;161:50-55.e51.
8 Takase N, Nozaki M, Kato A, Ozeki H, Yoshida M, Ogura Y: Enlargement of foveal avascular zone in diabetic eyes evaluated by en face optical coherence tomography angiography. Retina 2015;35:2377-2383.

9 Bradley PD, Sim DA, Keane PA, Cardoso J, Agrawal R, Tufail A, Egan CA: The evaluation of diabetic macular ischemia using optical coherence tomography angiography. Invest Ophthalmol Vis Sci 2016;57:626-631.

10 Couturier A, Mane V, Bonnin S, Erginay A, Massin P, Gaudric A, Tadayoni R: Capillary plexus anomalies in diabetic retinopathy on optical coherence tomography angiography. Retina 2015;35:2384-2391.

11 Early Treatment Diabetic Retinopathy Study Research Group: Treatment techniques and clinical guidelines for photocoagulation of diabetic macular edema. Early Treatment Diabetic Retinopathy Study Report Number 2 . Ophthalmology 1987;94:761-774.

12 Chew EY, Murphy RP, Newsome DA, Fine SL: Parafoveal telangiectasis and diabetic retinopathy. Arch Ophthalmol 1986;104:71-75.

13 Bolz M, Lammer J, Deak G, Pollreisz A, Mitsch C, Scholda C, Kundi M, Schmidt-Erfurth U: SAVE: a grading protocol for clinically significant diabetic macular oedema based on optical coherence tomography and fluorescein angiography. $\mathrm{Br} \mathrm{J}$ Ophthalmol 2014;98:1612-1617.

14 Kozak I, El-Emam SY, Cheng L, Bartsch DU, Chhablani J, Freeman WR, Arevalo JF: Fluorescein angiography versus optical coherence tomography-guided planning for macular laser photocoagulation in diabetic macular edema. Retina 2014;34:1600-1605.
15 Wang H, Chhablani J, Freeman WR, Chan CK, Kozak I, Bartsch DU, Cheng L: Characterization of diabetic microaneurysms by simultaneous fluorescein angiography and spectral-domain optical coherence tomography. Am J Ophthalmol 2012;153:861-867. e861.

16 Hua R, Liu L, Wang X, Chen L: Imaging evidence of diabetic choroidopathy in vivo: angiographic pathoanatomy and choroidal-enhanced depth imaging. PLoS One 2013; 8:e83494.

17 Dmuchowska DA, Krasnicki P, Mariak Z: Can optical coherence tomography replace fluorescein angiography in detection of ischemic diabetic maculopathy? Graefes Arch Clin Exp Ophthalmol 2014;252:731-738.

18 Wessel MM, Nair N, Aaker GD, Ehrlich JR, D’Amico DJ, Kiss S: Peripheral retinal ischaemia, as evaluated by ultra-widefield fluorescein angiography, is associated with diabetic macular oedema. Br J Ophthalmol 2012;96: 694-698.

19 Patel RD, Messner LV, Teitelbaum B, Michel KA, Hariprasad SM: Characterization of ischemic index using ultra-widefield fluorescein angiography in patients with focal and diffuse recalcitrant diabetic macular edema. Am J Ophthalmol 2013;155:1038-1044.e1032.

20 Schmidt-Erfurth U, Chong V, Loewenstein A, Larsen M, Souied E, Schlingemann R, Eldem B, Mones J, Richard G, Bandello F: Guidelines for the management of neovascular age-related macular degeneration by the European Society of Retina Specialists (EURETINA). Br J Ophthalmol 2014;98:1144-1167.
Guidelines for the Management of Diabetic Macular Edema by EURETINA
Ophthalmologica 2017;237:185-222 DOI: $10.1159 / 000458539$ 
21 Gerendas B, Simader C, Deak GG, Prager SG, Lammer J, Waldstein SM, Kundi M, SchmidtErfurth U: Morphological parameters relevant for visual and anatomic outcomes during anti-VEGF therapy of diabetic macular edema in the RESTORE trial. Invest Ophthalmol Vis Sci 2014;55:1791-1791.

22 Bolz M, Kriechbaum K, Simader C, Deak G, Lammer J, Treu C, Scholda C, Prünte C, Schmidt-Erfurth U: In vivo retinal morphology after grid laser treatment in diabetic macular edema. Ophthalmology 2010;117:538544.

23 Nguyen QD, Brown DM, Marcus DM, Boyer DS, Patel S, Feiner L, Gibson A, Sy J, Rundle AC, Hopkins JJ, Rubio RG, Ehrlich JS: Ranibizumab for diabetic macular edema: results from 2 phase III randomized trials: RISE and RIDE. Ophthalmology 2012;119:789-801.

24 Mitchell P, Bandello F, Schmidt-Erfurth U, Lang GE, Massin P, Schlingemann RO, Sutter F, Simader C, Burian G, Gerstner O, Weichselberger A: The RESTORE Study: Ranibizumab Monotherapy or Combined with Laser versus Laser Monotherapy for Diabetic Macular Edema. Ophthalmology 2011;118: 615-625.

25 Lang GE, Berta A, Eldem BM, Simader C, Sharp D, Holz FG, Sutter F, Gerstner O, Mitchell P: Two-year safety and efficacy of ranibizumab $0.5 \mathrm{mg}$ in diabetic macular edema: interim analysis of the RESTORE extension study. Ophthalmology 2013;120:2004-2012.

26 Schmidt-Erfurth U, Lang GE, Holz FG, Schlingemann RO, Lanzetta P, Massin P, Gerstner O, Bouazza AS, Shen H, Osborne A, Mitchell P: Three-year outcomes of individualized ranibizumab treatment in patients with diabetic macular edema: the RESTORE extension study. Ophthalmology 2014;121: 1045-1053.

27 Elman MJ, Aiello LP, Beck RW, Bressler NM, Bressler SB, Edwards AR, Ferris FL, 3rd, Friedman SM, Glassman AR, Miller KM, Scott IU, Stockdale CR, Sun JK: Randomized trial evaluating ranibizumab plus prompt or deferred laser or triamcinolone plus prompt laser for diabetic macular edema. Ophthalmology 2010;117:1064-1077.e1035.

28 Korobelnik JF, Do DV, Schmidt-Erfurth U, Boyer DS, Holz FG, Heier JS, Midena E, Kaiser PK, Terasaki H, Marcus DM, Nguyen QD, Jaffe GJ, Slakter JS, Simader C, Soo Y, Schmelter T, Yancopoulos GD, Stahl N, Vitti R, Berliner AJ, Zeitz O, Metzig C, Brown DM: Intravitreal aflibercept for diabetic macular edema. Ophthalmology 2014;121:2247-2254.

29 Wells JA, Glassman AR, Ayala AR, Jampol LM, Aiello LP, Antoszyk AN, Arnold-Bush B, Baker CW, Bressler NM, Browning DJ, Elman MJ, Ferris FL, Friedman SM, Melia M, Pieramici DJ, Sun JK, Beck RW: Aflibercept, bevacizumab, or ranibizumab for diabetic macular edema. N Engl J Med 2015;372: 1193-1203.
30 Prunte C, Fajnkuchen F, Mahmood S, Ricci F, Hatz K, Studnicka J, Bezlyak V, Parikh S, Stubbings WJ, Wenzel A, Figueira J: Ranibizumab $0.5 \mathrm{mg}$ treat-and-extend regimen for diabetic macular oedema: the RETAIN study. Br J Ophthalmol 2016;100:787-795.

31 Sun JK, Lin MM, Lammer J, et al: Disorganization of the retinal inner layers as a predictor of visual acuity in eyes with center-involved diabetic macular edema. JAMA Ophthalmol 2014;132:1309-1316.

32 Shin HJ, Lee SH, Chung H, Kim HC: Association between photoreceptor integrity and visual outcome in diabetic macular edema. Graefes Arch Clin Exp Ophthalmol 2012;250: 61-70.

33 Bolz M, Schmidt-Erfurth U, Deak G, Mylonas G, Kriechbaum K, Scholda C: Optical coherence tomographic hyperreflective foci: a morphologic sign of lipid extravasation in diabetic macular edema. Ophthalmology 2009;116: 914-920.

34 Gerendas BS, Waldstein SM, Simader C, Deak G, Hajnajeeb B, Zhang L, Bogunovic H, Abramoff MD, Kundi M, Sonka M, SchmidtErfurth U: Three-dimensional automated choroidal volume assessment on standard spectral-domain optical coherence tomography and correlation with the level of diabetic macular edema. Am J Ophthalmol 2014;158: 1039-1048.

35 Early Treatment Diabetic Retinopathy Study research group: Photocoagulation for diabetic macular edema. Early Treatment Diabetic Retinopathy Study report number 1. Arch Ophthalmol 1985;103:1796-1806.

36 Cheema HR, Al Habash A, Al-Askar E: Improvement of visual acuity based on optical coherence tomography patterns following intravitreal bevacizumab treatment in patients with diabetic macular edema. Int J Ophthalmol 2014;7:251-255.

37 Sophie R, Lu N, Campochiaro PA: Predictors of functional and anatomic outcomes in patients with diabetic macular edema treated with ranibizumab. Ophthalmology 2015;122: 1395-1401.

38 Pelosini L, Hull CC, Boyce JF, McHugh D, Stanford MR, Marshall J: Optical coherence tomography may be used to predict visual acuity in patients with macular edema. Invest Ophthalmol Vis Sci 2011;52:2741-2748.

39 Sun JK, Radwan S, Soliman AZ, Lammer J, Lin MM, Prager SG, Silva PS, Aiello LB, Aiello LP: Neural retinal disorganization as a robust marker of visual acuity in current and resolved diabetic macular edema. Diabetes 2015;64:2560-2570.

40 Yiu G, Manjunath V, Chiu SJ, Farsiu S, Mahmoud TH: Effect of anti-vascular endothelial growth factor therapy on choroidal thickness in diabetic macular edema. Am J Ophthalmol 2014;158:745-751.e742.
41 Bonnin S, Tadayoni R, Erginay A, Massin P, Dupas B: Correlation between ganglion cell layer thinning and poor visual function after resolution of diabetic macular edema. Invest Ophthalmol Vis Sci 2015;56:978-982.

42 Hwang DJ, Lee EJ, Lee SY, Park KH, Woo SJ: Effect of diabetic macular edema on peripapillary retinal nerve fiber layer thickness profiles. Invest Ophthalmol Vis Sci 2014;55: 4213-4219.

43 Rayess N, Rahimy E, Ying G-s, Bagheri N, Ho AC, Regillo CD, Vander JF, Hsu J: Baseline choroidal thickness as a predictor for response to anti-vascular endothelial growth factor therapy in diabetic macular edema. Am J Ophthalmol 2015;159:85-91.e83.

44 Pemp B, Deak G, Prager S, Mitsch C, Lammer J, Schmidinger G, Scholda C, Schmidt-Erfurth U, Bolz M: Distribution of intraretinal exudates in diabetic macular edema during anti-vascular endothelial growth factor therapy observed by spectral domain optical coherence tomography and fundus photography. Retina 2014;34:2407-2415.

45 Domalpally A, Ip MS, Ehrlich JS: Effects of intravitreal ranibizumab on retinal hard exudate in diabetic macular edema: findings from the RIDE and RISE phase III clinical trials. Ophthalmology 2015;122:779-786.

46 Mylonas G, Bolz M, Kriechbaum K, Treu C, Deak G, Lammer J, Scholda C, Schmidt-Erfurth U: Retinal architecture recovery after grid photocoagulation in diabetic macular edema observed in vivo by spectral domain optical coherence tomography. Retina 2013; 33:717-725.

47 Elman MJ, Qin H, Aiello LP, Beck RW, Bressler NM, Ferris FL 3rd, Glassman AR, Maturi RK, Melia M: Intravitreal ranibizumab for diabetic macular edema with prompt versus deferred laser treatment: three-year randomized trial results. Ophthalmology 2012;119:2312-2318.

48 Elman MJ, Ayala A, Bressler NM, Browning D, Flaxel CJ, Glassman AR, Jampol LM, Stone TW: Intravitreal ranibizumab for diabetic macular edema with prompt versus deferred laser treatment: 5-year randomized trial results. Ophthalmology 2015;122:375-381.

49 Martin DF, Maguire MG, Fine SL, Ying GS, Jaffe GJ, Grunwald JE, Toth C, Redford M, Ferris FL 3rd: Ranibizumab and bevacizumab for treatment of neovascular age-related macular degeneration: two-year results. Ophthalmology 2012;119:1388-1398.

50 Gerendas BS, Prager SG, Deak GG, Waldstein SM, Lammer J, Simader C, Kundi M, SchmidtErfurth U: Morphological parameters relevant for long-term outcomes during therapy of diabetic macular edema in the RESTORE Extension trial. Invest Ophthalmol Vis Sci 2015;56:4686-4686.

51 Xu X, Lee K, Zhang L, Sonka M, Abramoff M: Stratified sampling voxel classification for segmentation of intraretinal and subretinal fluid in longitudinal clinical OCT data. IEEE Trans Med Imaging, Epub ahead of print. 
52 Schlegl T, Waldstein SM, Vogl WD, SchmidtErfurth U, Langs G: Predicting semantic descriptions from medical images with convolutional neural networks. Inf Process Med Imaging 2015;24:437-448.

53 Early Treatment Diabetic Retinopathy Study Research Group: Focal photocoagulation treatment of diabetic macular edema. Relationship of treatment effect to fluorescein angiographic and other retinal characteristics at baseline: ETDRS report No. 19. Arch Ophthalmol 1995;113:1144-1155.

54 Network DRCR: A randomized trial comparing intravitreal triamcinolone acetonide and focal/grid photocoagulation for diabetic macular edema. Ophthalmology 2008;115:14471459.e1410.

55 Beck RW, Edwards AR, Aiello LP, Bressler NM, Ferris F, Glassman AR, Hartnett E, Ip MS, Kim JE, Kollman C: Three-year followup of a randomized trial comparing focal/grid photocoagulation and intravitreal triamcinolone for diabetic macular edema. Arch Ophthalmol 2009;127:245-251.

56 Luttrull JK, Dorin G: Subthreshold diode micropulse laser photocoagulation (SDM) as invisible retinal phototherapy for diabetic macular edema: a review. Curr Diabetes Rev 2012; 8:274-284.

57 Lavinsky D, Cardillo JA, Melo LA Jr, Dare A, Farah ME, Belfort R Jr: Randomized clinical trial evaluating mETDRS versus normal or high-density micropulse photocoagulation for diabetic macular edema. Invest Ophthalmol Vis Sci 2011;52:4314-4323.

58 Figueira J, Khan J, Nunes S, Sivaprasad S, Rosa A, de Abreu JF, Cunha-Vaz JG, Chong NV: Prospective randomised controlled trial comparing sub-threshold micropulse diode laser photocoagulation and conventional green laser for clinically significant diabetic macular oedema. Br J Ophthalmol 2009;93: 1341-1344.

59 Gross JG, Glassman AR, Jampol LM, Inusah S, Aiello LP, Antoszyk AN, Baker CW, Berger BB, Bressler NM, Browning D, Elman MJ, Ferris FL 3rd, Friedman SM, Marcus DM, Melia M, Stockdale CR, Sun JK, Beck RW: Panretinal photocoagulation vs intravitreous ranibizumab for proliferative diabetic retinopathy: a randomized clinical trial. JAMA 2015;314:2137-2146

60 Cilley JC, Barfi K, Benson AB 3rd, Mulcahy MF: Bevacizumab in the treatment of colorectal cancer. Expert Opin Biol Ther 2007;7:739749.

61 Mould DR, Sweeney KR: The pharmacokinetics and pharmacodynamics of monoclonal antibodies - mechanistic modeling applied to drug development. Curr Opin Drug Discov Devel 2007;10:84-96.

62 Krohne TU, Eter N, Holz FG, Meyer CH: Intraocular pharmacokinetics of bevacizumab after a single intravitreal injection in humans. Am J Ophthalmol 2008;146:508-512.

Guidelines for the Management of Diabetic Macular Edema by EURETINA
63 Matsuyama K, Ogata N, Matsuoka M, Wada M, Takahashi K, Nishimura T: Plasma levels of vascular endothelial growth factor and pigment epithelium-derived factor before and after intravitreal injection of bevacizumab. $\mathrm{Br} \mathrm{J}$ Ophthalmol 2010;94:1215-1218.

64 Zehetner C, Kirchmair R, Huber S, Kralinger MT, Kieselbach GF: Plasma levels of vascular endothelial growth factor before and after intravitreal injection of bevacizumab, ranibizumab and pegaptanib in patients with agerelated macular degeneration, and in patients with diabetic macular oedema. Br J Ophthalmol 2013;97:454-459.

65 Wells JA, Glassman AR, Ayala AR, Jampol LM, Bressler NM, Bressler SB, Brucker AJ, Ferris FL, Hampton GR, Jhaveri C, Melia M, Beck RW: Aflibercept, bevacizumab, or ranibizumab for diabetic macular edema: twoyear results from a comparative effectiveness randomized clinical trial. Ophthalmology 2016;123:1351-1359.

66 Massin P, Bandello F, Garweg JG, Hansen LL, Harding SP, Larsen M, Mitchell P, Sharp D, Wolf-Schnurrbusch UE, Gekkieva M, Weichselberger A, Wolf S: Safety and efficacy of ranibizumab in diabetic macular edema (RESOLVE Study): a 12-month, randomized, controlled, double-masked, multicenter phase II study. Diabetes Care 2010;33:23992405.

67 Elman MJ, Bressler NM, Qin H, Beck RW, Ferris FL 3rd, Friedman SM, Glassman AR, Scott IU, Stockdale CR, Sun JK: Expanded 2-year follow-up of ranibizumab plus prompt or deferred laser or triamcinolone plus prompt laser for diabetic macular edema. Ophthalmology 2011;118:609-614.

68 Do DV, Nguyen QD, Boyer D, Schmidt-Erfurth U, Brown DM, Vitti R, Berliner AJ, Gao B, Zeitz O, Ruckert R, Schmelter T, Sandbrink $R$, Heier JS: One-year outcomes of the da Vinci Study of VEGF Trap-Eye in eyes with diabetic macular edema. Ophthalmology 2012; 119:1658-1665.

69 Brown DM, Nguyen QD, Marcus DM, Boyer DS, Patel S, Feiner L, Schlottmann PG, Rundle AC, Zhang J, Rubio RG, Adamis AP, Ehrlich JS, Hopkins JJ: Long-term outcomes of ranibizumab therapy for diabetic macular edema: the 36-month results from two phase III trials: RISE and RIDE. Ophthalmology 2013;120:2013-2022.

70 Chakravarthy U, Harding SP, Rogers CA, Downes SM, Lotery AJ, Culliford LA, Reeves BC: Alternative treatments to inhibit VEGF in age-related choroidal neovascularisation: 2 -year findings of the IVAN randomised controlled trial. Lancet 2013;382:12581267.

71 Kodjikian L, Souied EH, Mimoun G, MaugetFaysse M, Behar-Cohen F, Decullier E, Huot L, Aulagner G: Ranibizumab versus bevacizumab for neovascular age-related macular degeneration: results from the GEFAL noninferiority randomized trial. Ophthalmology 2013;120:2300-2309.
72 Berg K, Pedersen TR, Sandvik L, Bragadottir $\mathrm{R}$ : Comparison of ranibizumab and bevacizumab for neovascular age-related macular degeneration according to LUCAS treat-andextend protocol. Ophthalmology 2015;122: 146-152.

73 Solomon SD, Lindsley KB, Krzystolik MG, Vedula SS, Hawkins BS: Intravitreal bevacizumab Versus ranibizumab for treatment of neovascular age-related macular degeneration: findings from a Cochrane systematic review. Ophthalmology 2016;123:70-77.e71.

74 Aiello LP, Avery RL, Arrigg PG, Keyt BA, Jampel HD, Shah ST, Pasquale LR, Thieme H, Iwamoto MA, Park JE, et al: Vascular endothelial growth factor in ocular fluid of patients with diabetic retinopathy and other retinal disorders. N Engl J Med 1994;331:1480-1487.

75 Antonetti DA, Barber AJ, Hollinger LA, Wolpert EB, Gardner TW: Vascular endothelial growth factor induces rapid phosphorylation of tight junction proteins occludin and zonula occluden 1. A potential mechanism for vascular permeability in diabetic retinopathy and tumors. J Biol Chem 1999;274:23463-23467.

76 Boyer DS, Nguyen QD, Brown DM, Basu K, Ehrlich JS: Outcomes with as-needed ranibizumab after initial monthly therapy: longterm outcomes of the phase III RIDE and RISE Trials. Ophthalmology 2015;122:25042513.e2501.

77 Chen G, Li W, Tzekov R, Jiang F, Mao S, Tong Y: Ranibizumab monotherapy or combined with laser versus laser monotherapy for diabetic macular edema: a meta-analysis of randomized controlled trials. PLoS One 2014; 9:e115797.

78 Pearce I, Banerjee S, Burton BJ, Chakravarthy U, Downey L, Gale RP, Gibson J, Pagliarini S, Patel J, Sivaprasad S, Andrews C, Brittain C, Warburton J: Ranibizumab $0.5 \mathrm{mg}$ for diabetic macular edema with bimonthly monitoring after a phase of initial treatment: 18-month, multicenter, phase IIIB RELIGHT study. Ophthalmology 2015;122:1811-1819.

79 Brown DM, Schmidt-Erfurth U, Do DV, Holz FG, Boyer DS, Midena E, Heier JS, Terasaki H, Kaiser PK, Marcus DM, Nguyen QD, Jaffe GJ, Slakter JS, Simader C, Soo Y, Schmelter T, Yancopoulos GD, Stahl N, Vitti R, Berliner AJ, Zeitz O, Metzig C, Korobelnik JF: Intravitreal aflibercept for diabetic macular edema: 100week results from the VISTA and VIVID studies. Ophthalmology 2015;122:2044-2052.

80 Tan CS, Li KZ, Lim TH: Calculating the predicted retinal thickness from spectral domain and time domain optical coherence tomography - comparison of different methods. Graefes Arch Clin Exp Ophthalmol 2014;252: 1491-1499.

81 Leal EC, Manivannan A, Hosoya K, Terasaki T, Cunha-Vaz J, Ambrosio AF, Forrester JV: Inducible nitric oxide synthase isoform is a key mediator of leukostasis and blood-retinal barrier breakdown in diabetic retinopathy. Invest Ophthalmol Vis Sci 2007;48:52575265 . 
82 Zhang X, Zeng H, Bao S, Wang N, Gillies MC: Diabetic macular edema: new concepts in patho-physiology and treatment. Cell Biosci 2014;4:27.

83 Adamis AP, Berman AJ: Immunological mechanisms in the pathogenesis of diabetic retinopathy. Semin Immunopathol 2008;30: 65-84.

84 White NH, Sun W, Cleary PA, Tamborlane WV, Danis RP, Hainsworth DP, Davis MD: Effect of prior intensive therapy in type 1 diabetes on 10-year progression of retinopathy in the DCCT/EDIC: comparison of adults and adolescents. Diabetes 2010;59:1244-1253.

85 Gonzalez-Mariscal L, Betanzos A, Nava P, Jaramillo BE: Tight junction proteins. Prog Biophys Mol Biol 2003;81:1-44.

86 Stewart MW: Corticosteroid use for diabetic macular edema: old fad or new trend? Curr Diab Rep 2012;12:364-375.

87 Tamura H, Miyamoto K, Kiryu J, Miyahara S, Katsuta H, Hirose F, Musashi K, Yoshimura $\mathrm{N}$ : Intravitreal injection of corticosteroid attenuates leukostasis and vascular leakage in experimental diabetic retina. Invest Ophthalmol Vis Sci 2005; 46:1440-1444.

88 Sohn HJ, Han DH, Kim IT, Oh IK, Kim KH, Lee DY, Nam DH: Changes in aqueous concentrations of various cytokines after intravitreal triamcinolone versus bevacizumab for diabetic macular edema. Am J Ophthalmol 2011;152:686-694.

89 Scott IU, Edwards AR, Beck RW, Bressler NM, Chan CK, Elman MJ, Friedman SM, Greven CM, Maturi RK, Pieramici DJ, Shami M, Singerman LJ, Stockdale CR: A phase II randomized clinical trial of intravitreal bevacizumab for diabetic macular edema. Ophthalmology 2007;114:1860-1867.

90 Ip MS, Bressler SB, Antoszyk AN, Flaxel CJ, Kim JE, Friedman SM, Qin H: A randomized trial comparing intravitreal triamcinolone and focal/grid photocoagulation for diabetic macular edema: baseline features. Retina 2008;28:919-930.

91 Callanan DG, Gupta S, Boyer DS, Ciulla TA, Singer MA, Kuppermann BD, Liu CC, Li XY, Hollander DA, Schiffman RM, Whitcup SM: Dexamethasone intravitreal implant in combination with laser photocoagulation for the treatment of diffuse diabetic macular edema. Ophthalmology 2013;120:1843-1851.

92 Boyer DS, Yoon YH, Belfort R Jr, Bandello F, Maturi RK, Augustin AJ, Li XY, Cui H, Hashad Y, Whitcup SM: Three-year, randomized, sham-controlled trial of dexamethasone intravitreal implant in patients with diabetic macular edema. Ophthalmology 2014;121: 1904-1914.

93 Boyer DS, Faber D, Gupta S, Patel SS, Tabandeh H, Li XY, Liu CC, Lou J, Whitcup SM: Dexamethasone intravitreal implant for treatment of diabetic macular edema in vitrectomized patients. Retina 2011;31:915-923.
94 Chang-Lin JE, Attar M, Acheampong AA, Robinson MR, Whitcup SM, Kuppermann $\mathrm{BD}$, Welty D: Pharmacokinetics and pharmacodynamics of a sustained-release dexamethasone intravitreal implant. Invest Ophthalmol Vis Sci 2011;52:80-86.

95 Lam WC, Albiani DA, Yoganathan P, Chen JC, Kherani A, Maberley DA, Oliver A, Rabinovitch T, Sheidow TG, Tourville E, Wittenberg LA, Sigouin C, Baptiste DC: Real-world assessment of intravitreal dexamethasone implant $(0.7 \mathrm{mg})$ in patients with macular edema: the CHROME study. Clin Ophthalmol 2015;9:1255-1268.

96 Campochiaro PA, Brown DM, Pearson A, Ciulla T, Boyer D, Holz FG, Tolentino M, Gupta A, Duarte L, Madreperla S, Gonder J, Kapik B, Billman K, Kane FE: Long-term benefit of sustained-delivery fluocinolone acetonide vitreous inserts for diabetic macular edema. Ophthalmology 2011;118:626635.e622.

97 Cunha-Vaz J, Ashton P, Iezzi R, Campochiaro P, Dugel PU, Holz FG, Weber M, Danis RP, Kuppermann BD, Bailey C, Billman K, Kapik B, Kane F, Green K: Sustained delivery fluocinolone acetonide vitreous implants: long-term benefit in patients with chronic diabetic macular edema. Ophthalmology 2014;121:1892-1903.

98 Campochiaro PA, Hafiz G, Shah SM, Bloom S, Brown DM, Busquets M, Ciulla T, Feiner L, Sabates N, Billman K, Kapik B, Green K, Kane F: Sustained ocular delivery of fluocinolone acetonide by an intravitreal insert. Ophthalmology 2010;117:1393-1399.e1393.

99 Avery RL, Gordon GM: Systemic safety of prolonged monthly anti-vascular endothelial growth factor therapy for diabetic macular edema: a systematic review and metaanalysis. JAMA Ophthalmol 2016;134:2129.

100 Kaiser PK, Riemann CD, Sears JE, Lewis H: Macular traction detachment and diabetic macular edema associated with posterior hyaloidal traction. Am J Ophthalmol 2001;131: 44-49.

101 Nasrallah FP, Jalkh AE, Van Coppenolle F, Kado M, Trempe CL, McMeel JW, Schepens CL: The role of the vitreous in diabetic macular edema. Ophthalmology 1988;95:13351339.

102 Lopes de Faria JM, Jalkh AE, Trempe CL, McMeel JW: Diabetic macular edema: risk factors and concomitants. Acta Ophthalmol Scand 1999;77:170-175.

103 Hikichi T, Fujio N, Akiba J, Azuma Y, Takahashi M, Yoshida A: Association between the short-term natural history of diabetic macular edema and the vitreomacular relationship in type II diabetes mellitus. Ophthalmology 1997;104:473-478.

104 Bhagat N, Grigorian RA, Tutela A, Zarbin MA: Diabetic macular edema: pathogenesis and treatment. Surv Ophthalmol 2009;54: 1-32.
105 Kadonosono K, Itoh N, Ohno S: Perifoveal microcirculation before and after vitrectomy for diabetic cystoid macular edema. Am J Ophthalmol 2000;130:740-744.

106 Park JH, Woo SJ, Ha YJ, Yu HG: Effect of vitrectomy on macular microcirculation in patients with diffuse diabetic macular edema. Graefes Arch Clin Exp Ophthalmol 2009;247:1009-1017.

107 Sebag J, Balazs EA: Pathogenesis of cystoid macular edema: an anatomic consideration of vitreoretinal adhesions. Surv Ophthalmol 1984;28(suppl):493-498.

108 Tamura K, Yokoyama T, Ebihara N, Murakami A: Histopathologic analysis of the internal limiting membrane surgically peeled from eyes with diffuse diabetic macular edema. Jpn J Ophthalmol 2012;56:280-287.

109 Lewis H, Abrams GW, Blumenkranz MS, Campo RV: Vitrectomy for diabetic macular traction and edema associated with posterior hyaloidal traction. Ophthalmology 1992; 99:753-759.

110 Pendergast SD, Hassan TS, Williams GA, Cox MS, Margherio RR, Ferrone PJ, Garretson BR, Trese MT: Vitrectomy for diffuse diabetic macular edema associated with a taut premacular posterior hyaloid. Am J Ophthalmol 2000;130:178-186.

111 Haller JA, Qin H, Apte RS, Beck RR, Bressler NM, Browning DJ, Danis RP, Glassman AR, Googe JM, Kollman C, Lauer AK, Peters MA, Stockman ME: Vitrectomy outcomes in eyes with diabetic macular edema and vitreomacular traction. Ophthalmology 2010; 117:1087-1093.e1083.

112 Flaxel CJ, Edwards AR, Aiello LP, Arrigg PG, Beck RW, Bressler NM, Bressler SB, Ferris FL 3rd, Gupta SK, Haller JA, Lazarus HS, Qin H: Factors associated with visual acuity outcomes after vitrectomy for diabetic macular edema: diabetic retinopathy clinical research network. Retina 2010;30:1488-1495.

113 Bahadir M, Ertan A, Mertoglu O: Visual acuity comparison of vitrectomy with and without internal limiting membrane removal in the treatment of diabetic macular edema. Int Ophthalmol 2005;26:3-8.

114 Yamamoto T, Hitani K, Tsukahara I, Yamamoto S, Kawasaki R, Yamashita H, Takeuchi S: Early postoperative retinal thickness changes and complications after vitrectomy for diabetic macular edema. Am J Ophthalmol 2003;135:14-19.

115 Ikeda T, Sato K, Katano T, Hayashi Y: Improved visual acuity following pars plana vitrectomy for diabetic cystoid macular edema and detached posterior hyaloid. Retina 2000;20:220-222.

116 Dillinger P, Mester U: Vitrectomy with removal of the internal limiting membrane in chronic diabetic macular oedema. Graefes Arch Clin Exp Ophthalmol 2004;242:630637. 
117 Gandorfer A, Messmer EM, Ulbig MW, Kampik A: Resolution of diabetic macular edema after surgical removal of the posterior hyaloid and the inner limiting membrane. Retina 2000;20:126-133.

118 Kumagai K, Furukawa M, Ogino N, Larson E, Iwaki M, Tachi N: Long-term follow-up of vitrectomy for diffuse nontractional diabetic macular edema. Retina 2009;29:464472.

119 La Heij EC, Hendrikse F, Kessels AG, Derhaag PJ: Vitrectomy results in diabetic macular oedema without evident vitreomacular traction. Graefes Arch Clin Exp Ophthalmol 2001;239:264-270.

120 Otani T, Kishi S: A controlled study of vitrectomy for diabetic macular edema. Am J Ophthalmol 2002;134:214-219.

121 Tachi N, Ogino N: Vitrectomy for diffuse macular edema in cases of diabetic retinopathy. Am J Ophthalmol 1996;122:258-260.

122 Yanyali A, Horozoglu F, Celik E, Nohutcu AF: Long-term outcomes of pars plana vitrectomy with internal limiting membrane removal in diabetic macular edema. Retina 2007;27:557-566.

123 Stolba U, Binder S, Gruber D, Krebs I, Aggermann $T$, Neumaier B: Vitrectomy for persistent diffuse diabetic macular edema. Am J Ophthalmol 2005;140:295-301.

124 Yanyali A, Horozoglu F, Celik E, Ercalik Y, Nohutcu AF: Pars plana vitrectomy and removal of the internal limiting membrane in diabetic macular edema unresponsive to grid laser photocoagulation. Eur J Ophthalmol 2006;16:573-581.

125 Yanyali A, Nohutcu AF, Horozoglu F, Celik E: Modified grid laser photocoagulation versus pars plana vitrectomy with internal limiting membrane removal in diabetic macular edema. Am J Ophthalmol 2005;139:795801.

126 Kumar A, Sinha S, Azad R, Sharma YR, Vohra R: Comparative evaluation of vitrectomy and dye-enhanced ILM peel with grid laser in diffuse diabetic macular edema. Graefes Arch Clin Exp Ophthalmol 2007; 245:360-368

127 Thomas D, Bunce C, Moorman C, Laidlaw DA: A randomised controlled feasibility trial of vitrectomy versus laser for diabetic macular oedema. Br J Ophthalmol 2005;89: $81-86$.

128 Patel JI, Hykin PG, Schadt M, Luong V, Bunce C, Fitzke F, Gregor ZJ: Diabetic macular oedema: pilot randomised trial of pars plana vitrectomy vs macular argon photocoagulation. Eye (Lond) 2006;20:873-881.

129 Doi N, Sakamoto T, Sonoda Y, Yasuda M, Yonemoto K, Arimura N, Uchino E, Ishibashi T: Comparative study of vitrectomy versus intravitreous triamcinolone for diabetic macular edema on randomized pairedeyes. Graefes Arch Clin Exp Ophthalmol 2012;250:71-78.
130 Kim YT, Kang SW, Kim SJ, Kim SM, Chung SE: Combination of vitrectomy, IVTA, and laser photocoagulation for diabetic macular edema unresponsive to prior treatments; 3-year results. Graefes Arch Clin Exp Ophthalmol 2012;250:679-684.

131 Stefaniotou M, Aspiotis M, Kalogeropoulos C, Christodoulou A, Psylla M, Ioachim E, Alamanos I, Psilas K: Vitrectomy results for diffuse diabetic macular edema with and without inner limiting membrane removal. Eur J Ophthalmol 2004;14:137-143.

132 Hoerauf $H$, Bruggemann A, Muecke M, Luke J, Muller M, Stefansson E, Hammes HP, Weiss C: Pars plana vitrectomy for diabetic macular edema. Internal limiting membrane delamination vs posterior hyaloid removal. A prospective randomized trial. Graefes Arch Clin Exp Ophthalmol 2011; 249:997-1008.

133 Bardak Y, Cekic O, Tig SU: Comparison of ICG-assisted ILM peeling and triamcinolone-assisted posterior vitreous removal in diffuse diabetic macular oedema. Eye (Lond) 2006;20:1357-1359.

134 Kumagai K, Hangai M, Ogino N, Larson E: Effect of internal limiting membrane peeling on long-term visual outcomes for diabetic macular edema. Retina 2015;35:14221428.

135 Figueroa MS, Contreras I, Noval S: Surgical and anatomical outcomes of pars plana vitrectomy for diffuse nontractional diabetic macular edema. Retina 2008;28:420-426.

136 Shiba T, Kamura Y, Yagi F, Sato Y: Comparison of surgical procedures for vitreous surgery in diabetic macular edema. Jpn J Ophthalmol 2009;53:120-124.

137 Shah SP, Patel M, Thomas D, Aldington S, Laidlaw DA: Factors predicting outcome of vitrectomy for diabetic macular oedema: results of a prospective study. Br J Ophthalmol 2006;90:33-36.

138 Bonnin S, Sandali O, Bonnel S, Monin C, El Sanharawi M: Vitrectomy with internal limiting membrane peeling for tractional and nontractional diabetic macular edema: Long-term Results of a Comparative Study. Retina 2015;35:921-928.

139 Yamada Y, Suzuma K, Ryu M, Tsuiki E, Fujikawa A, Kitaoka T: Systemic factors influence the prognosis of diabetic macular edema after pars plana vitrectomy with internal limiting membrane peeling. Curr Eye Res 2013;38:1261-1265.

140 Chhablani JK, Kim JS, Cheng L, Kozak I, Freeman W: External limiting membrane as a predictor of visual improvement in diabetic macular edema after pars plana vitrectomy. Graefes Arch Clin Exp Ophthalmol 2012;250:1415-1420.

141 Murakami T, Nishijima K, Akagi T, Uji A, Horii T, Ueda-Arakawa N, Muraoka Y, Yoshimura N: Segmentational analysis of retinal thickness after vitrectomy in diabetic macular edema. Invest Ophthalmol Vis Sci 2012;53:6668-6674.
142 Litwak L, Goh SY, Hussein Z, Malek R, Prusty V, Khamseh ME: Prevalence of diabetes complications in people with type 2 diabetes mellitus and its association with baseline characteristics in the multinational Alchieve study. Diabetol Metab Syndr 2013; 5:57.

143 Zimmet P, Alberti KG, Magliano DJ, Bennett PH: Diabetes mellitus statistics on prevalence and mortality: facts and fallacies. Nat Rev Endocrinol 2016;12:616-622.

144 Zimmet PZ, Magliano DJ, Herman WH, Shaw JE: Diabetes: a 21st century challenge. Lancet Diabetes Endocrinol 2014;2:56-64.

145 Cheung N, Mitchell P, Wong TY: Diabetic retinopathy. Lancet 2010;376:124-136.

146 Davis MD, Fisher MR, Gangnon RE, Barton F, Aiello LM, Chew EY, Ferris FL 3rd, Knatterud GL: Risk factors for high-risk proliferative diabetic retinopathy and severe visual loss: Early Treatment Diabetic Retinopathy Study Report \#18. Invest Ophthalmol Vis Sci 1998;39:233-252.

147 Rodriguez-Fontal M, Kerrison JB, Alfaro DV, Jablon EP: Metabolic control and diabetic retinopathy. Curr Diabetes Rev 2009;5: 3-7.

148 Progression of retinopathy with intensive versus conventional treatment in the Diabetes Control and Complications Trial. Diabetes Control and Complications Trial Research Group. Ophthalmology 1995;102: 647-661.

149 Intensive blood-glucose control with sulphonylureas or insulin compared with conventional treatment and risk of complications in patients with type 2 diabetes (UKPDS 33). UK Prospective Diabetes Study (UKPDS) Group. Lancet 1998;352:837-853.

150 Retinopathy and nephropathy in patients with type 1 diabetes four years after a trial of intensive therapy. The Diabetes Control and Complications Trial/Epidemiology of Diabetes Interventions and Complications Research Group. N Engl J Med 2000;342:381389.

151 Zhang L, Krzentowski G, Albert A, Lefebvre PJ: Risk of developing retinopathy in Diabetes Control and Complications Trial type 1 diabetic patients with good or poor metabolic control. Diabetes Care 2001;24:12751279.

152 Writing Team for the Diabetes Control and Complications Trial/Epidemiology of Diabetes Interventions and Complications Research Group: Effect of intensive therapy on the microvascular complications of type 1 diabetes mellitus. JAMA 2002;287:25632569.

153 White NH, Sun W, Cleary PA, Danis RP, Davis MD, Hainsworth DP, Hubbard LD, Lachin JM, Nathan DM: Prolonged effect of intensive therapy on the risk of retinopathy complications in patients with type 1 diabetes mellitus: 10 years after the Diabetes Control and Complications Trial. Arch Ophthalmol 2008;126:1707-1715.
Guidelines for the Management of Diabetic Macular Edema by EURETINA
Ophthalmologica 2017;237:185-222

DOI: $10.1159 / 000458539$ 
154 Fullerton B, Jeitler K, Seitz M, Horvath K, Berghold A, Siebenhofer A: Intensive glucose control versus conventional glucose control for type 1 diabetes mellitus. Cochrane Database Syst Rev 2014:CD009122.

155 Holman RR, Paul SK, Bethel MA, Matthews DR, Neil HA: 10-year follow-up of intensive glucose control in type 2 diabetes. N Engl J Med 2008;359:1577-1589.

156 Effect of intensive blood-glucose control with metformin on complications in overweight patients with type 2 diabetes (UKPDS 34). UK Prospective Diabetes Study (UKPDS) Group. Lancet 1998;352:854-865.

157 Keech AC, Mitchell P, Summanen PA, O’Day J, Davis TM, Moffitt MS, Taskinen MR, Simes RJ, Tse D, Williamson E, Merrifield A, Laatikainen LT, d'Emden MC, Crimet DC, O'Connell RL, Colman PG: Effect of fenofibrate on the need for laser treatment for diabetic retinopathy (FIELD study): a randomised controlled trial. Lancet 2007; 370:1687-1697.

158 Early worsening of diabetic retinopathy in the Diabetes Control and Complications Trial. Arch Ophthalmol 1998;116:874-886.

159 Wong TY, Liew G, Tapp RJ, Schmidt MI, Wang JJ, Mitchell P, Klein R, Klein BE, Zimmet $\mathrm{P}$, Shaw J: Relation between fasting glucose and retinopathy for diagnosis of diabetes: three population-based cross-sectional studies. Lancet 2008;371:736-743.
160 Patel A, MacMahon S, Chalmers J, Neal B, Billot L, Woodward M, Marre M, Cooper M, Glasziou P, Grobbee D, Hamet P, Harrap S, Heller S, Liu L, Mancia G, Mogensen CE, Pan C, Poulter N, Rodgers A, Williams B, Bompoint S, de Galan BE, Joshi R, Travert F: Intensive blood glucose control and vascular outcomes in patients with type 2 diabetes. $\mathrm{N}$ Engl J Med 2008;358:2560-2572.

161 Gerstein HC, Miller ME, Byington RP, Goff DC Jr, Bigger JT, Buse JB, Cushman WC, Genuth S, Ismail-Beigi F, Grimm RH Jr, Probstfield JL, Simons-Morton DG, Friedewald WT: Effects of intensive glucose lowering in type 2 diabetes. N Engl J Med 2008; 358:2545-2559.

162 Liew G, Mitchell P, Wong TY: Systemic management of diabetic retinopathy. BMJ 2009;338:b441.

163 Duckworth W, Abraira C, Moritz T, Reda D, Emanuele N, Reaven PD, Zieve FJ, Marks J, Davis SN, Hayward R, Warren SR, Goldman S, McCarren M, Vitek ME, Henderson WG, Huang GD: Glucose control and vascular complications in veterans with type 2 diabetes. N Engl J Med 2009;360:129-139.
164 Lyons TJ, Jenkins AJ, Zheng D, Lackland DT, McGee D, Garvey WT, Klein RL: Diabetic retinopathy and serum lipoprotein subclasses in the DCCT/EDIC cohort. Invest Ophthalmol Vis Sci 2004;45:910-918.

165 Cheung N, Wong TY: Fenofibrate and diabetic retinopathy. Lancet 2008;371:721-722; author reply 722 .

166 Diabetic retinopathy study. Report Number 6. Design, methods, and baseline results. Report Number 7. A modification of the Airlie House classification of diabetic retinopathy. Prepared by the Diabetic Retinopathy. Invest Ophthalmol Vis Sci 1981;21:1-226.

167 Heng LZ, Pefianaki M, Hykin P, Patel PJ: Interobserver agreement in detecting spectraldomain optical coherence tomography features of diabetic macular edema. PLoS One 2015;10:e0126557.

168 Arevalo JF, Lasave AF, Arias JD, Serrano MA, Arevalo FA: Clinical applications of optical coherence tomography in the posterior pole: the 2011 José Manuel Espino Lecture - Part II. Clin Ophthalmol 2013;7: 2181-2206.

169 Lee J, Rosen R: Optical coherence tomography angiography in diabetes. Curr Diabetes Rep 2016;16:123.

170 Lee J, Moon BG, Cho AR, Yoon YH: Optical coherence tomography angiography of DME and its association with anti-VEGF treatment response. Ophthalmology 2016; $123: 2368-2375$ 